\title{
Fundamentals of direct limit Lie theory
}

\author{
Helge Glöckner
}

\begin{abstract}
We show that every countable direct system of finite-dimensional real or complex Lie groups has a direct limit in the category of Lie groups modelled on locally convex spaces. This enables us to push all basic constructions of finite-dimensional Lie theory to the case of direct limit groups. In particular, we obtain an analogue of Lie's third theorem: every countable-dimensional locally finite real or complex Lie algebra arises as the Lie algebra of some regular Lie group (a suitable direct limit group).
\end{abstract}

\section{Introduction}

In this article, we develop the foundations of Lie theory for countable direct limits of finitedimensional Lie groups. For the purposes of this introduction, consider an ascending sequence $G_{1} \subseteq G_{2} \subseteq \cdots$ of finite-dimensional real Lie groups, such that the inclusion maps are smooth homomorphisms. Then $G:=\bigcup_{n \in \mathbb{N}} G_{n}$ is a group in a natural way, and it becomes a topological group when equipped with the final topology with respect to the inclusion maps $G_{n} \rightarrow G$ (see [HST01, TSH98]). A simple example is $\mathrm{GL}_{\infty}(\mathbb{R})$, the group of invertible matrices of countable size, differing from the unit matrix at only finitely many places. Here $\mathrm{GL}_{\infty}(\mathbb{R})=\bigcup_{n} \mathrm{GL}_{n}(\mathbb{R})$, where $\mathrm{GL}_{1}(\mathbb{R}) \subseteq \mathrm{GL}_{2}(\mathbb{R}) \subseteq \cdots$ identifying $A \in \mathrm{GL}_{n}(\mathbb{R})$ with $\operatorname{diag}(A, 1) \in \mathrm{GL}_{n+1}(\mathbb{R})$. Our goal is to make $G=\bigcup_{n} G_{n}$ a (usually infinite-dimensional) Lie group, and to discuss the fundamental constructions of Lie theory for such groups.

\section{Existing methods}

Provided that certain technical conditions are satisfied (ensuring, in particular, that $\exp _{G}:=$ $\longrightarrow \lim _{G_{n}}: \lim L\left(G_{n}\right) \rightarrow \underline{\lim } G_{n}=G$ is a local homeomorphism at 0 ), the map $\exp _{G}$ restricts

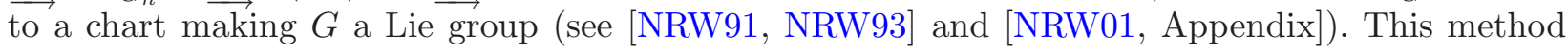
applies, in particular, to $\mathrm{GL}_{\infty}(\mathbb{R})$ and other direct limits of linear Lie groups. It produces Lie groups which are not only smooth, but real analytic in the sense of convenient differential calculus [Glo03a, Remark 6.5]. It is also known that every Lie subalgebra of $\mathfrak{g l}_{\infty}(\mathbb{R}):=\lim _{\longrightarrow} \mathfrak{g l}_{n}(\mathbb{R})$ integrates to a subgroup of $\mathrm{GL}_{\infty}(\mathbb{R})$ (see [KM97, Theorem 47.9]); this provides an alternative construction of the Lie group structure on various direct limit groups. However, neither of these methods is general enough to tackle arbitrary direct limits of Lie groups. In particular, examples show that $\exp _{G}$ need not be injective on any zero-neighbourhood [Glo03a, Example 5.5]. Therefore, a general construction of a Lie group structure on $G=\bigcup_{n} G_{n}$ cannot make use of $\exp _{G}$.

Received 1 August 2004, accepted in final form 1 October 2004.

2000 Mathematics Subject Classification 22E65 (primary); 26E15, 26E20, 26E30, 46T05 (secondary).

Keywords: direct limit, inductive limit, infinite-dimensional Lie group, locally finite Lie algebra, enlargeability, integration of Lie algebras, regular Lie group, universal complexification, convenient differential calculus, homogeneous space, extension of charts, principal bundle.

This journal is (c) Foundation Compositio Mathematica 2005. 


\section{H. GLÖCKNER}

\section{A general construction principle}

In [Glo03a], a smooth Lie group structure on $G=\bigcup_{n} G_{n}$ was constructed in the case where all inclusion maps are embeddings (for 'strict' direct systems). Strict direct limits of Lie groups are discussed there as special cases of direct limits of direct sequences $M_{1} \subseteq M_{2} \subseteq \cdots$ of finite-dimensional smooth manifolds and embeddings onto closed submanifolds. To make $M:=\bigcup_{n} M_{n}$ a smooth manifold, one starts with a chart $\phi_{n_{0}}$ of some $M_{n_{0}}$ and then uses tubular neighbourhoods to extend $\phi_{n}$ already constructed (possibly restricted to a smaller open set) to a chart $\phi_{n+1}$ of $M_{n+1}$. Then $\lim _{\longrightarrow} \phi_{n}$ is a chart for $M$. In the present article, we generalize this construction principle in two ways. First, we are able to remove the strictness condition. This facilitates to make $\bigcup_{n} M_{n}$ a smoothly paracompact, smooth manifold, for any ascending sequence of paracompact, finite-dimensional smooth manifolds and injective immersions (Theorem 3.1, Proposition 3.6). Second, we generalize the method from the case of smooth manifolds over $\mathbb{R}$ to the case of real- and complex analytic manifolds (Theorem 3.1, Proposition 3.8). This enables us to turn $G:=\bigcup_{n} G_{n}$ into a real analytic Lie group in the sense of convenient differential calculus, respectively a complex Lie group, for any ascending sequence of finite-dimensional real or complex Lie groups (Theorem 4.3). ${ }^{1}$ Each direct limit group $G$ is regular in the convenient sense (the argument from [KM97, Theorem 47.8] carries over). Moreover, $G$ is a regular Lie group in Milnor's sense (Theorem 8.1): this is much harder to prove.

\section{Lie theory for direct limit groups}

Despite the fact that $\exp _{G}$ need not be well-behaved, all of the basic constructions of finitedimensional Lie theory can be pushed to the case of direct limit groups $G=\bigcup_{n} G_{n}$. Thus, subgroups and Hausdorff quotient groups are Lie groups (Propositions 7.2 and 7.5), a universal complexification $G_{\mathbb{C}}$ exists (Proposition 7.13), subalgebras of $L(G)$ integrate to analytic subgroups (Proposition 7.11), and Lie algebra homomorphisms integrate to group homomorphisms in the expected way (Proposition 7.10). Furthermore (Theorem 5.1), every locally finite real or complex Lie algebra of countable dimension is enlargeable, i.e. it arises as the Lie algebra of some Lie group (a suitable direct limit group). Such Lie algebras have been studied by Yu. Bahturin, A. A. Baranov, I. Dimitrov, K.-H. Neeb, I. Penkov, H. Strade, N. Stumme, A. E. Zalesskii, and others. If $H \subseteq G$ is a closed subgroup, then $H$ is a conveniently real analytic $\left(c_{\mathbb{R}^{-}}^{\omega}\right)$ submanifold of $G$. Furthermore, the homogeneous space $G / H$ can be given a $c_{\mathbb{R}}^{\omega}$-manifold structure making $\pi: G \rightarrow G / H$ a $c_{\mathbb{R}}^{\omega}$-principal bundle (Proposition 7.5). Similar results are available for complex Lie groups. We remark that special cases of complexifications and homogeneous spaces of direct limit groups have already been used in [NRW01, Wol04], in the context of a Bott-Borel-Weil theorem, respectively direct limits of principal series representations. Universal complexifications of 'linear' direct limit groups $G \subseteq \mathrm{GL}_{\infty}(\mathbb{R})$ were discussed in [Glo02a], in the framework of Baker-Campbell-Hausdorff (BCH)-Lie groups. For special examples of direct limit manifolds of relevance for algebraic topology, see [KM97, $\S 47]$.

\section{Variants}

Although our main results concern the real and complex cases, some of the constructions apply just as well to Lie groups over local fields (i.e. totally disconnected, locally compact, non-discrete topological fields, such as the $p$-adic numbers), and are formulated accordingly. Readers mainly interested in the real and complex cases are invited to read ' $\mathbb{K}$ ' as $\mathbb{R}$ or $\mathbb{C}$, ignore the definition of smooth maps over general topological fields, and assume that all Lie groups are modelled on real or complex locally convex spaces.

\footnotetext{
${ }^{1}$ More generally, we can create direct limit Lie groups for arbitrary countable direct systems of finite-dimensional real or complex Lie groups. The bonding maps need not be injective.
} 


\section{FUndAMENTALS OF DIRECT LIMIT LIE THEORY}

\section{Basic definitions and facts}

We are working in two settings of differential calculus in parallel: (1) the Convenient Differential Calculus of Frölicher, Kriegl and Michor; and (2) Keller's $C_{c}^{\infty}$-theory (going back to Michal and Bastiani), as used, e.g., in [Mil82, Mil83, Glo02a, Glo02b], and generalized to a general differential calculus over topological fields in [BGN04]. For the basic notions of infinite-dimensional Lie theory $\left(L(G), \exp _{G}\right.$, logarithmic derivative, product integral), see [KM97] and [Mil83].

\subsection{Convenient Differential Calculus}

Our source for Convenient Differential Calculus is [KM97], and we presume familiarity with the basic ideas. The smooth maps and manifolds from convenient calculus will be called $c_{\mathbb{R}}^{\infty}$-maps and $c_{\mathbb{R}}^{\infty}$-manifolds here. Maps and manifolds which are holomorphic in the convenient sense will be called $c_{\mathbb{C}}^{\infty}$ or $c_{\mathbb{C}}^{\omega}$. Real analytic maps and manifolds in the convenient sense will be called $c_{\mathbb{R}}^{\omega}$. Likewise for Lie groups. The regular $c_{\mathbb{R}}^{\infty}$-Lie groups from convenient calculus (see [KM97, Definition 38.4]) will be called $c_{\mathbb{R}}^{\infty}$-regular; we call a $c_{\mathbb{C}}^{\infty}$-Lie group $c_{\mathbb{C}}^{\infty}$-regular or $c_{\mathbb{C}}^{\omega}$-regular if its underlying $c_{\mathbb{R}}^{\infty}$-Lie group is $c_{\mathbb{R}}^{\infty}$-regular. A $c_{\mathbb{R}}^{\omega}$-Lie group $G$ will be called $c_{\mathbb{R}}^{\omega}$-regular if it is $c_{\mathbb{R}}^{\infty}$-regular and the right product integral $\operatorname{Evol}_{G}^{r}(\gamma): \mathbb{R} \rightarrow G$ of each $c_{\mathbb{R}}^{\omega}$-curve $\gamma: \mathbb{R} \rightarrow L(G)$ is $c_{\mathbb{R}}^{\omega}$. The definitions of $c_{\mathbb{R}}^{\omega}$-regularity and $c_{\mathbb{C}}^{\omega}$-regularity ensure the following.

Lemma 1.2. Given $\mathbb{K} \in\{\mathbb{R}, \mathbb{C}\}$, let $G$ and $H$ be $c_{\mathbb{K}}^{\omega}$-Lie groups, where $G$ is simply connected and $H$ is $c_{\mathbb{K}}^{\omega}$-regular. Then, for every bounded $\mathbb{K}$-Lie algebra homomorphism $\alpha: L(G) \rightarrow L(H)$, there exists a unique $c_{\mathbb{K}}^{\omega}$-homomorphism $\beta: G \rightarrow H$ such that $L(\beta)=\alpha$.

Proof. By [KM97, Theorem 40.3], there exists a unique $c_{\mathbb{R}}^{\infty}$-homomorphism $\beta: G \rightarrow H$ such that $L(\beta)=\alpha$. If $\mathbb{K}=\mathbb{R}$ and $\gamma: \mathbb{R} \rightarrow G$ is a $c_{\mathbb{R}}^{\omega}$-curve, then $\beta \circ \gamma: \mathbb{R} \rightarrow H$ is a smooth curve with right logarithmic derivative $\delta^{r}(\beta \circ \gamma)=L(\beta) \circ \delta^{r} \gamma=\alpha \circ \delta^{r} \gamma$. Here $\alpha \circ \delta^{r} \gamma$ is $c_{\mathbb{R}}^{\omega}$, whence its right product integral $\beta \circ \gamma$ is $c_{\mathbb{R}}^{\omega}$, by $c_{\mathbb{R}}^{\omega}$-regularity. Hence, $\beta$ is $c_{\mathbb{R}}^{\omega}$. If $\mathbb{K}=\mathbb{C}$, then $\beta$ is a $c_{\mathbb{R}}^{\infty}$-homomorphism such that $T_{x}(\beta)$ is $\mathbb{C}$-linear for each $x \in G$, as $T_{1}(\beta)=\alpha$ is $\mathbb{C}$-linear. Hence, $\beta$ is $c_{\mathbb{C}}^{\omega}$ by $[\mathrm{KM} 97$, Theorem $7.19(8)]$.

\subsection{Keller's $C_{c}^{\infty}$-theory and analytic maps}

Let $E$ and $F$ be locally convex spaces over $\mathbb{K} \in\{\mathbb{R}, \mathbb{C}\}, U \subseteq E$ be open and $f: U \rightarrow F$ be a map. If $\mathbb{K}=\mathbb{R}$ and $r \in \mathbb{N}_{0} \cup\{\infty\}$, then $f$ is called $C_{\mathbb{R}}^{r}$ if it is continuous and, for all $k \in \mathbb{N}_{0}$ such that $k \leqslant r$, the iterated directional derivatives $d^{k} f\left(x, y_{1}, \ldots, y_{k}\right):=D_{y_{1}} \cdots D_{y_{k}} f(x)$ exist for all $x \in U$ and $y_{1}, \ldots, y_{k} \in E$, and define a continuous map $d^{k} f: U \times E^{k} \rightarrow F$. The $C_{\mathbb{R}}^{\infty}$-maps are also called smooth. If $\mathbb{K}=\mathbb{C}$, we call $f$ a $C_{\mathbb{C}}^{\infty}$-map, $C_{\mathbb{C}}^{\omega}$, or complex analytic, if it is continuous and given locally by a pointwise convergent series of continuous homogeneous polynomials [BS71, Definition 5.6]. If $\mathbb{K}=\mathbb{R}$, we call $f$ real analytic or $C_{\mathbb{R}}^{\omega}$ if it extends to a complex analytic map between open subsets of the complexifications of $E$ and $F$. See [Mil82, Mil83, Glo02b] for further information (also concerning the corresponding smooth and $\mathbb{K}$-analytic Lie groups and manifolds).

\subsection{General differential calculus}

Let $E$ and $F$ be (Hausdorff) topological vector spaces over a non-discrete topological field $\mathbb{K}, U \subseteq E$ be open, and $f: U \rightarrow F$ a map. According to [BGN04], $f$ is called $C_{\mathbb{K}}^{1}$ if it is continuous and there exists a (necessarily unique) continuous map $f^{[1]}: U^{[1]} \rightarrow F$ on $U^{[1]}:=\{(x, y, t) \in U \times E \times \mathbb{K}$ : $x+t y \in U\}$ such that $f^{[1]}(x, y, t)=(1 / t)(f(x+t y)-f(x))$ for all $(x, y, t) \in U^{[1]}$ such that $t \neq 0$. Inductively, $f$ is called $C_{\mathbb{K}}^{k+1}$ if it is $C_{\mathbb{K}}^{1}$ and $f^{[1]}$ is $C_{\mathbb{K}}^{k}$; it is $C_{\mathbb{K}}^{\infty}$ if it is $C_{\mathbb{K}}^{k}$ for all $k$. As shown in [BGN04], compositions of $C_{\mathbb{K}}^{k}$ maps are $C_{\mathbb{K}}^{k}$, and being $C_{\mathbb{K}}^{k}$ is a local property. For maps between open subsets of locally convex spaces, the present definitions of $C_{\mathbb{R}}^{k}$-maps and $C_{\mathbb{C}}^{\infty}$-maps are equivalent to those from $\S 1.3$ (see [BGN04, Propositions 7.4 and 7.7]). Analytic maps between open subsets 


\section{H. GLÖCKNER}

of Banach spaces over a complete valued field $\mathbb{K}$ (as used in [Bou89, Ser92]) are $C_{\mathbb{K}}^{\infty}$ (see [BGN04, Proposition 7.20]). For further information, also concerning $C_{\mathbb{K}}^{\infty}$-manifolds and Lie groups modelled on topological $\mathbb{K}$-vector spaces, see [BGN04, Glo03b, Glo03c, Glo04].

\subsection{Direct limits}

A direct system in a category $\mathbb{A}$ is a pair $\mathcal{S}=\left(\left(X_{i}\right)_{i \in I},\left(\phi_{i, j}\right)_{i \geqslant j}\right)$, where $(I, \leqslant)$ is a directed set, each $X_{i}$ an object of $\mathbb{A}$, and each $\phi_{i, j}: X_{j} \rightarrow X_{i}$ a morphism ('bonding map') such that $\phi_{i, i}=\operatorname{id}_{X_{i}}$ and $\phi_{i, j} \circ \phi_{j, k}=\phi_{i, k}$ if $i \geqslant j \geqslant k$. A cone over $\mathcal{S}$ is a pair $\left(X,\left(\phi_{i}\right)_{i \in I}\right)$, where $X \in$ ob $\mathbb{A}$ and $\phi_{i}: X_{i} \rightarrow X$ is a morphism for $i \in I$ such that $\phi_{i} \circ \phi_{i, j}=\phi_{j}$ if $i \geqslant j$. A cone $\left(X,\left(\phi_{i}\right)_{i \in I}\right)$ is a direct limit cone over $\mathcal{S}$ in the category $\mathbb{A}$ if, for every cone $\left(Y,\left(\psi_{i}\right)_{i \in I}\right)$ over $\mathcal{S}$, there exists a unique morphism $\psi: X \rightarrow Y$ such that $\psi \circ \phi_{i}=\psi_{i}$ for each $i$. We then write $\left(X,\left(\phi_{i}\right)_{i \in I}\right)=\lim _{\longrightarrow} \mathcal{S}$. If the bonding maps and 'limit maps' $\phi_{i}$ are understood, we simply call $X$ the direct limit of $\mathcal{S}$ and write $X=\lim _{\longrightarrow} X_{i}$. If $\mathcal{T}=\left(\left(Y_{i}\right)_{i \in I},\left(\psi_{i, j}\right)_{i \leqslant j}\right)$ is another direct system over $I$ and $\left(Y,\left(\psi_{i}\right)_{i \in I}\right)$ a cone over $\mathcal{T}$, we call a family $\left(\eta_{i}\right)_{i \in I}$ of morphisms $\eta_{i}: X_{i} \rightarrow Y_{i}$ compatible if $\eta_{i} \circ \phi_{i, j}=\psi_{i, j} \circ \eta_{j}$ for $i \geqslant j$. Then $\left(Y,\left(\psi_{i} \circ \eta_{i}\right)_{i \in I}\right)$ is a cone over $\mathcal{S}$; we write $\lim \eta_{i}:=\eta$ for the morphism $\eta: X \rightarrow Y$ such that $\eta \circ \phi_{i}=\psi_{i} \circ \eta_{i}$. If there is a compatible family $\left(\eta_{i}\right)_{i \in I}$ with each $\eta_{i}$ an isomorphism, $\mathcal{S}$ and $\mathcal{T}$ are called equivalent. Then $\mathcal{S}$ has a direct limit if and only if $\mathcal{T}$ does; in this case, $\lim \eta_{i}$ is an isomorphism. Every countable direct set has a cofinal subsequence, whence countable direct systems can be replaced by direct sequences, namely $I=(\mathbb{N}, \leqslant)$.

\subsection{Direct limits of sets, topological spaces, and groups}

If $\mathcal{S}=\left(\left(X_{i}\right)_{i \in I},\left(\phi_{i, j}\right)_{i \geqslant j}\right)$ is a direct system of sets, write $(j, x) \sim(k, y)$ if there exists $i \geqslant j, k$ such that $\phi_{i, j}(x)=\phi_{i, k}(y)$; then $X:=\left(\coprod_{i \in I} X_{i}\right) / \sim$, together with the maps $\phi_{i}: X_{i} \rightarrow X, \phi_{i}(x):=[(i, x)]$, is the direct limit of $\mathcal{S}$ in the category of sets. Here $X=\bigcup_{i \in I} \phi_{i}\left(X_{i}\right)$. If each $\phi_{i, j}$ is injective, then so is each $\phi_{i}$, whence $\mathcal{S}$ is equivalent to the direct system of the subsets $\phi_{i}\left(X_{i}\right) \subseteq X$, together with the inclusion maps. This facilitates to replace injective direct systems by direct systems in which all bonding maps are inclusion maps. If $\mathcal{S}:=\left(\left(X_{i}\right)_{i \in I},\left(\phi_{i, j}\right)\right)$ is a direct system of topological spaces and continuous maps, then the direct limit $\left(X,\left(\phi_{i}\right)_{i \in I}\right)$ of the underlying sets becomes the direct limit in the category of topological spaces and continuous maps if we equip $X$ with the $D L$-topology, the final topology with respect to the family $\left(\phi_{i}\right)_{i \in I}$. Thus, $U \subseteq X$ is open if and only if $\phi_{i}^{-1}(U)$ is open in $X_{i}$, for each $i$. If $\mathcal{S}$ is strict in the sense that each $\phi_{i, j}$ is a topological embedding, then each $\phi_{i}$ is also a topological embedding [NRW93, Lemma A.5]. If $\left(\left(G_{i}\right)_{i \in I},\left(\phi_{i, j}\right)_{i \geqslant j}\right)$ is a direct system of groups and homomorphisms, then the direct limit $\left(G,\left(\phi_{i}\right)_{i \in I}\right)$ of the underlying sets becomes the direct limit in the category of groups and homomorphisms when equipped with the unique group structure making each $\phi_{i}$ a homomorphism; the group inversion and multiplication on $G$ are $\lim _{\longrightarrow} \kappa_{i}$ and $\lim _{\longrightarrow} \mu_{i}$, in terms of those on the $G_{i}$.

For further information concerning direct limits of topological groups and topological spaces, see [Glo03a, Han71, HST01, TSH98].

Lemma 1.7. Let $X_{1} \subseteq X_{2} \subseteq \cdots$ be an ascending sequence of topological spaces such that the inclusion maps are continuous; equip $X:=\bigcup_{n \in \mathbb{N}} X_{n}$ with the final topology with respect to the inclusion maps $\lambda_{n}: X_{n} \rightarrow X$ (the DL-topology). Then the following hold.

(a) If each $X_{n}$ is $T_{1}$, then so is $X$.

(b) If $U_{n} \subseteq X_{n}$ is open and $U_{1} \subseteq U_{2} \subseteq \cdots$, then $U:=\bigcup_{n} U_{n}$ is open in $X$ and the DL-topology on $U=\underline{\lim } U_{n}$ coincides with the topology induced by $X$.

(c) If each $X_{n}$ is locally compact, then $X$ is Hausdorff.

(d) If each $X_{n}$ is $T_{1}$ and $K \subseteq X$ is compact, then $K \subseteq X_{n}$ for some $n$. 


\section{FUndAMENTALS OF DIRECT LIMIT LIE THEORY}

Proof. (a) Let $x \in X$. Then $\lambda_{n}^{-1}(\{x\})$ is either $\{x\}$ or empty, hence closed in the $T_{1}$-space $X_{n}$. Hence, $\{x\}$ is closed in $X$.

(b) and (c) This is proved in [Han71, Proposition 4.1(ii)] and [Glo03a, Lemma 3.1] for strict direct sequences, but the strictness is not used in the proofs.

(d) If not, for each $n$ we find $x_{n} \in K \backslash X_{n}$. Then $D:=\left\{x_{n}: n \in \mathbb{N}\right\} \subseteq K$ is closed in $X$ (and thus compact), as $D \cap X_{n}$ is finite and thus closed, for each $n$. On the other hand, $D=\underline{\lim }\left(D \cap X_{n}\right)$ for the topology induced by $X$, as $D$ is closed in $X$. Now $D \cap X_{n}$ being discrete, this entails $D$ is discrete and hence finite (being also compact) - contradiction.

1.8 Let $E$ be a countable-dimensional vector space over a non-discrete, locally compact topological field $\mathbb{K}$ (e.g. $\mathbb{K}=\mathbb{R}$ or $\mathbb{C}$ ). Then the finest vector topology on $E$ is locally convex and coincides with the so-called finite topology, the final topology with respect to the inclusion maps $F \rightarrow E$, where $F$ ranges through the set of finite-dimensional vector subspaces of $E$ (and $F$ is equipped with its canonical Hausdorff vector topology). Thus, the finite topology on $E$ is the DL-topology on $E=\lim F$. See [Glo03a] and the references therein for these standard facts. The space $\mathbb{K}^{\infty}:=\mathbb{K}^{(\mathbb{N})}=\underset{\lim }{\longrightarrow} \mathbb{K}^{n}$ of finite sequences will always be equipped with the finite topology. We shall frequently identify $\mathbb{K}^{n}$ with the subspace $\mathbb{K}^{n} \times\{0\}$ of $\mathbb{K}^{\infty}$, and $\mathbb{K}^{m}$ with $\mathbb{K}^{m} \times\{0\} \subseteq \mathbb{K}^{n}$ if $n \geqslant m$.

Lemma 1.9. Let $\mathbb{K}$ be $\mathbb{R}, \mathbb{C}$ or a local field, and $E$ be a $\mathbb{K}$-vector space of countable dimension, equipped with the finite topology. Let $E_{1} \subseteq E_{2} \subseteq \cdots$ be an ascending sequence of finite-dimensional vector subspaces of $E$ such that $\bigcup_{n \in \mathbb{N}} E_{n}=E$, and $U_{n} \subseteq E_{n}$ be open sets such that $U_{1} \subseteq U_{2} \subseteq \cdots$. Let $f: U \rightarrow F$ be a map to a topological $\mathbb{K}$-vector space $F$, defined on the open subset $U:=\bigcup_{n \in \mathbb{N}} U_{n}$ of $E$. Then the following hold.

(a) Given $r \in \mathbb{N}_{0} \cup\{\infty\}, f$ is $C_{\mathbb{K}}^{r}$ if and only if $f_{n}:=\left.f\right|_{U_{n}}: U_{n} \rightarrow F$ is $C_{\mathbb{K}}^{r}$ for each $n$.

(b) If $\mathbb{K} \in\{\mathbb{R}, \mathbb{C}\}$ and $F$ is locally convex and Mackey complete, then $f$ is $C_{\mathbb{K}}^{\infty}$ if and only if it is $c_{\mathbb{K}}^{\infty}$. Furthermore, $f$ is $c_{\mathbb{K}}^{\omega}$ if and only if $\left.f\right|_{U_{n}}$ is $c_{\mathbb{K}}^{\omega}$ for each $n \in \mathbb{N}$.

Proof. (a) For $\mathbb{K}=\mathbb{R}$ and locally convex $F$, the assertion is covered by [Glo03a, lines preceding Lemma 4.1]. This also implies the claim for $\mathbb{K}=\mathbb{C}, r=\infty, F$ locally convex because then $f$ is $C_{\mathbb{R}}^{\infty}$ with $d f(x, \bullet)$ complex linear for each $x$ (since $\left.\left.d f(x, \bullet)\right|_{E_{n}}=d f_{n}(x, \bullet)\right)$, whence $f$ is complex analytic by [Glo02b, Lemma 2.5].

To prove the general case, we may assume that $r<\infty$. Lemma 1.7(b) settles the case $r=0$. If $r \geqslant 1$, note that $U_{1}^{[1]} \subseteq U_{2}^{[1]} \subseteq \cdots$ and $U^{[1]}=\bigcup_{n} U_{n}^{[1]}$. The product topology on $E \times E \times \mathbb{K}$ is the finite topology (cf. [Glo03a, Proposition 3.3]) and hence induces on $U^{[1]}$ the topology making it the direct limit topological space $U^{[1]}=\lim _{n} U_{n}^{[1]}$ (Lemma 1.7(b)). By induction, the cone $\left(F,\left(f_{n}^{[1]}\right)_{n \in \mathbb{N}}\right)$ of $C_{\mathbb{K}}^{r-1}$-maps induces a $C_{\mathbb{K}}^{r-1}$-map $g: U^{[1]}=\lim _{n} U_{n}^{[1]} \rightarrow F$, determined by $\left.g\right|_{U_{n}^{[1]}}=f_{n}^{[1]}$. As $g$ is continuous and extends the difference quotient map, $f$ is $C_{\mathbb{K}}^{1}$ with $f^{[1]}=g$. Now $f$ being $C_{\mathbb{K}}^{1}$ with $f^{[1]}=g$ of class $C_{\mathbb{K}}^{r-1}$, the map $f$ is $C_{\mathbb{K}}^{r}$.

(b) If $f$ is $C_{\mathbb{K}}^{\infty}$, then it is $c_{\mathbb{K}}^{\infty}$. If $f$ is $c_{\mathbb{K}}^{\infty}$, then $\left.f\right|_{U_{n}}$ is $c_{\mathbb{K}}^{\infty}$ for each $n$ and thus $C_{\mathbb{K}}^{\infty}$, as $\operatorname{dim}_{\mathbb{K}}\left(E_{n}\right)<\infty$. Hence $f$ is $C_{\mathbb{K}}^{\infty}$, by (a). Given a $c_{\mathbb{R}}^{\omega}$-curve $\gamma: \mathbb{R} \rightarrow U$ and $t_{0} \in \mathbb{R}$, pick an open relatively compact neighbourhood $J \subseteq \mathbb{R}$ of $t_{0}$. Then $\gamma(J) \subseteq U_{n}$ for some $n$ by Lemma $1.7(\mathrm{~d})$, and thus $\left.\gamma\right|_{J}$ is $c_{\mathbb{R}}^{\omega}$ if $\left.f\right|_{U_{n}}$ is. The remainder is now obvious.

A map $f: \mathbb{R}^{\infty} \rightarrow \mathbb{R}$ which is $C_{\mathbb{R}}^{\omega}$ on each $\mathbb{R}^{n}$ need not be $C_{\mathbb{R}}^{\omega}$ [KM97, Example 10.8]. For this reason, we have to work with the weaker concept of $c_{\mathbb{R}}^{\omega}$-maps. 


\section{H. GLÖCKNER}

\section{Extension of charts}

In this section, we explain how a chart of a submanifold $M_{1} \subseteq M_{2}$ (or its restriction to a slightly smaller open set) can be extended to a chart of $M_{2}$.

Lemma 2.1. Let $M_{1}$ and $M_{2}$ be finite-dimensional smooth (respectively real analytic) manifolds over $\mathbb{R}$, of dimensions $m_{1}$ and $m_{2}$, respectively. Assume that $M_{1} \subseteq M_{2}$ and assume that the inclusion map $\lambda: M_{1} \rightarrow M_{2}$ is a smooth (respectively real analytic) immersion. Let $\phi_{1}: U_{1} \rightarrow V_{1}$ be a chart of $M_{1}$, where $U_{1}$ is open in $\mathbb{R}^{m_{1}}$ and $V_{1}$ is an open, relatively compact, contractible subset of $M_{1}$. Then there exists a chart $\phi_{2}: U_{2} \rightarrow V_{2}$ of $M_{2}$ such that $U_{2} \cap\left(\mathbb{R}^{m_{1}} \times\{0\}\right)=U_{1} \times\{0\}, \phi_{2}(x, 0)=\phi_{1}(x)$ for all $x \in U_{1}$, and such that $V_{2} \subseteq M_{2}$ is relatively compact and contractible.

Proof. Because $C:=\overline{V_{1}} \subseteq M_{1}$ is compact, the map $\left.\lambda\right|_{C}$ is a topological embedding. Now $V_{1}$ being open in $C$, we deduce that $V_{1}=\left.\lambda\right|_{C}\left(V_{1}\right)$ is open in $\lambda(C)$, whence there exists an open subset $W \subseteq M_{2}$ such that $W \cap \lambda(C)=V_{1}$. Since $\lambda(C)$ is closed in $M_{2}$, the preceding formula shows that $V_{1}$ is closed in $W$. After shrinking $W$, we may assume that $W$ is $\sigma$-compact, and relatively compact in $M_{2}$. Then $V_{1}$ is a closed submanifold of the $\sigma$-compact, relatively compact, open submanifold $W$ of $M_{2}$.

Smooth case. By [Lan99, Theorem IV.5.1], $V_{1}$ admits a smooth tubular neighbourhood in $W$, i.e. there exists a $C_{\mathbb{R}}^{\infty}$-diffeomorphism $\psi: V_{2} \rightarrow P$ from some open neighbourhood $V_{2}$ of $V_{1}$ in $W$ onto some open neighbourhood $P$ of the zero-section of some smooth vector bundle $\pi: E \rightarrow V_{1}$ over $V_{1}$, such that $\left.\psi\right|_{V_{1}}=\mathrm{id}_{V_{1}}$ (identifying $V_{1}$ with the zero-section of $E$ ).

Real analytic case. Being $\sigma$-compact, $W$ is $C_{\mathbb{R}}^{\omega}$-diffeomorphic to a closed real analytic submanifold of $\mathbb{R}^{k}$ for some $k \in \mathbb{N}_{0}$ (see [Gra58b, Theorem 3]), whence $W$ admits a real analytic Riemannian metric $g$. Using the real analytic Riemannian metric, the classical construction of tubular neighbourhoods provides a real analytic tubular neighbourhood $\psi: V_{1} \supseteq V_{2} \rightarrow P \subseteq E$.

In either case, after shrinking $V_{2}$ and $P$, we may assume that $P$ is balanced, i.e. $[-1,1] P \subseteq P$ (using the scalar multiplication in the fibres of $E$ ). Being a vector bundle over a contractible, $\sigma$-compact base manifold, $E$ is trivial. This is well known in the smooth case [Hir76, Corollary 4.2.5]. For the real analytic case, note that $E$ is associated to a real analytic $\mathrm{GL}(F)$-principal bundle over the $\sigma$-compact, contractible $C_{\mathbb{R}}^{\omega}$-manifold $V_{1}$, where $F:=\mathbb{R}^{m_{2}-m_{1}}$ is the fibre of $E$. This principal bundle is trivial by [Tog67, Teorema 5] (combined with [Hir76, Corollary 4.2.5]), and hence so is $E$. (Compare also [Anc76, Gua02].)

By the preceding, we find an isomorphism of smooth (respectively real analytic) vector bundles $\theta: E \rightarrow V_{1} \times \mathbb{R}^{m_{2}-m_{1}}$. Then $\kappa: \phi_{1}^{-1} \times$ id $: V_{1} \times \mathbb{R}^{m_{2}-m_{1}} \rightarrow U_{1} \times \mathbb{R}^{m_{2}-m_{1}} \subseteq \mathbb{R}^{m_{2}}$ is a $C_{\mathbb{R}}^{\infty}$ - (respectively $\left.C_{\mathbb{R}^{-}}^{\omega}\right)$ diffeomorphism, and $U_{2}:=\kappa(\theta(P))$ is an open subset of $\mathbb{R}^{m_{2}}$ such that $U_{2} \cap\left(\mathbb{R}^{m_{1}} \times\{0\}\right)=U_{1}$. Then $\phi_{2}:=\left.(\kappa \circ \theta \circ \psi)^{-1}\right|_{U_{2}} ^{V_{2}}: U_{2} \rightarrow V_{2}$ is a $C_{\mathbb{R}^{\infty}}^{\infty}$ (respectively $C_{\mathbb{R}^{-}}^{\omega}$ ) diffeomorphism from $U_{2}$ onto the open subset $V_{2}$ of $M_{2}$, such that $\phi_{2}(x, 0)=\phi_{1}(x)$ for all $x \in U_{1}$. Since $V_{2} \subseteq W$, the set $V_{2}$ is relatively compact in $M_{2}$. To see that $V_{2}$ is contractible, we only need to show that so is $P$, as $V_{2}$ and $P$ are homeomorphic. Let $H:[0,1] \times V_{1} \rightarrow V_{1}$ be a homotopy from id $V_{V_{1}}$ to a constant map. The map $[0,1] \times P \rightarrow P,(t, x) \mapsto(1-t) x$ (which uses scalar multiplication in the fibres) is a homotopy from $\operatorname{id}_{P}$ to $\left.\pi\right|_{P}$. The map $[0,1] \times P \rightarrow P,(t, x) \mapsto H(t, \pi(x))$ is a homotopy from $\left.\pi\right|_{P}$ to a constant map. Thus, $\operatorname{id}_{P}$ is homotopic to a constant map and thus $P$ is contractible.

Definition 2.2. Let $\mathbb{K}$ be $\mathbb{R}, \mathbb{C}$ or a local field, and $|\cdot|$ be an absolute value on $\mathbb{K}$ defining its topology. Given $n \in \mathbb{N}_{0}$ and $r>0$, we let

$$
\Delta_{r}^{n}:=\left\{\left(x_{1}, \ldots, x_{n}\right) \in \mathbb{K}^{n}:\left|x_{j}\right|<r \text { for all } j=1, \ldots, n\right\}
$$

be the $n$-dimensional polydisk of radius $r$ around 0 . If we wish to emphasize the ground field, we also write $\Delta_{r}^{n}(\mathbb{K})$ for $\Delta_{r}^{n}$. 


\section{FUndAMENTALS OF DIRECT LIMIT LIE THEORY}

If $\mathbb{K}$ is a local field, we define $C_{\mathbb{K}}^{\infty}$-immersions (and $C_{\mathbb{K}}^{\infty}$-submersions) between finite-dimensional $C_{\mathbb{K}}^{\infty}$-manifolds analogous to the $\mathbb{K}$-analytic case [Ser92]. Because an inverse function theorem holds for $C_{\mathbb{K}}^{\infty}$-maps [Glo03b], $C_{\mathbb{K}}^{\infty}$-immersions and submersions have the usual properties.

Lemma 2.3 (Extension Lemma). Let $\mathbb{K}$ be $\mathbb{R}, \mathbb{C}$ or a local field. Let $M$ be a finite-dimensional $C_{\mathbb{K}}^{\infty}$-manifold (or a finite-dimensional real analytic manifold), of dimension $m \in \mathbb{N}_{0}$, and $\phi: \Delta_{r}^{n} \rightarrow M$ be a $C_{\mathbb{K}}^{\infty}$ (respectively real analytic) injective immersion, where $n \in\{0,1, \ldots, m\}$ and $r>0$. Then, for every $s \in] 0, r\left[\right.$, there exists a $C_{\mathbb{K}}^{\infty}$-diffeomorphism (respectively a real analytic diffeomorphism) $\psi: \Delta_{s}^{m} \rightarrow V$ onto an open subset $V$ of $M$ such that $\psi(x, 0)=\phi(x)$ for all $x \in \Delta_{s}^{n}$. If $\mathbb{K}$ is a local field, the conclusion remains valid for $s=r$. The subset $V \subseteq M$ can be chosen relatively compact.

Proof. Let $s \in] 0, r[$ and $t \in] s, r[$.

The case of smooth or analytic manifolds over $\mathbb{K}=\mathbb{R}$. We equip $M_{1}:=\phi\left(\Delta_{r}^{n}\right)$ with the smooth (respectively real analytic) manifold structure making $\left.\phi\right|^{M_{1}}: \Delta_{r}^{n} \rightarrow M_{1}$ a diffeomorphism. Then the inclusion map $\lambda: M_{1} \rightarrow M$ is an immersion, $V_{1}:=\phi\left(\Delta_{t}^{n}\right)$ is a relatively compact, contractible, $\sigma$-compact open subset of $M_{1}$, and $\phi_{1}:=\left.\phi\right|_{\Delta_{t}^{n}} ^{V_{1}}: \Delta_{t}^{n} \rightarrow V_{1}$ is a chart for $M_{1}$. By Lemma 2.1, there exists a $C_{\mathbb{R}}^{\infty}$ - (respectively $C_{\mathbb{R}^{-}}^{\omega}$ ) diffeomorphism $\phi_{2}: U_{2} \rightarrow V_{2}$ from an open subset $U_{2}$ of $\mathbb{R}^{m}$ onto an open subset $V_{2}$ of $M$ such that $U_{2} \cap\left(\mathbb{R}^{n} \times\{0\}\right)=\Delta_{t}^{n} \times\{0\}$ and $\phi_{2}(x, 0)=\phi_{1}(x)=\phi(x)$ for all $x \in \Delta_{t}^{n}$. Now $\overline{\Delta_{s}^{n}} \subseteq \mathbb{R}^{n}$ being compact, we find $\varepsilon>0$ such that $\overline{\Delta_{s}^{n}} \times \Delta_{\varepsilon}^{m-n} \subseteq U_{2}$. Then

$$
\psi: \Delta_{s}^{m} \rightarrow M, \quad \psi(x, y):=\phi_{2}\left(x, \frac{\varepsilon}{s} y\right) \quad \text { for } x \in \Delta_{s}^{n}, y \in \Delta_{s}^{m-n}
$$

is a mapping with the required properties.

The case $\mathbb{K}=\mathbb{C}$. The map $\left.\phi\right|_{\Delta_{t}^{n}}$ is an embedding of complex manifolds, whence so is $f: \Delta_{1}^{n} \rightarrow M, f(x):=\phi(t x)$. By [Roy74, Proposition 1], there exists a holomorphic embedding $F: \Delta_{s / t}^{m} \times \Delta_{1}^{n-m} \rightarrow M$ such that $F(x, 0)=f(x)$ for all $x \in \Delta_{s / t}^{n}$. Then $\psi: \Delta_{s}^{m} \rightarrow M, \psi(x, y):=$ $F\left((1 / t) x,(1 / s) y\right.$ ) (where $x \in \Delta_{s}^{n}, y \in \Delta_{s}^{m-n}$ ) is a holomorphic embedding with the desired properties.

Relative compactness of $V$. By the real or complex case already discussed, there exists an extension $\widetilde{\psi}: \Delta_{t}^{m} \rightarrow \widetilde{V}$ of $\left.\phi\right|_{\Delta_{t}^{n}}$. Then $V:=\widetilde{\psi}\left(\Delta_{s}^{m}\right)$ is a relatively compact open subset of $M$, and $\psi:=\left.\widetilde{\psi}\right|_{\Delta_{s}^{m}} ^{V}$ has the desired properties.

The case where $\mathbb{K}$ is a local field. In this case, $\Delta_{r}^{n}$ is compact, whence $\phi$ is a $C_{\mathbb{K}}^{\infty}$-diffeomorphism from $\Delta_{r}^{n}$ onto the compact $C_{\mathbb{K}}^{\infty}$-submanifold $M_{1}:=\operatorname{im} \phi$ of $M$. The proof of [Glo03a, Lemma 8.1] (tackling the $\mathbb{K}$-analytic case) carries over verbatim to the case of $C_{\mathbb{K}}^{\infty}$-manifolds; we therefore find a $C_{\mathbb{K}}^{\infty}$-diffeomorphism $\theta: \Delta_{r}^{n} \times \mathbb{O}^{m-n} \rightarrow M$ such that $\theta(x, 0)=\phi(x)$, where $\mathbb{O}$ is the maximal compact subring of $\mathbb{K}$. Pick $a \in \mathbb{K}^{\times}$such that $a \Delta_{r}^{m-n} \subseteq \mathbb{O}^{m-n}$; then $\psi: \Delta_{r}^{m} \rightarrow M, \psi(x, y):=\theta(x, a y)$ for $x \in \Delta_{r}^{n}, y \in \Delta_{r}^{m-n}$ (respectively its restriction to $\Delta_{s}^{m}$ ) is the required chart for $M$.

\section{Direct limits of finite-dimensional manifolds}

Let $\mathbb{K}$ be $\mathbb{R}, \mathbb{C}$ or a local field. Throughout this section, we let $\mathcal{S}:=\left(\left(M_{i}\right)_{i \in I},\left(\lambda_{i, j}\right)_{i \geqslant j}\right)$ be a direct system of finite-dimensional $C_{\mathbb{K}}^{\infty}$-manifolds $M_{i}$ and injective $C_{\mathbb{K}}^{\infty}$-immersions $\lambda_{i, j}: M_{j} \rightarrow M_{i}$. We let $\left(M,\left(\lambda_{i}\right)_{i \in I}\right)$ be the direct limit of $\mathcal{S}$ in the category of topological spaces, and abbreviate $s:=\sup \left\{\operatorname{dim}_{\mathbb{K}}\left(M_{i}\right): i \in I\right\} \in \mathbb{N}_{0} \cup\{\infty\}$. Our goal is to make $M$ a manifold, and study its basic properties.

Theorem 3.1. There exists a uniquely determined $C_{\mathbb{K}}^{\infty}$-manifold structure on $M$, modelled on the complete, locally convex topological $\mathbb{K}$-vector space $\mathbb{K}^{s}$, which makes $\lambda_{i}: M_{i} \rightarrow M$ a $C_{\mathbb{K}}^{\infty}$-map, 


\section{H. GLÖCKNeR}

for each $i \in I$, and such that $\left(M,\left(\lambda_{i}\right)_{i \in I}\right)=\lim \mathcal{S}$ in the category of $C_{\mathbb{K}}^{\infty}$-manifolds modelled on topological $\mathbb{K}$-vector spaces (and $C_{\mathbb{K}}^{\infty}$-maps). For each $i \in I$ and $x \in M_{i}$, the differential $T_{x}\left(\lambda_{i}\right): T_{x}\left(M_{i}\right) \rightarrow T_{\lambda_{i}(x)}(M)$ is injective. For each $r \in \mathbb{N}_{0}$, the $C_{\mathbb{K}}^{r}$-manifold underlying $M$ satisfies $\left(M,\left(\lambda_{i}\right)_{i \in I}\right)=\lim _{\longrightarrow} \mathcal{S}$ in the category of $C_{\mathbb{K}}^{r}$-manifolds modelled on topological $\mathbb{K}$-vector spaces.

Proof. After passing to a cofinal subsequence of an equivalent direct system (cf. $\S 1.6$ ), we may assume without loss of generality that $I=\mathbb{N}, M_{1} \subseteq M_{2} \subseteq \cdots$, and that the immersion $\lambda_{n, m}$ is the inclusion map for all $n, m \in \mathbb{N}$ such that $n \geqslant m$. We let $M:=\bigcup_{n \in \mathbb{N}} M_{n}$, equipped with the final topology with respect to the inclusion maps $\lambda_{n}: M_{n} \rightarrow M$; then $\left(M,\left(\lambda_{n}\right)_{n \in \mathbb{N}}\right)=\underline{\lim }\left(\left(M_{n}\right),\left(\lambda_{n, m}\right)\right)$ in the category of topological spaces. We abbreviate $d_{n}:=\operatorname{dim}_{\mathbb{K}}\left(M_{n}\right)$ and $c_{n}:=\overrightarrow{d_{n+1}}-d_{n}$.

Let $\mathcal{A}$ be the set of all maps $\phi: P_{\phi} \rightarrow Q_{\phi} \subseteq M$ such that $P_{\phi}=\bigcup_{n \in \mathbb{N}} P_{n} \subseteq \mathbb{K}^{s}, Q_{\phi}=\bigcup_{n \in \mathbb{N}} Q_{n}$, and $\phi=\lim _{\longrightarrow} \phi_{n}$ for some sequence $\left(\phi_{n}\right)_{n \in \mathbb{N}}$ of charts $\phi_{n}: P_{n} \rightarrow Q_{n} \subseteq M_{n}$, where each $P_{n}$ is an open (possibly empty) subset of $\mathbb{K}^{d_{n}}, Q_{n}$ open in $M_{n}$, and $Q_{m} \subseteq Q_{n}$ and $\left.\phi_{n}\right|_{Q_{m}}=\phi_{m}$ whenever $n \geqslant m$. Here Lemma 1.7(b) allows us to interpret the open subsets $P_{\phi} \subseteq \mathbb{K}^{s}$ and $Q_{\phi} \subseteq M$ as the direct $\operatorname{limits} \underset{\lim }{\longrightarrow} Q_{n}$ and $\lim _{\longrightarrow} P_{n}$ in the category of topological spaces, whence $\phi$ is continuous. Because each $\phi_{n}$ is injective, $\phi$ is also injective, and furthermore $\phi$ is surjective, by definition of $Q_{\phi}$. If $V \subseteq P_{\phi}$ is open, then $V \cap P_{n}$ is open in $P_{n}$, whence $S_{n}:=\phi_{n}\left(V \cap P_{n}\right)$ is open in $Q_{n}$. Because $S_{1} \subseteq S_{2} \subseteq \cdots$, the union $\phi(V)=\bigcup_{n \in \mathbb{N}} S_{n}$ is open in $Q_{\phi}$ (Lemma 1.7(b)). Thus, $\phi$ is an open map. We have shown that $\phi$ is a homeomorphism.

We claim that $\mathcal{A}$ is a $C_{\mathbb{K}}^{\infty}$-atlas for $M$. We first show that $\bigcup_{\phi \in \mathcal{A}} Q_{\phi}=M$. To this end, let $x \in M$. Then there exists $\ell \in \mathbb{N}_{0}$ such that $x \in M_{\ell}$. Define $r_{n}:=1+2^{-n}$ for $n \in \mathbb{N}$. We let $\phi_{n}: P_{n} \rightarrow Q_{n}$ be the chart of $M_{n}$ with $P_{n}:=Q_{n}:=\emptyset$, for all $n<\ell$. We pick a chart $\psi_{\ell}: \Delta_{r_{\ell}}^{d_{\ell}}(\mathbb{K}) \rightarrow W_{\ell} \subseteq M_{\ell}$ of $M_{\ell}$ around $x$, such that $\psi_{\ell}(0)=x$. Inductively, the Extension Lemma 2.3 provides charts $\psi_{n}$ : $\Delta_{r_{n}}^{d_{n}} \rightarrow W_{n} \subseteq M_{n}$ for $n \in\{\ell+1, \ell+2, \ldots\}$ such that $\left.\psi_{n}\right|_{\Delta_{r_{n}}^{d_{n-1}}}=\left.\psi_{n-1}\right|_{\Delta_{r_{n}}^{d_{n-1}}}$ (identifying $\mathbb{K}^{d_{n-1}}$ with $\left.\mathbb{K}^{d_{n-1}} \times\{0\} \subseteq \mathbb{K}^{d_{n}}\right)$. Define $P_{n}:=\Delta_{1}^{d_{n}}, Q_{n}:=\psi_{n}\left(P_{n}\right)$, and $\phi_{n}:=\left.\psi_{n}\right|_{P_{n}} ^{Q_{n}}: P_{n} \rightarrow Q_{n}$ for $n \geqslant \ell$. Then $P_{\phi}:=\bigcup_{n \in \mathbb{N}} P_{n}$ is open in $\mathbb{K}^{s}, Q_{\phi}:=\bigcup_{n \in \mathbb{N}} Q_{n}$ is open in $M$, and $\phi:=\lim _{n} \phi_{n}: P_{\phi} \rightarrow Q_{\phi}$ is an element of $\mathcal{A}$, with $x \in Q_{\phi}$, as desired.

Compatibility of the charts. Assume that $\phi:=\lim \phi_{n}: P_{\phi} \rightarrow Q_{\phi}$ and $\psi:=\lim \psi_{n}: P_{\psi} \rightarrow Q_{\psi}$ are elements of $\mathcal{A}$, where $\phi_{n}: P_{n} \rightarrow Q_{n}$ and $\psi_{n}: A_{n} \longrightarrow B_{n}$. Let $x \in \phi^{-1}\left(Q_{\psi}\right)$. Then $\phi(x) \in Q_{\phi} \cap Q_{\psi}$, entailing that there exists $\ell \in \mathbb{N}$ such that $\phi(x) \in Q_{\ell} \cap B_{\ell}$. Then $x \in P_{n} \cap \phi_{n}^{-1}\left(B_{n}\right)=: X_{n}$ for all $n \geqslant \ell$. Since $X_{n}$ is open in $\mathbb{K}^{d_{n}}$ and $X_{\ell} \subseteq X_{\ell+1} \subseteq \cdots$, the union $X:=\bigcup_{n \geqslant \ell} X_{n}$ is open in $\mathbb{K}^{s}$. Furthermore, the coordinate changes $\tau_{n}:=\left.\left.\psi_{n}^{-1}\right|_{Q_{n} \cap B_{n}} \circ \phi_{n}\right|_{X_{n}}: X_{n} \rightarrow \psi_{n}^{-1}\left(Q_{n}\right)=: Y_{n}$ are $C_{\mathbb{K}}^{\infty}$-diffeomorphisms, for all $n \geqslant \ell$. By Lemma 1.9(a), the map $\left.\left.\psi^{-1}\right|_{\phi(X)} ^{Y} \circ \phi\right|_{X}=\lim _{\longrightarrow} \geqslant \ell \tau_{n}: X \rightarrow \bigcup_{n \geqslant \ell} Y_{n}=: Y$ is $C_{\mathbb{K}}^{\infty}$, entailing that the bijection $\tau:=\left.\left.\psi^{-1}\right|_{Q_{\phi} \cap Q_{\psi}} \circ \phi\right|_{\phi^{-1}\left(Q_{\psi}\right)}: \phi^{-1}\left(Q_{\psi}\right) \rightarrow \psi^{-1}\left(Q_{\phi}\right)$ is $C_{\mathbb{K}}^{\infty}$ on some open neighbourhood of $x$. As $x$ was arbitrary, $\tau$ is $C_{\mathbb{K}}^{\infty}$ and the same reasoning shows that so is $\tau^{-1}$. Thus $\mathcal{A}$ is an atlas making $M$ a $C_{\mathbb{K}}^{\infty}$-manifold modelled on $\mathbb{K}^{s}$.

Each $\lambda_{n}$ is smooth. To see this, assume that $n \in \mathbb{N}$ and $x \in M_{n}$. As just shown, there exists a chart $\phi: P_{\phi} \rightarrow Q_{\phi}$ in $\mathcal{A}$, say $\phi=\lim _{\phi_{k}}$ with charts $\phi_{k}: P_{k} \rightarrow Q_{k} \subseteq M_{k}$ for $k \in \mathbb{N}$, such that $x \in P_{n}$. Then $\phi^{-1} \circ \lambda_{n} \circ \phi_{n}=\phi^{-1} \circ \phi_{n}: \mathbb{K}^{d_{n}} \supseteq P_{n} \rightarrow P \subseteq \mathbb{K}^{s}$ is the inclusion map and hence smooth, and its differential at $x$ is injective. Hence $\lambda_{n}$ is smooth on the open neighbourhood $Q_{n}$ of $x$, and $T_{x}\left(\lambda_{n}\right)$ is injective. As $x$ was arbitrary, $\lambda_{n}$ is smooth.

Direct limit property and uniqueness. Fix $r \in \mathbb{N}_{0} \cup\{\infty\}$. Assume that $Y$ is a $C_{\mathbb{K}}^{r}$-manifold modelled on a topological $\mathbb{K}$-vector space $E$ and $f_{n}: M_{n} \rightarrow Y$ a $C_{\mathbb{K}}^{r}$-map for each $n \in \mathbb{N}$ such that $\left(Y,\left(f_{n}\right)_{n \in \mathbb{N}}\right)$ is a cone over $\mathcal{S}$; thus $\left.f_{n}\right|_{M_{m}}=f_{m}$ if $n \geqslant m$. Then there is a uniquely determined map $f: M \rightarrow Y$ such that $\left.f\right|_{M_{n}}=f_{n}$ for all $n \in \mathbb{N}$. Since $M=\lim M_{n}$ as a topological space, $f$ is continuous. If $x \in M$, we find a chart $\phi: P_{\phi} \rightarrow Q_{\phi}$ of $M$ around $\vec{x}$ in the atlas $\mathcal{A}$, where $\phi=\underline{\lim } \phi_{n}$ 


\section{FundAMENTALS OF DIRECT LIMIT LIE THEORY}

for charts $\phi_{n}: P_{n} \rightarrow Q_{n} \subseteq M_{n}$. Let $\psi: V \rightarrow W \subseteq Y$ be a chart for $Y$, where $V \subseteq E$ is open. Then $U:=(f \circ \phi)^{-1}(W)$ is an open subset of $P_{\phi} \subseteq \mathbb{K}^{s}$, and $U_{n}:=U \cap P_{n}$ is open in $P_{n} \subseteq \mathbb{K}^{d_{n}}$ for each $n$. Consider $g:=\left.\psi^{-1} \circ(f \circ \phi)\right|_{U} ^{W}: U \rightarrow V$. Then $\left.g\right|_{U_{n}}=\left.\psi^{-1} \circ\left(f_{n} \circ \phi_{n}\right)\right|_{U_{n}} ^{W}: U_{n} \rightarrow V$ is $C_{\mathbb{K}}^{r}$ for each $n \in \mathbb{N}$. Hence, $g$ is $C_{\mathbb{K}}^{r}$ by Lemma 1.9(a), whence so is $f$ on the open neighbourhood $Q_{\phi}$ of $x$ and hence on all of $M$, as $x$ was arbitrary. Thus $\left(M,\left(\lambda_{n}\right)_{n \in \mathbb{N}}\right)=\underline{\lim } \mathcal{S}$ in the category of $C_{\mathbb{K}}^{r}$-manifolds, for all $r \in \mathbb{N}_{0} \cup\{\infty\}$. The uniqueness of a $C_{\mathbb{K}}^{\infty}$-manifold structure on $M$ with the described properties follows from the universal property of direct limits.

Throughout the remainder of this section, $M$ will be equipped with the $C_{\mathbb{K}}^{\infty}$-manifold structure just defined. In the proofs, we shall always reduce to the case where $I=\mathbb{N}$ and $M_{1} \subseteq M_{2} \subseteq \ldots$ (by the above argument), without further mention.

Proposition 3.2. If $\mathbb{F} \subseteq \mathbb{K}$ is a non-discrete, closed subfield, then $\left(M,\left(\lambda_{i}\right)_{i \in I}\right)=\lim _{\mathcal{S}}$ also in the category of $C_{\mathbb{F}}^{\infty}$-manifolds (e.g. $\mathbb{K}=\mathbb{C}, \mathbb{F}=\mathbb{R}$ ).

Proof. Let $\mathcal{A}$ be the $C_{\mathbb{K}}^{\infty}$-atlas of $M$ described in the proof of Theorem 3.1. Given a non-discrete closed subfield $\mathbb{F} \subseteq \mathbb{K}$, let $\mathcal{A}_{\mathbb{F}}$ be the corresponding atlas obtained when considering each $M_{i}$ merely as a $C_{\mathbb{F}}^{\infty}$-manifold over $\mathbb{F}$. Then $\mathcal{A} \subseteq \mathcal{A}_{\mathbb{F}}$, entailing that $\left(M,\left(\lambda_{n}\right)_{n \in N}\right)=\lim _{\longrightarrow} \mathcal{S}$ also in the category of $C_{\mathbb{F}}^{\infty}$-manifolds.

Proposition 3.3. Assume that $U_{i} \subseteq M_{i}$ is open and $\lambda_{i, j}\left(U_{j}\right) \subseteq U_{i}$ whenever $i \geqslant j$. Then $U:=$ $\bigcup_{i \in I} U_{i}$ is open in $M$. For the $C_{\mathbb{K}}^{\infty}$-manifold structure induced by $M$ on its open subset $U$, we have $\left(U,\left(\left.\lambda_{i}\right|_{U_{i}} ^{U}\right)_{i \in I}\right)=\lim _{\longrightarrow}\left(\left(U_{i}\right)_{i \in I},\left(\left.\lambda_{i, j}\right|_{U_{j}} ^{U_{i}}\right)_{i \geqslant j}\right)$ in the category of $C_{\mathbb{K}}^{\infty}$-manifolds.

Proof. Given open subsets $U_{n} \subseteq M_{n}$ such that $M_{1} \subseteq M_{2} \subseteq \cdots$, their union $U:=\bigcup_{n \in \mathbb{N}} U_{n}$ is open in $M$ and the topology induced by $M$ on $U$ makes $U$ the direct $\operatorname{limit} \lim _{\longrightarrow} U_{n}$ (Lemma 1.7(b)). We define an atlas $\mathcal{A}_{U}$ for $U$ turning $U$ into the direct limit of the $C_{\mathbb{K}}^{\infty}$-manifolds $U_{n}$, analogous to the definition of $\mathcal{A}$ in the proof of (a). Then $\mathcal{A}_{U} \subseteq \mathcal{A}$, whence $\left(U, \mathcal{A}_{U}\right)$ coincides with $U$, considered as an open submanifold of $M$.

Proposition 3.4. Assume that $f: X \rightarrow M$ is a $C_{\mathbb{K}}^{r}$-map, where $r \in \mathbb{N}_{0} \cup\{\infty\}$ and $X$ is a $C_{\mathbb{K}}^{r}$-manifold modelled on a metrizable topological $\mathbb{K}$-vector space $E$ (or a metrizable, locally pathconnected topological space, if $r=0$ ). Then every $x \in X$ has an open neighbourhood $S$ such that $f(S) \subseteq \lambda_{i}\left(M_{i}\right)$ for some $i \in \mathbb{N}$ and such that $\left.\lambda_{i}^{-1} \circ f\right|_{S} ^{\lambda_{i}\left(M_{i}\right)}: S \rightarrow M_{i}$ is $C_{\mathbb{K}}^{r}$.

Proof. Let $x \in X$. The assertion being local, in the case of manifolds we may assume that $X$ is an open subset of $E$. Choose a metric $d$ on $X$ defining its topology, and $k \in \mathbb{N}$ such that $f(x) \in M_{k}$. Let $\phi=\lim _{n} \phi_{n}: P \rightarrow Q$ be a chart of $M$ around $f(x)$, where $\phi_{n}$ is a chart of $M_{n}$ for all $n \geqslant k$, of the form $\phi_{n}: \Delta_{1}^{d_{n}} \rightarrow Q_{n} \subseteq M_{n}$ (see the proof of Theorem 3.1). If $f^{-1}\left(Q_{n}\right)$ is not a neighbourhood of $x$ for any $n \geqslant k$, we find $x_{n} \in f^{-1}(Q) \backslash f^{-1}\left(Q_{n}\right)$ such that $d\left(x_{n}, x\right)<2^{-n}$. Thus $x_{n} \rightarrow x$, entailing that $C:=\left\{f\left(x_{n}\right): n \in \mathbb{N}\right\} \cup\{f(x)\}$ is a compact subset of $Q$ such that $C \nsubseteq Q_{n}$ for any $n \geqslant k$. Since $Q=\lim Q_{n}$, this contradicts Lemma 1.7(d). Hence, there exists $n \geqslant k$ such that $f^{-1}\left(Q_{n}\right)$ is a neighbourhood of $x$. Let $S:=\left(f^{-1}\left(Q_{n}\right)\right)^{0}$ be its interior. Then $S \rightarrow \mathbb{K}^{s}, y \mapsto \phi^{-1}(f(y))=\phi_{n}^{-1}(f(y))$ is a $C_{\mathbb{K}^{r}}^{r}$-map taking its values in the closed vector subspace $\mathbb{K}^{d_{n}}$ of $\mathbb{K}^{s}$, whence also its co-restriction $\left.\phi_{n}^{-1} \circ f\right|_{S} ^{Q_{n}}: S \rightarrow \Delta_{1}^{d_{n}}$ is $C_{\mathbb{K}}^{r}$ [BGN04, Lemma 10.1]. As $\phi_{n}$ is a chart, this means that $\left.f\right|_{S} ^{M_{n}}$ is $C_{\mathbb{K}}^{r}$.

Proposition 3.5. If $\mathbb{K} \in\{\mathbb{R}, \mathbb{C}\}$ and $x \in M$, where $x=\lambda_{j}(y)$ say, then the connected component $C$ of $x \in M$ in $M$ is $\bigcup_{i \in I} \lambda_{i}\left(C_{i}\right) \cong \lim _{i \geqslant j} C_{i}$, where $C_{i}$ is the connected component of $\lambda_{i, j}(y)$ in $M_{i}$.

Proof. Given $x \in M_{n}$, we let $C$ and $C_{m}$ be its connected component in $M$ and $M_{m}$, respectively for $m \geqslant n$. Then $\bigcup_{m \geqslant n} C_{m} \subseteq C$. If $z \in C$, then we find a continuous curve $\gamma:[0,1] \rightarrow M$ such that $\gamma(0)=x$ and $\gamma(1)=z$. Since $[0,1]$ is compact, using Proposition 3.4 we find $m \geqslant n$ such 


\section{H. GLÖCKNER}

that $\gamma([0,1]) \subseteq M_{m}$, and such that $\left.\gamma\right|^{M_{m}}:[0,1] \rightarrow M_{m}$ is continuous. Thus $z \in C_{m}$. Hence, indeed, $C=\bigcup_{m \geqslant n} C_{m}$.

Proposition 3.6. If $\mathbb{K}$ is $\mathbb{R}$ or $\mathbb{C}$ and $M_{i}$ is paracompact for each $i \in I$, then the $C_{\mathbb{K}}^{\infty}$-manifold $M$ is also a $c_{\mathbb{K}}^{\infty}$-manifold, and $\left(M,\left(\lambda_{i}\right)_{i \in I}\right)=\lim \mathcal{S}$ in the category of $c_{\mathbb{K}}^{\infty}$-manifolds. Furthermore, $M$ is smoothly paracompact as a $C_{\mathbb{R}}^{\infty}$-manifold: For every open cover of $M$, there exists a $C_{\mathbb{R}}^{\infty}$-partition of unity subordinate to the cover.

Proof. Assume that $\mathbb{K}=\mathbb{R}$. In order that $M$ be smoothly paracompact, we only need to show that every connected component $C$ of $M$ is smoothly paracompact. Pick $c \in C$. We may assume that $c \in M_{1}$ after passing to a cofinal subsystem; we let $C_{n}$ be the connected component of $c$ in $M_{n}$ for each $n \in \mathbb{N}$. Then $\bigcup_{n \in \mathbb{N}} C_{n}$ is the connected component of $c$ in $M$ (see Proposition 3.5) and hence coincides with $C$; furthermore, $C=\underline{\lim } C_{n}$, by Proposition 3.3. After replacing $M$ with $C$ and $M_{n}$ with $C_{n}$ for each $n$, we may assume that each $M_{n}$ is a connected, paracompact finite-dimensional $C_{\mathbb{R}}^{\infty}$-manifold and hence $\sigma$-compact. This entails that $M=\bigcup_{n \in \mathbb{N}} M_{n}$ is $\sigma$-compact and therefore Lindelöf. Hence, by [KM97, Theorem 16.10], $M$ will be smoothly paracompact if we can show that $M$ is smoothly regular in the sense that, for every $x \in M$ and open neighbourhood $\Omega$ of $x$ in $M$, there exists a smooth function ('bump function') $f: M \rightarrow \mathbb{R}$ such that $f(x) \neq 0$ and $\left.f\right|_{M \backslash \Omega}=0$.

If each $\lambda_{n, m}$ is a topological embedding onto a closed submanifold, then $M$ is a regular topological space (see [Han71, Proposition 4.3(ii)]), whence smooth regularity passes from the modelling space ${ }^{2}$ to $M$ (cf. [Glo03a, Proof of Theorem 6.4]). In the fully general case to be investigated here, we do not know a priori that $M$ is regular, whence we have to prove smooth regularity of $M$ by hand. Essentially, we need to go once more through our construction of charts and build up bump functions step by step. Let $x \in M$ and $\Omega$ be an open neighbourhood of $x$ in $M$. Passing to a co-final subsequence, we may assume that $x \in M_{1}$.

Let $r_{n}:=1+2^{-n}$ for $n \in \mathbb{N}$ and $\Delta_{r_{n}}^{d_{n}}:=\Delta_{r_{n}}^{d_{n}}(\mathbb{R})$. Pick a chart $\psi_{1}: \Delta_{r_{1}}^{d_{1}} \rightarrow W_{1} \subseteq M_{1}$ of $M_{1}$ around $x$, such that $\psi_{1}(0)=x$ and such that $W_{1}$ is relatively compact in $M_{1} \cap \Omega$. Define $Q_{1}:=\psi_{1}\left(\Delta_{1}^{d_{1}}\right)$. We choose compact subsets $K_{1, j}$ of $M_{1}$ such that $W_{1} \subseteq K_{1,1}^{0} \subseteq K_{1,1} \subseteq K_{1,2}^{0} \subseteq$ $K_{1,2} \subseteq K_{1,3}^{0} \subseteq \cdots$ and $M_{1}=\bigcup_{j \in \mathbb{N}} K_{1, j}$. There exists a smooth function $h_{1}: \Delta_{r_{1}}^{d_{1}} \rightarrow \mathbb{R}$ such that $\operatorname{supp}\left(h_{1}\right) \subseteq \Delta_{1}^{d_{1}}$ and $h_{1}(0)=1$. Define $f_{1}: M_{1} \rightarrow \mathbb{R}, f_{1}(y):=0$ if $y \notin W_{1}, f_{1}(y):=h_{1}\left(\psi_{1}^{-1}(y)\right)$ if $y \in W_{1}$. Then $f_{1}$ is smooth, $\operatorname{supp}\left(f_{1}\right) \subseteq Q_{1}$, and $f_{1}(x)=1$.

The Extension Lemma 2.3 provides a chart $\psi_{2}: \Delta_{r_{2}}^{d_{2}} \rightarrow W_{2} \subseteq M_{2}$ onto an open, relatively compact subset $W_{2}$ of $M_{2} \cap \Omega$ such that $\left.\psi_{2}\right|_{\Delta_{r_{2}}^{d_{1}}}=\left.\psi_{1}\right|_{\Delta_{r_{2}}^{d_{1}}}$. We choose compact subsets $K_{2, j}$ of $M_{2}$ such that $K_{1, j} \subseteq K_{2, j}^{0}$ and $W_{2} \subseteq K_{2,1}^{0} \subseteq K_{2,1} \subseteq K_{2,2}^{0} \subseteq K_{2,2} \subseteq \cdots$ and $M_{2}=\bigcup_{j \in \mathbb{N}} K_{2, j}$. Then $K_{1,1} \backslash Q_{1}$ is a compact subset of $M_{1}$ and hence also of $M_{2}$. Therefore, $A:=\psi_{2}^{-1}\left(K_{1,1} \backslash Q_{1}\right)$ is closed in $\Delta_{r_{2}}^{d_{2}}$, and it does not meet the compact subset $\operatorname{supp}\left(h_{1}\right) \subseteq \Delta_{1}^{d_{1}} \subseteq \Delta_{r_{2}}^{d_{2}}$ (which is mapped into $Q_{1}$ by $\left.\psi_{2}\right)$. Hence, there exists $\left.\varepsilon \in\right] 0,1\left[\operatorname{such}\right.$ that $A \cap\left(\operatorname{supp}\left(h_{1}\right) \times \Delta_{\varepsilon}^{d_{2}-d_{1}}\right)=\emptyset$. We let $\xi: \mathbb{R} \rightarrow \mathbb{R}$ be a smooth function such that $\xi(0)=1$ and $\operatorname{supp}(\xi) \subseteq]-\varepsilon^{2}, \varepsilon^{2}[$. Then

$$
h_{2}: \Delta_{r_{2}}^{d_{2}} \rightarrow \mathbb{R}, \quad h_{2}(y, z):=h_{1}(y) \cdot \xi\left(\left(\|z\|_{2}\right)^{2}\right) \quad \text { for } y \in \Delta_{r_{2}}^{d_{1}}, z \in \Delta_{r_{2}}^{d_{2}-d_{1}}
$$

(where $\|\cdot\|_{2}$ is the euclidean norm on $\mathbb{R}^{d_{2}-d_{1}}$ ) is a smooth map such that $\operatorname{supp}\left(h_{2}\right) \subseteq \Delta_{1}^{d_{2}}$. Then $f_{2}(y):=0$ if $y \notin W_{2}, f_{2}(y):=h_{2}\left(\psi_{2}^{-1}(y)\right)$ for $y \in W_{2}$ defines a smooth function $f_{2}: M_{2} \rightarrow \mathbb{R}$. We have $\operatorname{supp}\left(f_{2}\right) \subseteq Q_{2}:=\psi_{2}\left(\Delta_{1}^{d_{2}}\right)$, and $\left.f_{2}\right|_{K_{1,1}}=\left.f_{1}\right|_{K_{1,1}}$, because $\left.f_{2}\right|_{Q_{1}}=\left.f_{1}\right|_{Q_{1}}$ by definition and also $\left.f_{2}\right|_{K_{1,1} \backslash Q_{1}}=0=\left.f_{1}\right|_{K_{1,1} \backslash Q_{1}}$.

Proceeding in this way, we find charts $\psi_{n}: \Delta_{r_{n}}^{d_{n}} \rightarrow W_{n} \subseteq M_{n}$ with relatively compact image $W_{n} \subseteq \Omega$, compact subsets $K_{n, j}$ of $M_{n}$ with union $M_{n}$ such that $W_{n} \subseteq K_{n, 1}, K_{n, j} \subseteq K_{n, j+1}^{0}$

\footnotetext{
${ }^{2}$ See [KM97, Theorem 16.10] or [Glo03a, Proof of Theorem 6.4] for the smooth regularity of $\mathbb{R}^{\infty}$.
} 


\section{FUndAMENTALS OF DIRECT LIMIT LIE THEORY}

and $K_{n-1, j} \subseteq K_{n, j}^{0}$ for all $n, j \in \mathbb{N}, n \geqslant 2$; and smooth maps $f_{n}: M_{n} \rightarrow \mathbb{R}$ such that $\operatorname{supp}\left(f_{n}\right) \subseteq$ $Q_{n}:=\psi_{n}\left(\Delta_{1}^{d_{n}}\right)$ and $\left.f_{n+1}\right|_{K_{n, n}}=\left.f_{n}\right|_{K_{n, n}}$ for all $n \in \mathbb{N}$, whence

$$
\left.f_{m}\right|_{K_{n, n}}=\left.f_{n}\right|_{K_{n, n}} \text { for all } n, m \in \mathbb{N} \text { such that } m \geqslant n .
$$

Let $U_{n}$ be the interior $K_{n, n}^{0}$ of $K_{n, n}$ in $M_{n}$. Then $U_{1} \subseteq U_{2} \subseteq \cdots$ and $M=\bigcup_{n \in \mathbb{N}} U_{n}$, whence $M=\lim _{n} U_{n}$ as a smooth manifold by Proposition 3.3. By (1), the smooth maps $\left.f_{n}\right|_{U_{n}}$ form a cone and hence induce a smooth map $f: M \rightarrow \mathbb{R}$, such that $\left.f\right|_{U_{n}}=\left.f_{n}\right|_{U_{n}}$ for each $n \in \mathbb{N}$. Then $f(x)=f_{1}(x)=1$. If $y \in M \backslash \Omega$, we find $n \in \mathbb{N}$ such that $y \in U_{n}$. Then $f(y)=f_{n}(y)=0$ because $\operatorname{supp}\left(f_{n}\right) \subseteq Q_{n} \subseteq W_{n} \subseteq \Omega$. Hence, $f$ is a bump function around $x$ carried by $\Omega$, as desired. Thus $M$ is smoothly paracompact.

Direct limit properties when $\mathbb{K} \in\{\mathbb{R}, \mathbb{C}\}$. Since $M$ (respectively its underlying real manifold) is smoothly regular, $M$ is smoothly Hausdorff, i.e. $C_{\mathbb{R}}^{\infty}(M, \mathbb{R})$ separates points on $M$. Let $\mathcal{A}$ be an $C_{\mathbb{K}}^{\infty}$-atlas for $M$. Being a smoothly Hausdorff $C_{\mathbb{K}}^{\infty}$-manifold modelled on a Mackey complete locally convex space, $(M, \mathcal{A})$ can be made a $c_{\mathbb{K}}^{\infty}$-manifold $\left(c^{\infty}(M), \mathcal{A}\right)$ by replacing its topology with the final topology with respect to the given charts, when the topology on the modelling space has been replaced with its $c^{\infty}$-topology. Since $c^{\infty}\left(\mathbb{K}^{s}\right)=\mathbb{K}^{s}$, the topology on $M$ remains unchanged, and thus $c^{\infty}(M)=M$. In view of Lemma 1.9(b), the desired direct limit properties can be checked as in the proof of Theorem 3.1.

Proposition 3.7. Assume that also $\mathcal{T}:=\left(\left(N_{i}\right)_{i \in I},\left(\mu_{i, j}\right)_{i \geqslant j}\right)$ is a direct system of finite-dimensional $C_{\mathbb{K}}^{\infty}$-manifolds and injective $C_{\mathbb{K}}^{\infty}$-immersions, over the same index set. Then also

$$
\mathcal{P}:=\left(\left(M_{i} \times N_{i}\right)_{i \in I},\left(\lambda_{i, j} \times \mu_{i, j}\right)_{i \geqslant j}\right)
$$

is such a direct system. Let $\left(N,\left(\mu_{i}\right)\right)=\underline{\lim } \mathcal{T}$. The $C_{\mathbb{K}}^{\infty}$-maps $\eta_{i}:=\lambda_{i} \times \mu_{i}: M_{i} \times N_{i} \rightarrow M \times N$ define a cone $\left(M \times N,\left(\eta_{i}\right)_{i \in I}\right)$ over $\mathcal{P}$, which induces a $C_{\mathbb{K}}^{\infty}$-diffeomorphism

$$
\eta: \stackrel{\lim }{\longrightarrow}\left(M_{i} \times N_{i}\right) \rightarrow\left(\lim _{\longrightarrow} M_{i}\right) \times\left(\lim _{\longrightarrow} N_{i}\right)
$$

Proof. Let $e_{n}:=\operatorname{dim}_{\mathbb{K}}\left(N_{n}\right)$ and $t:=\sup \left\{e_{n}: n \in \mathbb{N}\right\}$. The map $\zeta: \mathbb{K}^{s+t}=\underline{\lim } \mathbb{K}^{d_{n}+e_{n}} \rightarrow \mathbb{K}^{s} \times \mathbb{K}^{t}$ analogous to $\eta$ is an isomorphism of topological vector spaces [Glo03a, Proposition 3.3], [HST01, Theorem 4.1]. Let $\mathcal{A}$ be the atlas for $M=\bigcup_{n \in \mathbb{N}} M_{n}$ from the proof of Theorem 3.1; let $\mathcal{B}$ and $\mathcal{C}$ be analogous atlases for $N=\bigcup_{n \in \mathbb{N}} N_{n}$ and $P:=\bigcup_{n \in \mathbb{N}}\left(M_{n} \times N_{n}\right)$. Then $\mathcal{D}:=\{\phi \times \psi: \phi \in \mathcal{A}, \psi \in \mathcal{B}\}$ is a $C_{\mathbb{K}}^{\infty}$-atlas making $M \times N$ the direct product of $M$ and $N$ in the category of $C_{\mathbb{K}}^{\infty}$-manifolds. Since $\left\{\left.(\phi \times \psi) \circ \zeta\right|_{\zeta^{-1}\left(P_{\phi} \times P_{\psi}\right)}: \phi \in \mathcal{A}, \psi \in \mathcal{B}\right\} \subseteq \mathcal{C}$, the map $\eta=\mathrm{id}: P \rightarrow M \times N$ is a $C_{\mathbb{K}}^{\infty}$-diffeomorphism.

Proposition 3.8. If $\mathbb{K}=\mathbb{R}$, each $M_{i}$ is a finite-dimensional, real analytic manifold and each $\lambda_{i, j}$ an injective, real analytic immersion, then there exists a $c_{\mathbb{R}}^{\omega}$-manifold structure on $M$ such that $M=\lim \mathcal{S}$ in the category of $c_{\mathbb{R}}^{\omega}$-manifolds (and $c_{\mathbb{R}}^{\omega}$-maps), and which is compatible with the above $C_{\mathbb{R}}^{\infty}$-manifold structure on $M$. Analogues of Propositions 3.3, 3.4 and 3.7 hold for the $c_{\mathbb{R}}^{\omega}$-manifold structures (and $c_{\mathbb{R}}^{\omega}$-maps).

Proof. Using the $C_{\mathbb{R}}^{\omega}$-case of the Extension Lemma 2.3, the construction described in the proof of Theorem 3.1 provides a subatlas $\mathcal{B}$ of the $C_{\mathbb{R}}^{\infty}$-atlas $\mathcal{A}$ consisting of charts $\phi=\underline{\lim } \phi_{n}$ where each $\phi_{n}$ is a $C_{\mathbb{R}}^{\omega}$-diffeomorphism. Using Lemma $1.9(\mathrm{~b})$, the above arguments show that the chart changes for charts in $\mathcal{B}$ are $c_{\mathbb{R}}^{\omega}$, whence indeed $M$ has a compatible $c_{\mathbb{R}}^{\omega}$-manifold structure. Similarly, Lemma 1.9(b) entails the validity of the $c_{\mathbb{R}}^{\omega}$-analogues of Propositions 3.3 and 3.7. The proof of Proposition 3.4 carries over directly.

Remark 3.9. As a consequence of Proposition 3.4 (and Proposition 3.5), for the singular homology groups of $M$ over a commutative ring $R$ we have $\left(H_{k}(M),\left(H_{k}\left(\lambda_{i}\right)\right)_{i \in I}\right)=\underline{\lim }\left(H_{k}\left(M_{i}\right),\left(H_{k}\left(\lambda_{i, j}\right)_{i \geqslant j}\right)\right)$ 


\section{H. GLÖCKNER}

for all $k \in \mathbb{N}_{0}$. Likewise, given $\ell \in I$ and $x \in M_{\ell}$, for the homotopy groups we have $\pi_{k}\left(M, \lambda_{\ell}(x)\right)=\lim _{k} \pi_{k}\left(M_{i}, \lambda_{i, \ell}(x)\right)$.

\section{Direct limits of finite-dimensional Lie groups}

Let $\mathbb{K}$ be $\mathbb{R}, \mathbb{C}$ or a local field, and $\mathcal{S}:=\left(\left(G_{i}\right)_{i \in I},\left(\lambda_{i, j}\right)_{i \geqslant j}\right)$ be a countable direct system of finitedimensional $C_{\mathbb{K}}^{\infty}$-Lie groups $G_{i}$ and $C_{\mathbb{K}}^{\infty}$-homomorphisms $\lambda_{i, j}: G_{j} \rightarrow G_{i}$; if $\operatorname{char}(\mathbb{K})>0$, we assume that each $\lambda_{i, j}$ is an injective immersion. In this section, we construct a direct limit Lie group for $\mathcal{S}$, and discuss some of its properties.

Remark 4.1. (a) As in the classical real and complex cases, every finite-dimensional $C_{\mathbb{K}}^{\infty}$-Lie group over a local field $\mathbb{K}$ of characteristic 0 admits a $C_{\mathbb{K}}^{\infty}$-compatible analytic Lie group structure, and every $C_{\mathbb{K}}^{\infty}$-homomorphism between such groups is $\mathbb{K}$-analytic [Glo03c]. However, for every local field $\mathbb{K}$ of positive characteristic, there exists a one-dimensional $C_{\mathbb{K}}^{\infty}$-Lie group which does not admit a finite-dimensional $\mathbb{K}$-analytic Lie group structure compatible with the given topological group structure [Glo05]. Thus, smooth Lie groups are properly more general than analytic Lie groups here.

(b) Consider the field $\mathbb{K}:=\mathbb{F}_{2}((X))$ of formal Laurent series over $\mathbb{F}_{2}:=\mathbb{Z} / 2 \mathbb{Z}$, and its open compact subring $\mathbb{F}_{2}[[X]]$ of formal power series. Then the squaring map $\sigma: \mathbb{F}_{2}[[X]]^{\times} \rightarrow \mathbb{F}_{2}[[X]]^{\times}$, $\sigma(x):=x^{2}$ is a $\mathbb{K}$-analytic (and hence smooth) homomorphism which is injective (since $\mathbb{F}_{2}[[X]]^{\times}$ is isomorphic to the power $\left(\mathbb{Z}_{2}\right)^{\mathbb{N}}$ of the group of 2-adic integers as a group by [Wei67, ch. II-3, Proposition 10], and thus torsion-free) but not an immersion because $f^{\prime}(x)=2 \mathrm{id}=0$ for all $x \in \mathbb{F}_{2}[[X]]^{\times}$. This explains that an extra hypothesis is needed in positive characteristic. ${ }^{3}$

\section{Associated injective quotient system}

If $\mathbb{K}=\mathbb{R}$ (or if $\operatorname{char}(\mathbb{K})>0$, in which case we obtain trivial subgroups), we let $N_{j}:=\overline{\bigcup_{i \geqslant j} \operatorname{ker} \lambda_{i, j}}$ for $j \in I$. If $\mathbb{K}$ is a local field of characteristic 0 , we let $N_{j}=\bigcup_{i \geqslant j} \operatorname{ker} \lambda_{i, j}$ for $j \in I$ and note that $N_{j}$ is locally closed and hence closed in $G_{j}$, because $G_{j}$ has an open compact subgroup $U$ which satisfies an ascending chain condition on closed subgroups. If $\mathbb{K}=\mathbb{C}$, we let $N_{j} \subseteq G_{j}$ be the intersection of all closed complex Lie subgroups $S$ of $G_{j}$ such that $\bigcup_{i \geqslant j}$ ker $\lambda_{i, j} \subseteq S$. Then $N_{j}$ is a complex Lie subgroup of $G_{j}$ (as $G_{j}$ satisfies a descending chain condition on closed, connected subgroups), and thus $N_{j}$ is the smallest closed, complex Lie subgroup of $G_{j}$ containing $\bigcup_{i \geqslant j} \operatorname{ker} \lambda_{i, j}$. By minimality, $N_{j}$ is invariant under inner automorphisms and hence a normal subgroup of $G_{j}$.

Then, in either case, there is a uniquely determined $\mathbb{K}$-Lie group structure on $\bar{G}_{j}:=G_{j} / N_{j}$ which makes the canonical quotient homomorphism $q_{j}: G_{j} \rightarrow \bar{G}_{j}$ a submersion. Each $\lambda_{i, j}$ factors to a $C_{\mathbb{K}}^{\infty}$-homomorphism $\bar{\lambda}_{i, j}: \bar{G}_{j} \rightarrow \bar{G}_{i}$, uniquely determined by $\bar{\lambda}_{i, j} \circ q_{j}=q_{i} \circ \lambda_{i, j}$. Then $\overline{\mathcal{S}}=\left(\left(\bar{G}_{i}\right)_{i \in I},\left(\bar{\lambda}_{i, j}\right)_{i \geqslant j}\right)$ is a direct system of finite-dimensional $C_{\mathbb{K}}^{\infty}$-Lie groups and injective $C_{\mathbb{K}}^{\infty}$-homomorphisms $\bar{\lambda}_{i, j}: \bar{G}_{j} \rightarrow \bar{G}_{i}$; it is called the injective quotient system associated with $\mathcal{S}$ (cf. [NRW91]). Each $\bar{\lambda}_{i, j}$ is an injective immersion of class $C_{\mathbb{K}}^{\infty}$.

Remark 4.2. The example $\mathbb{C}^{\times} \stackrel{\sigma}{\rightarrow} \mathbb{C}^{\times} \stackrel{\sigma}{\rightarrow} \cdots$ with the squaring map $\sigma: \mathbb{C}^{\times} \rightarrow \mathbb{C}^{\times}, \sigma(z):=z^{2}$ shows that $\overline{\bigcup_{i \geqslant j} \operatorname{ker} \lambda_{i, j}}$ need not be a complex Lie subgroup of $G_{j}$.

TheOREM 4.3. For $\mathcal{S}$ and $\overline{\mathcal{S}}$ as before, the following hold.

(a) A direct limit $\left(G,\left(\bar{\lambda}_{i}\right)_{i \in I}\right)$ for $\overline{\mathcal{S}}$ exists in the category of $C_{\mathbb{K}}^{\infty}$-Lie groups modelled on topological $\mathbb{K}$-vector spaces; $G$ is modelled on the locally convex topological $\mathbb{K}$-vector space $\mathbb{K}^{s}$,

${ }^{3}$ Slightly more generally, to establish Theorem 4.3 for $\operatorname{char}(\mathbb{K})>0$, it would be enough to assume that $G_{j} / N_{j}$ admits a $C_{\mathbb{K}}^{\infty}$-Lie group structure for each $j \in I$ which makes the quotient map $G_{j} \rightarrow G_{j} / N_{j}$ a submersion, where $N_{j}:=\overline{\bigcup_{i \geqslant j} \operatorname{ker} \lambda_{i, j}}$, and that the induced homomorphisms $G_{j} / N_{j} \rightarrow G_{i} / N_{i}$ be immersions, for all $i \geqslant j$. 


\section{FUndAMENTALS OF DIRECT LIMIT LIE THEORY}

where $s:=\sup \left\{\operatorname{dim}_{\mathbb{K}} \bar{G}_{i}: i \in I\right\} \in \mathbb{N}_{0} \cup\{\infty\}$. Forgetting the $\mathbb{K}$-Lie group structure, we also have $\left(G,\left(\bar{\lambda}_{i}\right)_{i \in I}\right)=\underline{\lim } \overline{\mathcal{S}}$ in the categories of sets, abstract groups, topological spaces, topological groups, the category of $C_{\mathbb{K}}^{\infty}$-manifolds modelled on topological $\mathbb{K}$-vector spaces, and the category of $C_{\mathbb{F}}^{\infty}$-Lie groups modelled on topological $\mathbb{F}$-vector spaces, for every non-discrete closed subfield $\mathbb{F}$ of $\mathbb{K}$. Furthermore, $L\left(\bar{\lambda}_{i}\right): L\left(\bar{G}_{i}\right) \rightarrow L(G)$ is injective for each $i \in I$, and

$$
\left(L(G),\left(L\left(\bar{\lambda}_{i}\right)\right)_{i \in I}\right)=\underline{\lim }\left(\left(L\left(\bar{G}_{i}\right)\right)_{i \in I},\left(L\left(\bar{\lambda}_{i, j}\right)\right)_{i \geqslant j}\right)
$$

in the category of locally convex $\mathbb{K}$-vector spaces (and in the categories of sets, $\mathbb{K}$-Lie algebras, topological spaces, topological $\mathbb{K}$-Lie algebras, topological $\mathbb{K}$-vector spaces, and $C_{\mathbb{K}}^{\infty}$-manifolds; also in the category of $c_{\mathbb{K}}^{\omega}$-manifolds, if $\left.\mathbb{K} \in\{\mathbb{R}, \mathbb{C}\}\right)$.

(b) Set $\lambda_{i}:=\bar{\lambda}_{i} \circ q_{i}$ for $i \in I$. If $\mathbb{K} \neq \mathbb{C}$ or if $\mathbb{K}=\mathbb{C}$ and $N_{j}=\overline{\bigcup_{i \geqslant j} \operatorname{ker} \lambda_{i, j}}$ for each $j \in I$, then $\left(G,\left(\lambda_{i}\right)_{i \in I}\right)=\lim \mathcal{S}$ in the category of $C_{\mathbb{K}}^{\infty}$-Lie groups modelled on topological $\mathbb{K}$-vector spaces, and also in the categories of smooth manifolds modelled on topological $\mathbb{K}$-vector spaces, Hausdorff topological spaces, and (Hausdorff) topological groups.

(c) If $\mathbb{K}=\mathbb{C}$, then $\left(G,\left(\lambda_{i}\right)_{i \in I}\right)=\lim \mathcal{S}$ in the category of complex Lie groups modelled on complex locally convex spaces.

(d) If $\mathbb{K}=\mathbb{R}$, then $G$ is a $c_{\mathbb{R}}^{\infty}$-regular $c_{\mathbb{R}}^{\infty}$-Lie group which is smoothly paracompact. Furthermore, $\left(G,\left(\lambda_{i}\right)_{i \in I}\right)=\underline{\lim } \mathcal{S}$ in the category of $c_{\mathbb{R}}^{\infty}$-Lie groups, and in the category of $c_{\mathbb{R}}^{\infty}$-manifolds.

(e) If $\mathbb{K}=\mathbb{C}$, then $G$ is a $c_{\mathbb{C}}^{\infty}$-regular, $c_{\mathbb{C}}^{\infty}$-Lie group such that $\left(G,\left(\lambda_{i}\right)_{i \in I}\right)=\underline{\lim } \mathcal{S}$ in the category of $c_{\mathbb{C}}^{\infty}$-Lie groups and $\left(G,\left(\lambda_{i}\right)_{i \in I}\right)=\underline{\lim } \overline{\mathcal{S}}$ in the category of $c_{\mathbb{C}}^{\infty}$-manifolds.

(f) If $\mathbb{K}=\mathbb{R}$, then there exists a $c_{\mathbb{R}}^{\omega}$-regular, $c_{\mathbb{R}}^{\omega}$-Lie group structure on $G$, compatible with the $C_{\mathbb{R}}^{\infty}$-Lie group structure from (a), such that $\left(G,\left(\lambda_{i}\right)_{i \in I}\right)=\lim \mathcal{S}$ in the category of $c_{\mathbb{R}}^{\omega}$-Lie groups. For the underlying $c_{\mathbb{R}^{-}}^{\omega}$ manifold, we have $\left(G,\left(\lambda_{i}\right)_{i \in I}\right) \vec{\longrightarrow} \underline{\lim } \mathcal{S}$ in the category of such manifolds.

Proof. (a) Let $\left(G,\left(\bar{\lambda}_{i}\right)_{i \in I}\right)$ be a direct limit for $\overline{\mathcal{S}}$ in the category of topological groups; then $\left(G,\left(\bar{\lambda}_{i}\right)_{i \in I}\right)=\lim \overline{\mathcal{S}}$ also in the categories of sets, groups, and topological spaces [TSH98, Theorem 2.7]. Thus, Theorem 3.1 provides a $C_{\mathbb{K}}^{\infty}$-manifold structure on $G$ making $\left(G,\left(\bar{\lambda}_{i}\right)_{i \in I}\right)$ a direct limit of $\overline{\mathcal{S}}$ in the category of $C_{\mathbb{K}}^{\infty}$-manifolds modelled on topological $\mathbb{K}$-vector spaces, and also in the category of $C_{\mathbb{F}}^{\infty}$-manifolds, for every non-discrete closed subfield $\mathbb{F} \subseteq \mathbb{K}$ (Proposition 3.2). Let $\theta: G \rightarrow G, g \mapsto g^{-1}$ and $\theta_{i}: \bar{G}_{i} \rightarrow \bar{G}_{i}$ be the inversion maps; let $\mu: G \times G \rightarrow G$ and $\mu_{i}: \bar{G}_{i} \times \bar{G}_{i} \rightarrow \bar{G}_{i}$ be multiplication. Then $\theta:=\underline{\lim } \theta_{i}$ is $C_{\mathbb{K}}^{\infty}$, as $G=\underline{\lim } \bar{G}_{i}$ in the category of $C_{\mathbb{K}}^{\infty}$-manifolds. Likewise, $\mu=\underline{\lim } \mu_{i}$ is $C_{\mathbb{K}}^{\infty}$ on $\underline{\lim }\left(\bar{G}_{i} \times \vec{G}_{i}\right)$ and hence on $\overrightarrow{G \times} G$, in view of Proposition 3.7. Hence, $G$ is a $C_{\mathbb{K}}^{\infty}$-Lie group. Every cone $\left(H,\left(f_{i}\right)_{i \in I}\right)$ of $C_{\mathbb{K}}^{\infty}$-homomorphisms $f_{i}: \bar{G}_{i} \rightarrow H$ to a $C_{\mathbb{K}}^{\infty}$-Lie group $H$ uniquely determines a map $f: G \rightarrow H$ such that $f \circ \bar{\lambda}_{i}=f_{i}$ for all $i$; then $f$ is a homomorphism since $G=\underline{\lim } \bar{G}_{i}$ as a group, and $f$ is $C_{\mathbb{K}}^{\infty}$ since $G=\underline{\lim } \bar{G}_{i}$ as a $C_{\mathbb{K}}^{\infty}$-manifold. Thus $G=\underline{\lim } \bar{G}_{i}$ as a $C_{\mathbb{K}}^{\infty}$-Lie group (and, likewise, as a $C_{\mathbb{F}}^{\infty}$-Lie group).

Determination of the Lie algebra of $L(G) \cdot \mathcal{T}:=\left(\left(L\left(\bar{G}_{i}\right)\right)_{i \in I},\left(L\left(\bar{\lambda}_{i, j}\right)\right)_{i \geqslant j}\right)$ is a countable, strict direct system of Lie algebras, since $L\left(\bar{\lambda}_{i, j}\right)$ is injective for all $i \geqslant j$. We recall from [Glo03a, HST01] that $\mathcal{T}$ has a direct limit $\left(\mathfrak{g},\left(\phi_{i}\right)_{i \in I}\right)$ in the category of topological $\mathbb{K}$-Lie algebras; here $\mathfrak{g}$ carries the finite topology (see $\S 1.8$ ), each $\phi_{i}$ is injective, and $\left(\mathfrak{g},\left(\phi_{i}\right)_{i \in I}\right)=\lim \mathcal{T}$ also holds in the categories of sets, $\mathbb{K}$-Lie algebras, topological spaces, topological $\mathbb{K}$-vector spaces, and locally convex topological $\mathbb{K}$-vector spaces. By Lemma 1.9(a), furthermore $\left(\mathfrak{g},\left(\phi_{i}\right)_{i \in I}\right)=\underline{\lim } \mathcal{T}$ in the category of $C_{\mathbb{K}}^{\infty}$-manifolds and $C_{\mathbb{K}}^{\infty}$-maps (and also in the category of $c_{\mathbb{K}}^{\omega}$-manifolds and $\overrightarrow{c_{\mathbb{K}}^{\omega}}$-maps by Lemma $1.9(\mathrm{~b})$, if $\mathbb{K}=\mathbb{R}$ or $\mathbb{C})$. The mappings $L\left(\bar{\lambda}_{i}\right): L\left(\bar{G}_{i}\right) \rightarrow L(G)$ form a cone over $\mathcal{T}$ and hence induce a continuous Lie algebra homomorphism $\Lambda: \mathfrak{g}=\lim _{\longrightarrow} L\left(\bar{G}_{i}\right) \rightarrow L(G)$, determined by $\Lambda \circ \phi_{i}=L\left(\bar{\lambda}_{i}\right)$. To see that $\Lambda$ is surjective, let a geometric tangent vector $v \in L(G)=T_{1} G$ be given, say $v=[\gamma]$ where $\gamma: U \rightarrow G$ is a smooth map on an open zero-neighbourhood $U \subseteq \mathbb{K}$, such that $\gamma(0)=1$. By Proposition 3.4, 


\section{H. GLÖCKNER}

after shrinking $U$ we may assume that $\gamma(U) \subseteq \bar{\lambda}_{i}\left(\bar{G}_{i}\right)$ for some $n \in \mathbb{N}$, and that $\gamma=\bar{\lambda}_{i} \circ \eta$ for some smooth map $\eta: U \rightarrow \bar{G}_{i}$. Then $\Lambda\left(\phi_{i}([\eta])\right)=L\left(\bar{\lambda}_{i}\right)([\eta])=\left[\bar{\lambda}_{i} \circ \eta\right]=[\gamma]=v$, as desired. Because $\mathfrak{g}=\bigcup_{i \in I} \operatorname{im} \phi_{i}$ and $\Lambda \circ \phi_{i}=L\left(\bar{\lambda}_{i}\right)$, injectivity of $\Lambda$ follows from the injectivity of the maps $L\left(\bar{\lambda}_{i}\right)=T_{1}\left(\bar{\lambda}_{i}\right)$ established in Theorem 3.1. By the preceding, $\Lambda$ is an isomorphism of Lie algebras; as both $\mathfrak{g}$ and $L(G) \cong \mathbb{K}^{s}$ are equipped with the finite topology, $\Lambda$ also is an isomorphism of topological vector spaces. Hence $L(G) \cong \mathfrak{g}=\lim L\left(\bar{G}_{i}\right)$ naturally. The desired direct limit properties carry over from $\mathfrak{g}$ to $L(G)$.

(b) and (c) Assume that $H$ is a $C_{\mathbb{K}}^{\infty}$-Lie group modelled on a topological $\mathbb{K}$-vector space and $\left(f_{i}\right)_{i \in I}$ a family of $C_{\mathbb{K}}^{\infty}$-homomorphisms $f_{i}: G_{i} \rightarrow H$ such that $\left(H,\left(f_{i}\right)_{i \in I}\right)$ is a cone over $\mathcal{S}$. Given $j \in I$, for any $i \geqslant j$ we then have $f_{j}=f_{i} \circ \lambda_{i, j}$ and thus ker $\lambda_{i, j} \subseteq \operatorname{ker} f_{j}$, entailing that $\overline{\bigcup_{i \geqslant j} \operatorname{ker} \lambda_{i, j}} \subseteq \operatorname{ker} f_{j}$. In the situation of (b), this means that $N_{j} \subseteq \operatorname{ker} f_{j}$. In the situation of (c), we assume that $H$ is modelled on a complex locally convex space. Then $\operatorname{ker} f_{j}$ is a complex Lie subgroup of $G_{j}$ (Lemma 4.4), which contains $\bigcup_{i \geqslant j} \operatorname{ker} \lambda_{i, j}$; hence also $N_{j} \subseteq \operatorname{ker} f_{j}$ in (c). In any case, we deduce that $f_{j}=\bar{f}_{j} \circ q_{j}$ for a homomorphism $\bar{f}_{j}: \bar{G}_{j} \rightarrow H$, which is $C_{\mathbb{K}}^{\infty}$ because $q_{j}$ admits smooth local sections. Then $\left(\left(\bar{f}_{i}\right)_{i \in I}, H\right)$ is a cone over $\overline{\mathcal{S}}$ and hence induces a unique $C_{\mathbb{K}}^{\infty}$ homomorphism $f: G \rightarrow H$ such that $f \circ \bar{\lambda}_{i}=\bar{f}_{i}$, since $\left(G,\left(\bar{\lambda}_{i}\right)_{i \in I}\right)=\underline{\lim } \overline{\mathcal{S}}$ in the category of $C_{\mathbb{K}}^{\infty}$-Lie groups. Then $f \circ \lambda_{i}=f \circ \bar{\lambda}_{i} \circ q_{i}=\bar{f}_{i} \circ q_{i}=f_{i}$ for each $i \in I$, and clearly $f$ is determined by this property. Thus $\left(G,\left(\lambda_{i}\right)_{i \in I}\right)=\lim \mathcal{S}$ in the category of $C_{\mathbb{K}}^{\infty}$-Lie groups. In the situation of (b), the universal property of direct limit in the other categories of interest can be proved by trivial adaptations of the argument just given.

(d) To establish the first assertion, we may assume that $I=\mathbb{N}$, and after replacing $\mathcal{S}$ by a system equivalent to $\overline{\mathcal{S}}$ we may assume that $G_{1} \subseteq G_{2} \subseteq \cdots$, each $\lambda_{n, m}$ being the respective inclusion map. Then $L(G)=\bigcup_{n \in \mathbb{N}} L\left(G_{n}\right)$. If $\gamma: \mathbb{R} \rightarrow L(G)$ is a smooth curve, then for each $k \in \mathbb{Z}$, there exists $n_{k} \in \mathbb{N}$ such that the relatively compact set $\gamma(] k-1, k+2[)$ is contained in $L\left(G_{n_{k}}\right)$. The finitedimensional Lie group $G_{n_{k}}$ being $c_{\mathbb{R}}^{\infty}$-regular, we find a smooth curve $\left.\eta_{k}:\right] k-1, k+2\left[\rightarrow G_{n_{k}}\right.$ such that $\eta_{k}(k)=1$ and $\delta^{r}\left(\eta_{k}\right)=\gamma_{k}$. We define $\eta(t):=\eta_{k}(t) \eta_{k-1}(k) \cdots \eta_{1}(2) \eta_{0}(1)$ for $t \in[k, k+1]$ with $k \geqslant 0$, and $\eta(t):=\eta_{k}(t) \eta_{k}(k+1)^{-1} \cdots \eta_{-2}(-1)^{-1} \eta_{-1}(0)^{-1}$ for $t \in[k, k+1]$ with $k<0$. Then $\eta: \mathbb{R} \rightarrow G$ is a smooth curve such that $\eta(0)=1$ and $\delta^{r}(\eta)=\gamma$. Thus, every $\gamma \in C^{\infty}(\mathbb{R}, L(G))$ has a right product integral $\operatorname{Evol}_{G}^{r}(\gamma):=\eta \in C^{\infty}(\mathbb{R}, G)$. We define

$$
\operatorname{evol}_{G}^{r}: C^{\infty}(\mathbb{R}, L(G)) \rightarrow G, \operatorname{evol}_{G}^{r}(\gamma):=\operatorname{Evol}_{G}^{r}(\gamma)(1) .
$$

To see that $\operatorname{evol}_{G}^{r}$ is $c_{\mathbb{R}}^{\infty}$, let $\sigma: \mathbb{R} \rightarrow C^{\infty}(\mathbb{R}, L(G))$ be a smooth curve. Given $t_{0} \in \mathbb{R}$, let $U \subseteq \mathbb{R}$ be a relatively compact neighbourhood of $t_{0}$. We show that $\operatorname{evol}_{G}^{r} \circ \sigma: \mathbb{R} \rightarrow G$ is smooth on $U$. The evaluation map $C^{\infty}(\mathbb{R}, L(G)) \times \mathbb{R} \rightarrow L(G),(\gamma, t) \mapsto \gamma(t)$ being continuous (cf. [Eng89, Theorem 3.4.3 and Proposition 2.6.11]), $\sigma(U)([-1,2])$ is a compact subset of $L(G)$ and hence contained in $L\left(G_{n}\right)$ for some $n \in \mathbb{N}$, by Lemma $1.7(\mathrm{~d})$. We now consider

$$
\tau: U \rightarrow C^{\infty}(]-1,2\left[, L\left(G_{n}\right)\right), \quad \tau(t):=\left.\sigma(t)\right|_{]-1,2[} ^{L\left(G_{n}\right)} .
$$

Then $\tau$ is smooth, because the restriction map $C^{\infty}(\mathbb{R}, L(G)) \rightarrow C^{\infty}(]-1,2[, L(G))$ is continuous linear, and $C^{\infty}(]-1,2\left[, L\left(G_{n}\right)\right)$ is a closed vector subspace of $C^{\infty}(]-1,2[, L(G))$. The group $G_{n}$ being regular, evol $G_{n}: C^{\infty}(]-1,2\left[, L\left(G_{n}\right)\right) \rightarrow G_{n}$ is smooth. Since $\left.\operatorname{evol}_{G}^{r} \circ \sigma\right|_{U}=\operatorname{evol}_{G_{n}}^{r} \circ \tau$ apparently, we deduce that $\left.\operatorname{evol}_{G}^{r} \circ \sigma\right|_{U}$ is smooth. Thus, $\operatorname{evol}_{G}^{r}$ is $c_{\mathbb{R}}^{\infty}$. The desired direct limit properties can be proved as in (a) and (b), based on Proposition 3.6.

(e) As a consequence of Proposition 3.6, the $C_{\mathbb{C}}^{\infty}$-Lie group $G$ is also a $c_{\mathbb{C}}^{\infty}$-Lie group. It is $c_{\mathbb{C}}^{\infty}$-regular because its underlying $c_{\mathbb{R}}^{\infty}$-Lie group is $c_{\mathbb{R}}^{\infty}$-regular by $(\mathrm{d})$. The desired direct limit property can be proved as in (a) and (b).

(f) Using the $c_{\mathbb{R}^{-}}^{\omega}$ analogue of Proposition 3.7 (see Proposition 3.8), we see as in the proof of (a) that the $C_{\mathbb{R}}^{\infty}$-compatible $c_{\mathbb{R}}^{\omega}$-manifold structure on $G$ from Proposition 3.8 turns $G$ into 


\section{FUndAMENTALS OF DIRECT LIMIT LIE THEORY}

a $c_{\mathbb{R}}^{\omega}$-Lie group. By $(\mathrm{d})$, the latter is $c_{\mathbb{R}}^{\infty}$-regular. To see that it is $c_{\mathbb{R}}^{\omega}$-regular, let $\gamma: \mathbb{R} \rightarrow L(G)$ be a $c_{\mathbb{R}}^{\omega}$-curve and $\eta:=\operatorname{Evol}_{G}^{r}(\gamma)$ be its right product integral. Using Proposition 3.4 and its $c_{\mathbb{R}}^{\omega}$-analogue (Proposition 3.8), for each $k \in \mathbb{N}$ we find $n \in \mathbb{N}$ such that $\gamma([-k, k]) \subseteq L\left(G_{n}\right), \eta([-k, k]) \subseteq G_{n}$, and such that $\sigma:=\left.\gamma\right|_{-k, k[} ^{L\left(G_{n}\right)}$ is $c_{\mathbb{R}}^{\omega}$ and $\tau:=\left.\eta\right|_{-k, k[} ^{G_{n}}$ smooth. The finite-dimensional Lie group $G_{n}$ being $c_{\mathbb{R}}^{\omega}$-regular, the product integral $\tau$ of the $c_{\mathbb{R}}^{\omega}$-curve $\sigma$ has to be $c_{\mathbb{R}}^{\omega}$. Hence, $\left.\eta\right|_{]-k, k[}$ is $c_{\mathbb{R}}^{\omega}$ for each $k \in \mathbb{N}$ and thus $\eta$ is $c_{\mathbb{R}}^{\omega}$. Hence, $G$ is $c_{\mathbb{R}}^{\omega}$-regular. The direct limit property can be established as in (b).

We needed to assume local convexity in Theorem 4.3(c) because the proof of the following simple lemma depends on local convexity.

Lemma 4.4. Let $\phi: G \rightarrow H$ be a $C_{\mathbb{C}}^{\infty}$-homomorphism from a finite-dimensional complex Lie group to a complex Lie group modelled on a locally convex complex topological vector space. Then $K:=\operatorname{ker} \phi$ is a complex Lie subgroup of $G$. The same conclusion holds if $H$ is a $c_{\mathbb{C}}^{\omega}$-Lie group and $\phi$ a $c_{\mathbb{C}}^{\omega}$-homomorphism.

Proof. Being a closed subgroup of $G, K$ is a real Lie subgroup, with Lie algebra

$$
\mathfrak{k}=\left\{X \in L(G): \exp _{G}(\mathbb{R} X) \subseteq K\right\}=\left\{X \in L(G): \phi\left(\exp _{G}(\mathbb{R} X)\right)=\{1\}\right\} .
$$

Given $X \in \mathfrak{k}$, the map $f: \mathbb{C} \rightarrow H, f(z):=\phi\left(\exp _{G}(z X)\right)$ is complex analytic and $\left.f\right|_{\mathbb{R}}=1$, whence $f=1$ by the Identity Theorem. Hence $\mathbb{C} X \subseteq \mathfrak{k}$, whence $\mathfrak{k}$ is a complex Lie subalgebra of $L(G)$. Therefore $K$ is a complex Lie subgroup [Bou89, ch. III, $\S 4.2$, Corollary 2].

Remark 4.5. (a) In the situation of Remark 4.2, the direct system $\left(\left(\mathbb{C}^{\times}\right)_{n \in \mathbb{N}},(\sigma)_{n \geqslant m}\right)$ has the direct limit $\mathbb{R}$ in the category of real Lie groups, whereas its direct limit in the category of complex Lie groups is the trivial group. Hence, the conclusions of Theorem 4.3(a) become false in general if we replace the injective quotient system $\overline{\mathcal{S}}$ by a non-injective system $\mathcal{S}$. Also note that $\lim _{\longrightarrow} L\left(\mathbb{C}^{\times}\right)=$ $\mathbb{C} \neq\{0\}=L(\{1\})=L\left(\underline{\lim } \mathbb{C}^{\times}\right)$here.

(b) If each $\lambda_{i, j}$ is injective, then the direct systems $\mathcal{S}$ and $\overline{\mathcal{S}}$ are equivalent, whence Theorem 4.3(a) remains valid when $\overline{\mathcal{S}}$ is replaced with $\mathcal{S}$ and all bars are omitted.

Proposition 4.6. Assume that $\left(\left(G_{i}\right)_{i \in I},\left(\lambda_{i, j}\right)_{i \geqslant j}\right)$ is a countable direct system of finite-dimensional Lie groups over $\mathbb{K} \in\{\mathbb{R}, \mathbb{C}\}$ and injective $C_{\mathbb{K}}^{\infty}$-homomorphisms, with direct limit $\left(G,\left(\lambda_{i}\right)_{i \in I}\right)$. Then the following holds.

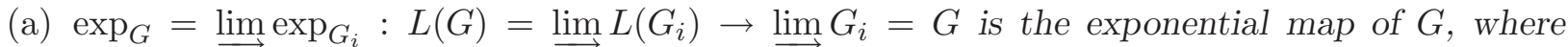
$\left.\left(L(G),\left(\overrightarrow{L\left(\lambda_{i}\right.}\right)\right)_{i \in I}\right)=\underline{\lim }\left(\left(L\left(G_{i}\right)\right),\left(L\left(\lambda_{i, j}\right)\right)\right) . \overrightarrow{T h} \operatorname{map} \exp _{G}$ is $c_{\mathbb{K}}^{\omega}$.

(b) The Trotter Product Formula $\exp _{G}(x+y)=\lim _{n \rightarrow \infty}\left(\exp _{G}((1 / n) x) \exp _{G}((1 / n) y)\right)^{n}$ holds and the Commutator Formula $\exp _{G}([x, y])=\lim _{n \rightarrow \infty}\left(\exp _{G}((1 / n) x) \exp _{G}((1 / n) y) \exp _{G}(-(1 / n) x)\right.$ $\left.\exp _{G}(-(1 / n) y)\right)^{n^{2}}$, for all $x, y \in L(G)$.

(c) Let $\left(H,\left(\mu_{i}\right)\right)=\lim _{\longrightarrow} \mathcal{T}$ for a direct system $\mathcal{T}=\left(\left(H_{i}\right),\left(\mu_{i, j}\right)\right)$ of finite-dimensional $\mathbb{K}$-Lie groups and injective $C_{\mathbb{K}}^{\infty}$-homomorphisms, and assume that $f_{i}: G_{i} \rightarrow H_{i}$ are $C_{\mathbb{K}}^{\infty}$-homomorphisms. Then $L\left(\lim _{\longrightarrow} f_{i}\right)=\lim _{\longrightarrow} L\left(f_{i}\right)$. Furthermore, every continuous homomorphism $G \rightarrow H$ is $c_{\mathbb{R}}^{\omega}$.

Proof. (a) By Theorem 4.3(a), $L(G)=\underline{\lim } L\left(G_{i}\right)$ as a $c_{\mathbb{K}}^{\omega}$-manifold. The family $\left(\exp _{G_{i}}\right)_{i \in I}$ of $c_{\mathbb{K}}^{\omega}$-maps being compatible with the direct systems by naturality of exp, there is a unique $c_{\mathbb{K}^{-}}^{\omega}$ map $\exp _{G}:=$ $\stackrel{\lim }{\longrightarrow} \exp _{G_{i}}$ such that $\exp _{G} \circ L\left(\lambda_{i}\right)=\lambda_{i} \circ \exp _{G_{i}}$ for each $i$. Given $y \in L(G)$, there are $j \in I$ and $\overrightarrow{x \in L}\left(G_{j}\right)$ such that $y=L\left(\lambda_{j}\right)(x)$. Then $\xi: \mathbb{R} \rightarrow G, \xi(t):=\exp _{G}(t x)=\lambda_{j}\left(\exp _{G_{j}}(t y)\right)$ is a smooth homomorphism such that $\xi^{\prime}(0)=L\left(\lambda_{j}\right)\left(\exp _{G_{j}}^{\prime}(0) \cdot y\right)=L\left(\lambda_{j}\right)(y)=x$. Hence, $G$ admits an exponential map (in the sense of [KM97, Definition 36.8]), and it is given by $\exp _{G}$ from above and hence $c_{\mathbb{K}}^{\omega}$.

(b) Using (a), the assertions directly follow from the finite-dimensional case. 


\section{H. GLÖCKNER}

(c) By Theorem 4.3(a), $\alpha:=\underline{\lim } L\left(f_{i}\right)$ is a continuous $\mathbb{K}$-Lie algebra homomorphism. Abbreviate

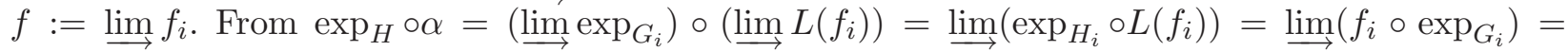

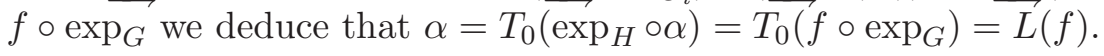

Now suppose that $h: G \rightarrow H$ is a continuous homomorphism. We may assume that $I=\mathbb{N}$ and $G_{1} \subseteq G_{2} \subseteq \cdots, H_{1} \subseteq H_{2} \subseteq \cdots$. After replacing $G$ by its identity component $G_{0}$, we may assume that each $G_{n}$ is connected. Using Proposition 3.4, we find $m(n) \in \mathbb{N}$ such that $h\left(G_{n}\right) \subseteq H_{m(n)}$, and such that $h_{n}:=\left.h\right|_{G_{n}} ^{H_{m(n)}}$ is continuous and hence $C_{\mathbb{R}}^{\omega}$. We may assume that $m(1)<m(2)<\cdots$; after passing to a cofinal subsequence of the $H_{n}$, without loss of generality $m(n)=n$ for each $n$. Thus, $h=\lim _{\longrightarrow} h_{n}$ is $c_{\mathbb{R}}^{\omega}$, by Theorem $4.3(\mathrm{f})$.

Remark 4.7. (a) The exponential map of a direct limit group need not be well behaved, as the example $G=\mathbb{C}^{\infty} \rtimes_{\alpha} \mathbb{R}=\lim _{\longrightarrow}\left(\mathbb{C}^{n} \rtimes \mathbb{R}\right)$ with $\alpha: \mathbb{R} \rightarrow \operatorname{Aut}\left(\mathbb{C}^{\infty}\right), \alpha(t)\left(\left(z_{k}\right)_{k \in \mathbb{N}}\right):=\left(e^{i k t} z_{k}\right)_{k \in \mathbb{N}}$ shows. Here $\exp _{G}$ fails to be injective on any zero-neighbourhood, and the exponential image $\operatorname{im}\left(\exp _{G}\right)$ is not an identity neighbourhood in $G$ [Glo03a, Example 5.5].

(b) As a consequence of (a), the exponential map $\exp _{H}$ of the complex analytic Lie group $H:=\mathbb{C}^{\infty} \rtimes_{\beta} \mathbb{C}=\underline{\lim }\left(\mathbb{C}^{n} \rtimes \mathbb{C}\right)$ with $\beta(z)\left(\left(z_{k}\right)_{k \in \mathbb{N}}\right):=\left(e^{i k z} z_{k}\right)_{k \in \mathbb{N}}$ is also not injective on any zero-neighbourhood. This settles an open problem by Milnor [Mil82, p. 31] in the negative, who asked whether every complex analytic Lie group is 'of Campbell-Hausdorff type'.

\section{Integration of locally finite Lie algebras}

A Lie algebra is locally finite if every finite subset generates a finite-dimensional subalgebra.

Theorem 5.1. Let $\mathfrak{g}$ be a countable-dimensional locally finite Lie algebra over $\mathbb{K} \in\{\mathbb{R}, \mathbb{C}\}$. Then there exists a $c_{\mathbb{K}}^{\omega}$-regular, $c_{\mathbb{K}}^{\omega}$-Lie group $G$, which also is a regular $C_{\mathbb{K}}^{\infty}$-Lie group in Milnor's sense, such that $L(G) \cong \mathfrak{g}$, equipped with the finite topology.

Proof. As $\mathfrak{g}$ is locally finite and $\operatorname{dim}_{\mathbb{K}}(\mathfrak{g}) \leqslant \aleph_{0}$, there is an ascending sequence $\mathfrak{g}_{1} \subseteq \mathfrak{g}_{2} \subseteq \cdots$ of finite-dimensional subalgebras of $\mathfrak{g}$, with union $\mathfrak{g}$. For each $n \in \mathbb{N}$, let $G_{n}$ be a simply connected $\mathbb{K}$-Lie group with Lie algebra $L\left(G_{n}\right) \cong \mathfrak{g}_{n}$; fix an isomorphism $\kappa_{n}: L\left(G_{n}\right) \rightarrow \mathfrak{g}_{n}$. If $m \geqslant n$, then the Lie algebra homomorphism $\kappa_{m, n}:=\kappa_{m}^{-1} \circ \kappa_{n}: L\left(G_{n}\right) \rightarrow L\left(G_{m}\right)$ (corresponding to the inclusion $\left.\operatorname{map} \mathfrak{g}_{n} \hookrightarrow \mathfrak{g}_{m}\right)$ induces a $C_{\mathbb{K}}^{\omega}$-homomorphism $\phi_{m, n}: G_{n} \rightarrow G_{m}$ such that $L\left(\phi_{m, n}\right)=\kappa_{m, n}$. Since $L\left(\phi_{k, m} \circ \phi_{m, n}\right)=L\left(\phi_{k, m}\right) \circ L\left(\phi_{m, n}\right)=\kappa_{k, m} \circ \kappa_{m, n}=\kappa_{k, n}=L\left(\phi_{k, n}\right)$, we have $\phi_{k, m} \circ \phi_{m, n}=\phi_{k, n}$ for all $k \geqslant m \geqslant n$, whence $\left(\left(G_{n}\right)_{n \in \mathbb{N}},\left(\phi_{m, n}\right)_{m \geqslant n}\right)$ is a direct system of $C_{\mathbb{K}}^{\omega}$-Lie groups. Now take $G:=\lim G_{n}$ in the category of $c_{\mathbb{K}}^{\omega}$-Lie groups. We shall presently show that, for each $n$, the normal subgroup $K_{n}:=\bigcup_{m \geqslant n} \operatorname{ker} \phi_{m, n}$ of $G_{n}$ is discrete. Hence, by Theorem $4.3, G$ is a $c_{\mathbb{K}}^{\omega}$-regular $c_{\mathbb{K}}^{\omega}$-Lie group, $G=\lim _{\longrightarrow} G_{n} / K_{n}$, and $L(G)=\lim _{\longrightarrow} L\left(G_{n} / K_{n}\right)=\lim _{\longrightarrow} L\left(G_{n}\right) \cong \lim _{\mathfrak{g}}=\mathfrak{g}$. For Milnor regularity, see Theorem 8.1.

Each $K_{n}$ is discrete. We show that the closure $N_{n}:=\overline{K_{n}} \subseteq G_{n}$ is discrete. The homomorphism $\phi_{m, n}$ has discrete kernel for all $m, n \in \mathbb{N}$ with $m \geqslant n$, because $L\left(\phi_{m, n}\right)=\kappa_{m, n}$ is injective. Now ker $\phi_{m, n}$ being a discrete normal subgroup of the connected group $G_{n}$, it is central. This entails that $N_{n} \subseteq Z\left(G_{n}\right)$, for each $n$, whence $\left(N_{n}\right)_{0} \subseteq Z\left(G_{n}\right)_{0}$ is a vector group being a connected closed subgroup of a vector group (Lemma 5.2). It is obvious from the definitions that $\phi_{m, n}\left(K_{n}\right) \subseteq K_{m}$ for all $m \geqslant n$, whence $\phi_{m, n}\left(N_{n}\right) \subseteq N_{m}$ and $\phi_{m, n}\left(\left(N_{n}\right)_{0}\right) \subseteq\left(N_{m}\right)_{0}$. Being a continuous homomorphism between vector groups, $\psi_{m, n}:=\left.\phi_{m, n}\right|_{\left(N_{n}\right)_{0}} ^{\left(N_{m}\right)_{0}}$ is real linear. Hence, being a real linear map with discrete kernel, $\psi_{m, n}$ is injective. Thus, $\left(N_{n}\right)_{0}=\overline{\bigcup_{m \geqslant n} \operatorname{ker} \psi_{m, n}}=\{1\}$, whence $N_{n}$ is discrete.

Here, we used the following fact. 


\section{FUndAMENTALS OF DIRECT LIMIT LIE THEORY}

Lemma 5.2. Let $G$ be a simply connected, finite-dimensional real Lie group. Then $Z(G)_{0}$ is a vector group, i.e. $Z(G)_{0} \cong \mathbb{R}^{m}$ for some $m \in \mathbb{N}_{0}$.

Proof. By Lévi's Theorem, $L(G)=\mathfrak{r} \rtimes \mathfrak{s}$ internally, where $\mathfrak{r}$ is the radical of $L(G)$ and $\mathfrak{s}$ a Lévi complement (see [Ser92, Part I, ch. VI, Theorem 4.1] or [Bou89, ch. I, $\S 6.8$, Theorem 5]). Let $R$ and $S$ be the analytic subgroups of $G$ corresponding to $\mathfrak{r}$ and $\mathfrak{s}$, respectively. Then $R$ and $S$ are simply connected, $R$ is a closed normal subgroup of $G, S$ a closed subgroup, and $G=R \rtimes S$ internally [HN91, Korollar III.3.16]. Now consider the identity component $Z(G)_{0}$ of the centre $Z(G)$ of $G$. Let $\pi: G \rightarrow S$ be the projection onto $S$, with kernel $R$. Then $\pi\left(Z(G)_{0}\right) \subseteq Z(S)_{0}=\{1\}$, entailing that $Z(G)_{0} \subseteq R$. Thus $Z(G)_{0}$ is an analytic subgroup of the simply connected solvable Lie group $R$, whence $Z(G)_{0}$ is simply connected [HN91, Satz III.3.31]. Being a simply connected abelian Lie group, $Z(G)_{0}$ is a vector group.

\section{Extension of sections in principal bundles}

We prove a preparatory result concerning sections in principal bundles, which will be used later to discuss closed subgroups and homogeneous spaces of direct limit groups.

Lemma 6.1. Given $\mathbb{K} \in\{\mathbb{R}, \mathbb{C}\}$, let $\pi_{1}: E_{1} \rightarrow M_{1}$ be a $C_{\mathbb{K}}^{\infty}$-map between finite-dimensional $C_{\mathbb{K}}^{\infty}$-manifolds and $\pi_{2}: E_{2} \rightarrow M_{2}$ be a finite-dimensional $G$-principal bundle of class $C_{\mathbb{K}}^{\infty}$ whose structure group $G$ is a finite-dimensional $C_{\mathbb{K}}^{\infty}$-Lie group. Let $m_{1}:=\operatorname{dim}_{\mathbb{K}}\left(M_{1}\right)$ and $m_{2}:=\operatorname{dim}_{\mathbb{K}}\left(M_{2}\right)$. Assume that $\lambda: M_{1} \rightarrow M_{2}$ is an injective $C_{\mathbb{K}}^{\infty}$-immersion and $\Lambda: E_{1} \rightarrow E_{2}$ a $C_{\mathbb{K}}^{\infty}$-map such that $\pi_{2} \circ \Lambda=\lambda \circ \pi_{1}$. Assume that $\phi_{1}: \Delta_{r}^{m_{1}}(\mathbb{K}) \rightarrow U_{1} \subseteq M_{1}$ is a chart for $M_{1}$, where $r>0$, and $\sigma_{1}: U_{1} \rightarrow E_{1}$ a $C_{\mathbb{K}}^{\infty}$-section of $\pi_{1}$. Then, for every $\left.s \in\right] 0, r\left[\right.$, there is a chart $\phi_{2}: \Delta_{s}^{m_{2}} \rightarrow U_{2}$ $\subseteq M_{2}$ and a $C_{\mathbb{K}}^{\infty}$-section $\sigma_{2}: U_{2} \rightarrow E_{2}$ of $\pi_{2}$ such that $\phi_{2}(x, 0)=\lambda\left(\phi_{1}(x)\right)$ for all $x \in \Delta_{s}^{m_{1}}$ and $\left.\sigma_{2} \circ \lambda\right|_{W}=\left.\Lambda \circ \sigma_{1}\right|_{W}$, where $W:=\phi_{1}\left(\Delta_{s}^{m_{1}}\right)$. If $\mathbb{K}=\mathbb{R}$ and all of $E_{1}, M_{1}, \pi_{1}$, the principal bundle $\pi_{2}, \lambda, \Lambda, \phi_{1}$ and $\sigma_{1}$ are real analytic, then also $\phi_{2}$ and $\sigma_{2}$ can be chosen as real analytic maps.

Proof. Since $\lambda \circ \phi_{1}$ is an injective immersion, there is a chart $\phi_{2}: \Delta_{s}^{m_{2}} \rightarrow U_{2} \subseteq M_{2}$ such that $\phi_{2}(x, 0)=\lambda\left(\phi_{1}(x)\right)$ for all $x \in \Delta_{s}^{m_{1}}$ (Lemma 2.3).

The $C_{\mathbb{K}}^{\infty}$-case. If $\mathbb{K}=\mathbb{R}$, then $\left.E_{2}\right|_{U_{2}}$ is trivial as a $G$-principal bundle of class $C_{\mathbb{R}}^{\infty}$, since $U_{2} \cong \Delta_{s}^{m_{2}}$ is paracompact and contractible (this is a well-known fact, which can be proved exactly as [Hir76, Corollary 4.2.5]). If $\mathbb{K}=\mathbb{C}$, then $\left.E_{2}\right|_{U_{2}}$ is trivial as a $G$-principal bundle of class $C_{\mathbb{C}}^{\infty}$, since $U_{2} \cong$ $\Delta_{s}^{m_{2}}(\mathbb{C})$ is a contractible Stein manifold [Gra58a, Satz 6].

Real analytic case. Since $U_{2} \cong \Delta_{s}^{m_{2}}$ is $\sigma$-compact and contractible, $\left.E_{2}\right|_{U_{2}}$ is trivial as a topological $G$-principal bundle and therefore also as a real analytic $G$-principal bundle, by injectivity of $\vartheta$ in [Tog67, Teorema 5].

By the preceding, in either case, we find a $C_{\mathbb{K}}^{\infty}$ (respectively $C_{\mathbb{R}}^{\omega}$ ) trivialization $\theta:\left.E_{2}\right|_{U_{2}} \rightarrow U_{2} \times G$. Let $\theta_{2}:\left.E_{2}\right|_{U_{2}} \rightarrow G$ be the second coordinate function of $\theta$. Define $\sigma_{2}:=\zeta \circ \phi_{2}^{-1}: U_{2} \rightarrow E_{2}$, where $\zeta: \Delta_{s}^{m_{2}} \rightarrow E_{2}$ is defined via

$$
\zeta(x, y):=\theta^{-1}\left(\phi_{2}(x, y), \theta_{2}\left(\left(\Lambda \circ \sigma_{1} \circ \phi_{1}\right)(x)\right)\right) \quad \text { for } x \in \Delta_{s}^{m_{1}}, y \in \Delta_{s}^{m_{2}-m_{1}} .
$$

Then $\sigma_{2}: U_{2} \rightarrow E_{2}$ is a $C_{\mathbb{K}}^{\infty}$-section (respectively $C_{\mathbb{R}}^{\omega}$-section) with the required properties.

\section{Fundamentals of Lie theory for direct limit groups}

In this section, we develop the basics of Lie theory for direct limit groups. Throughout the following, $G_{1} \subseteq G_{2} \subseteq \cdots$ is an ascending sequence of finite-dimensional Lie groups over $\mathbb{K} \in\{\mathbb{R}, \mathbb{C}\}$, such that the inclusion maps $\lambda_{n, m}: G_{m} \rightarrow G_{n}$ are $C_{\mathbb{K}^{-h}}^{\omega}$ omomorphisms, and $G:=\bigcup_{n \in \mathbb{N}} G_{n}$, equipped 


\section{H. GLÖCKNER}

with the $c_{\mathbb{K}^{\omega}}^{\omega}$-Lie group structure such that $G=\underline{\lim } G_{n}$ in the category of $c_{\mathbb{K}^{\omega}}^{\omega}$-Lie groups (and $C_{\mathbb{K}}^{\infty}$-Lie groups).

7.1 A map $f: M \rightarrow N$ between $c_{\mathbb{K}}^{\omega}$-manifolds is called $c_{\mathbb{K}}^{\omega}$-final if a map $g: N \rightarrow Z$ into a $c_{\mathbb{K}}^{\omega}$-manifold is $c_{\mathbb{K}}^{\omega}$ if and only if $g \circ f$ is $c_{\mathbb{K}}^{\omega}$. The map $f$ is $c_{\mathbb{K}}^{\omega}$-initial if a map $g: Z \rightarrow M$ from a $c_{\mathbb{K}}^{\omega}$-manifold $Z$ to $M$ is $c_{\mathbb{K}}^{\omega}$ if and only if $f \circ g$ is $c_{\mathbb{K}}^{\omega}$. Obvious adaptations are used to define $c_{\mathbb{K}}^{\infty}$-final, $C_{\mathbb{K}}^{r}$-final, $c_{\mathbb{K}}^{\infty}$-initial, and $C_{\mathbb{K}^{r}}^{r}$-initial maps, where $r \in \mathbb{N}_{0} \cup\{\infty\}$. A subset $M \subseteq N$ of a $c_{\mathbb{K}}^{\omega}$-manifold $M$ is called a (split) submanifold if there exists a (complemented) closed vector subspace $F$ of the modelling space $E$ of $N$ such that, for every $x \in M$, there exists a chart $\phi: U \rightarrow V \subseteq N$ of $N$ around $x$ such that $\phi(U \cap F)=M \cap V$. Then $M$, with the induced topology, can be made a $c_{\mathbb{K}}^{\omega}$-manifold modelled on $F$, in an apparent way.

Proposition 7.2 (Subgroups are Lie groups). Every subgroup $H \subseteq G$ admits a $c_{\mathbb{K}}^{\omega}$-Lie group structure with Lie algebra $L(H)=\left\{v \in L(G): \exp _{G}(\mathbb{K} v) \subseteq H\right\}=: \mathfrak{h}$ which makes the inclusion map $\lambda: H \rightarrow G$ a $C_{\mathbb{K}}^{\infty}$-homomorphism and both a $c_{\mathbb{K}}^{\omega}$-initial and a $c_{\mathbb{K}}^{\infty}$-initial map, and such that $L(\lambda): L(H) \rightarrow L(G)$ is an embedding of topological $\mathbb{K}$-vector spaces. Furthermore, $H=\lim _{n} H_{n}$ in the category of $c_{\mathbb{K}}^{\omega}$-Lie groups, where $H_{n}:=H \cap G_{n}$ is equipped with the finite-dimensional $\mathbb{K}$-Lie group structure induced by $G_{n}$.

Proof. We equip $H_{n}$ with the finite-dimensional $C_{\mathbb{K}}^{\omega}$-Lie group structure induced by $G_{n}$, which makes the inclusion map $\lambda_{n}: H_{n} \rightarrow G_{n}$ an immersion and a $C_{\mathbb{K}}^{\omega}$-initial and $C_{\mathbb{K}}^{\infty}$-initial map inside the category of finite-dimensional $C_{\mathbb{K}^{-}}^{\omega}$ and $C_{\mathbb{K}}^{\infty}$-manifolds, respectively (see [Bou89, ch. III, $\S 4.5$, Definition 3 and Proposition 9]. Then the inclusion maps $\mu_{n, m}: H_{m} \rightarrow H_{n}$ are $C_{\mathbb{K}}^{\omega}$-immersions for $n \geqslant m$; we give $H=\bigcup_{n \in \mathbb{N}} H_{n}$ the $c_{\mathbb{K}}^{\omega}$ Lie group structure such that $\left(H,\left(\mu_{n}\right)_{n \in \mathbb{N}}\right)=\underline{\lim }\left(\left(H_{n}\right)_{n \in \mathbb{N}},\left(\mu_{n, m}\right)\right)$ in the category of $c_{\mathbb{K}}^{\omega}$-Lie groups, where $\mu_{n}: H_{n} \rightarrow H$ is the inclusion map (see Theorem 4.3). Then $\lambda=\lim _{n} \lambda_{n}: H \rightarrow G$ is $c_{\mathbb{K}}^{\omega}$. We have $L(H)=\bigcup_{n \in \mathbb{N}} L\left(H_{n}\right)$ (with obvious identifications) and $L(G)=\bigcup_{n \in \mathbb{N}} L\left(G_{n}\right)$ by Theorem 4.3(a), and each of $L\left(\lambda_{n}\right): L\left(H_{n}\right) \rightarrow L\left(G_{n}\right)$ as well as $L(\lambda): L(H) \rightarrow L(G)$ is the respective inclusion map. Thus, $L(\lambda)$ is injective. Being an injective linear map between locally convex spaces equipped with their finest locally convex topologies, $L(\lambda)$ is a topological embedding (cf. [HM98, Proposition 7.25(ii)]). Clearly $L(H) \subseteq \mathfrak{h}$. If $v \in \mathfrak{h}$, then $v \in L\left(G_{n}\right)$ for some $n$ and thus $\exp _{G_{n}}(\mathbb{K} v) \subseteq G_{n} \cap H=H_{n}$, whence $v \in L\left(H_{n}\right) \subseteq L(H)$. Thus $L(H)=\mathfrak{h}$. Now assume that $M$ is a $c_{\mathbb{K}}^{\infty}$-manifold and $f: M \rightarrow H$ a map such that $\lambda \circ f: M \rightarrow G$ is $c_{\mathbb{K}}^{\infty}$. Then, for every smooth map $\gamma: \mathbb{K} \supseteq U \rightarrow M$ on an open zero-neighbourhood $U \subseteq \mathbb{K}$, the composition $f \circ \gamma$ maps some zero-neighbourhood $V \subseteq U$ into some $G_{n}$ and $\left.(f \circ \gamma)\right|_{V} ^{G_{n}}$ is $C_{\mathbb{K}}^{\infty}$, by Proposition 3.4. Since $f(\gamma(V)) \subseteq G_{n} \cap H=H_{n}$ and $H_{n}$ is $C_{\mathbb{K}}^{\infty}$-initial for maps from finitedimensional $C_{\mathbb{K}}^{\infty}$-manifolds, we deduce that $\left.(f \circ \gamma)\right|_{V} ^{H_{n}}$ is $C_{\mathbb{K}}^{\infty}$, whence $\left.(f \circ \gamma)\right|_{V}$ is $c_{\mathbb{K}}^{\infty}$. This entails that $f$ is $c_{\mathbb{K}}^{\infty}$. Thus, $\lambda: H \rightarrow G$ is $c_{\mathbb{K}}^{\infty}$-initial. Similarly, $\lambda$ is $c_{\mathbb{K}}^{\omega}$-initial.

Note that every $c_{\mathbb{C}}^{\omega}$-map between open subsets of $\mathbb{C}^{\infty}$ is $c_{\mathbb{R}}^{\omega}$. (In view of Lemma $1.9(\mathrm{~b})$ and the $c_{\mathbb{C}}^{\omega}$-analogue of Proposition 3.4 (Proposition 3.8), this follows from the fact that holomorphic maps between open subsets of finite-dimensional vector spaces are real analytic.) Hence, every direct limit $G=\underline{\lim } G_{n}$ of complex finite-dimensional Lie groups has an underlying $c_{\mathbb{R}}^{\omega}$ Lie group $G_{\mathbb{R}}$.

Lemma 7.3. If $\mathbb{K}=\mathbb{C}$ in the situation of Proposition 7.2, define $\mathfrak{h}$ as before and $\mathfrak{s}:=\{v \in L(G)$ : $\left.\exp _{G}(\mathbb{R} v) \subseteq H\right\}$. Let $S_{n}$ be $H_{n}$, equipped with the $C_{\mathbb{R}}^{\omega}$-Lie group structure induced by the $C_{\mathbb{R}}^{\omega}$-Lie group $\left(G_{n}\right)_{\mathbb{R}}$ underlying $G_{n}$, and define $S:=\lim S_{n}$. Thus $S=H$ as a set and an abstract group, and id $: H_{\mathbb{R}} \rightarrow S$ is $c_{\mathbb{R}}^{\omega}$. Then $\mathfrak{h}=\mathfrak{s}$ (as a set or real Lie algebra) if and only if $\left(H_{n}\right)_{\mathbb{R}}=S_{n}$ (as a real Lie group) for each $n \in \mathbb{N}$, if and only if $H_{\mathbb{R}}=S$ (as a $c_{\mathbb{R}}^{\omega}$-Lie group).

Proof. If $\mathfrak{h}=\mathfrak{s}$, then for every $n \in \mathbb{N}$ we have $L\left(S_{n}\right)+i L\left(S_{n}\right) \subseteq L\left(H_{m}\right)$ for some $m \geqslant n$. Let $v \in L\left(S_{n}\right)$. Then $\exp _{G_{n}}(\mathbb{C} v)=\exp _{G_{m}}(\mathbb{C} v) \subseteq H_{m} \cap G_{n}=H_{n}$, entailing that $v \in L\left(H_{n}\right)$. Thus $L\left(S_{n}\right) \subseteq L\left(H_{n}\right)$ and hence $L\left(S_{n}\right)=L\left(H_{n}\right)$, whence $S_{n}=\left(H_{n}\right)_{\mathbb{R}}$. 


\section{FUndAMENTALS OF DIRECT LIMIT LIE THEORY}

If $S_{n}=\left(H_{n}\right)_{\mathbb{R}}$ for each $n \in \mathbb{N}$, then $\left(\underline{\lim } H_{n}\right)_{\mathbb{R}}=\underline{\lim }\left(H_{n}\right)_{\mathbb{R}}=\underline{\lim } S_{n}$, by Theorem 4.3.

Now suppose that $H_{\mathbb{R}}=S$. We have $\mathfrak{h} \subseteq \mathfrak{s}$ by definition. If $v \in \mathfrak{s}$, then $\exp _{G}(\mathbb{R} v) \subseteq H$ and $\xi: \mathbb{R} \rightarrow S, \xi(t)=\exp _{G}(t v)=\exp _{S}(t v)$ is a $c_{\mathbb{R}}^{\omega}$-homomorphism. Since $S=H_{\mathbb{R}}=\underline{\lim }\left(H_{n}\right)_{\mathbb{R}}$ (see

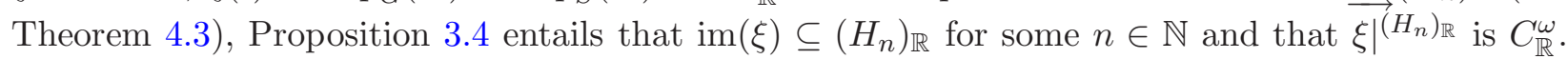
Hence, $\xi=\exp _{H_{n}}(\bullet w)$ for some $w \in L\left(H_{n}\right)$, where $w=v$ clearly and thus $\exp _{G}(\mathbb{C} v)=\exp _{H_{n}}(\mathbb{C} v) \subseteq$ $H_{n} \subseteq H$, whence $v \in \mathfrak{h}$. Therefore $\mathfrak{s}=\mathfrak{h}$.

7.4 We now specialize to the case where $H$ is a closed subgroup of $G$; if $\mathbb{K}=\mathbb{C}$, we assume that $\mathfrak{s}:=\left\{v \in L(G): \exp _{G}(\mathbb{R} v) \subseteq H\right\}$ is a complex Lie subalgebra of $L(G)$. Then the finitedimensional $C_{\mathbb{K}}^{\omega}$-Lie group structure induced by $G_{n}$ on its closed subgroup $H_{n}:=G_{n} \cap H$ makes $H_{n}$ a closed $C_{\mathbb{K}}^{\omega}$-submanifold of $G_{n}$ (this is obvious in the real case, and follows for $\mathbb{K}=\mathbb{C}$ using Lemma 7.3). For each $n \in \mathbb{N}$, we give $G_{n} / H_{n}$ the finite-dimensional $C_{\mathbb{K}}^{\omega}$-manifold structure making the canonical quotient map $q_{n}: G_{n} \rightarrow G_{n} / H_{n}$ a submersion. Let $q_{n, m}: G_{m} / H_{m} \rightarrow G_{n} / H_{n}$ be the uniquely determined $C_{\mathbb{K}}^{\omega}$-maps such that $q_{n, m} \circ q_{m}=q_{n} \circ \lambda_{n, m}$. Then $\mathcal{T}:=\left(\left(G_{n} / H_{n}\right)_{n \in \mathbb{N}},\left(q_{n, m}\right)_{n \geqslant m}\right)$ is a direct system of paracompact, finite-dimensional $C_{\mathbb{K}}^{\omega}$-manifolds and injective $C_{\mathbb{K}}^{\omega}$-immersions, whence $\left(M,\left(\psi_{n}\right)_{n \in \mathbb{N}}\right):=\lim \mathcal{T}$ exists in the category of $c_{\mathbb{K}}^{\omega}$-manifolds (Proposition 3.8). We have $\left(M,\left(\psi_{n}\right)_{n \in \mathbb{N}}\right)=\lim _{\longrightarrow} \mathcal{T}$ also in the categories of $C_{\mathbb{K}}^{\infty}$-manifolds, and the category of sets. Consider the quotient map $q: G \rightarrow G / H$ and the inclusion maps $\lambda_{n}: G_{n} \rightarrow G$. For each $n \in \mathbb{N}$, the map $q \circ \lambda_{n}$ factors to an injective map $\mu_{n}: G_{n} / H_{n} \rightarrow G / H$, determined by $\mu_{n} \circ q_{n}=q \circ \lambda_{n}$. Then $\left(G / H,\left(\mu_{n}\right)_{n \in \mathbb{N}}\right)$ is a cone over $\mathcal{T}$, and hence induces a map $\mu: M \rightarrow G / H$. It is easy to see that $\mu$ is a bijection; we give $G / H$ the $c_{\mathbb{K}}^{\omega}$-manifold structure making $\mu$ a $c_{\mathbb{K}}^{\omega}$-diffeomorphism; thus $G / H \cong \lim _{n} G_{n} / H_{n}$. Then also $\left(G / H,\left(\mu_{n}\right)_{n \in \mathbb{N}}\right)=\underline{\lim } \mathcal{T}$ in the category of $c_{\mathbb{K}}^{\omega}$-manifolds. Since $q=\lim _{\longrightarrow} q_{n}$, the map $q$ is $c_{\mathbb{K}}^{\omega}$.

Proposition 7.5 (Closed subgroups, quotient groups, homogeneous spaces). Let $H$ be a closed subgroup of $G$; if $\mathbb{K}=\mathbb{C}$, assume that $\left\{v \in L(G): \exp _{G}(\mathbb{R} v) \subseteq H\right\}$ is a complex Lie subalgebra of $G$. Equip $G / H$ with the $c_{\mathbb{K}}^{\omega}$-manifold structure described in $\S 7.4$; thus $G / H \cong \underline{\lim } G_{n} / H_{n}$ as a $c_{\mathbb{K}}^{\omega}$-manifold. Then the following hold.

(a) $q: G \rightarrow G / H$ admits local $c_{\mathbb{K}}^{\omega}$-sections, i.e. $q: G \rightarrow G / H$ is an $H$-principal bundle of class $c_{\mathbb{K}}^{\omega}$. Therefore, $q$ is $c_{\mathbb{K}}^{\omega}$-final, $c_{\mathbb{K}}^{\infty}$-final and $C_{\mathbb{K}}^{r}$-final, for each $r \in \mathbb{N}_{0} \cup\{\infty\}$.

(b) $H$ is a closed, split $c_{\mathbb{K}}^{\omega}$-submanifold of $G$. The $c_{\mathbb{K}}^{\omega}$-submanifold structure on $H$ and the $c_{\mathbb{K}}^{\omega}$-manifold structure underlying the $c_{\mathbb{K}}^{\omega}$-Lie group structure induced by $G$ on $H$ (as described in Proposition 7.2) coincide. This manifold structure makes the inclusion map $H \rightarrow G$ a $c_{\mathbb{K}}^{\omega}$-initial, $c_{\mathbb{K}}^{\infty}$-initial, and $C_{\mathbb{K}}^{r}$-initial map, for each $r \in \mathbb{N}_{0} \cup\{\infty\}$. If $L(H)=\{0\}$, then $H$ is discrete in the topology induced by $G$.

(c) If $H$ is furthermore a normal subgroup of $G$, then the $c_{\mathbb{K}}^{\omega}$-manifold structure on $G / H$ makes the quotient group $G / H$ a $c_{\mathbb{K}^{\omega}}^{\omega}$-regular $c_{\mathbb{K}^{\omega}}^{\omega}$ Lie group such that $G / H=\underline{\lim } G_{n} / H_{n}$ in the category of $c_{\mathbb{K}}^{\omega}$-Lie groups.

Proof. (a) Let $x \in G / H$; then there exists $k \in \mathbb{N}$ and $y \in G_{k}$ such that $x=q(y)$. Define $z:=q_{k}(y)$. Define $r_{n}:=1+2^{-n}$ for $n \in \mathbb{N}$, and $d_{n}:=\operatorname{dim}_{\mathbb{K}}\left(G_{n} / H_{n}\right)$. There exists a $C_{\mathbb{K}^{-}}^{\omega}$ section $\tau: V \rightarrow G_{k}$ of $q_{k}$ on some open neighbourhood $V$ of $z$ in $G_{k} / H_{k}$, and a chart $\phi_{k}: \Delta_{r_{k}}^{d_{k}} \rightarrow U_{k} \subseteq G_{k} / H_{k}$ such that $U_{k} \subseteq V$; we define $\sigma_{k}:=\left.\tau\right|_{U_{k}}$. Inductively, Lemma 6.1 provides charts $\phi_{n}: \Delta_{r_{n}}^{d_{n}} \rightarrow U_{n} \subseteq$ $G_{n} / H_{n}$ and $C_{\mathbb{K}^{-}}^{\omega}$ sections $\sigma_{n}: U_{n} \rightarrow G_{n}$ such that $\left.q_{n, m} \circ \phi_{m}\right|_{\Delta_{r_{n}}^{d_{m}}}=\left.\phi_{n}\right|_{\Delta_{r_{m}}^{d_{m}}}$ for all $n \geqslant m \geqslant k$ and $\sigma_{n}\left(q_{n, m}(w)\right)=\sigma_{m}(w)$ for all $w \in \phi_{m}\left(\Delta_{r_{n}}^{d_{m}}\right)$. Define $W_{n}:=\phi_{n}\left(\Delta_{1}^{d_{n}}\right)$ for $n \in \mathbb{N}, n \geqslant k$. Then $W:=\bigcup_{n \geqslant k} \mu_{n}\left(W_{n}\right)$ is an open subset of $G / H$, and $\left(W,\left(\left.\mu_{n}\right|_{W_{n}} ^{W}\right)_{n \geqslant k}\right)=\underline{\lim } \mathcal{W}$ as a $c_{\mathbb{K}}^{\omega}$-manifold, where $\mathcal{W}:=\left(\left(W_{n}\right)_{n \geqslant k},\left(\left.q_{n, m}\right|_{W_{m}} ^{W_{n}}\right)\right)$. Now $\sigma:=\lim _{\longrightarrow}\left(\left.\sigma\right|_{W_{n}}\right): W=\underline{\lim _{\longrightarrow}} W_{n} \rightarrow \underline{\lim } G_{n}=G$ is a $c_{\mathbb{K}}^{\omega}-\operatorname{map}$, 


\section{H. GLÖCKNER}

and it is a section for $q$ because $q \circ \sigma=\underline{\lim }\left(\left.q_{n} \circ \sigma_{n}\right|_{W_{n}}\right)=\lim _{\longrightarrow} j_{n}=j$, where $j_{n}: W_{n} \rightarrow G_{n} / H_{n}$ and $j: W \rightarrow G / H$ are the inclusion maps. The remainder is obvious.

(b) For the $c_{\mathbb{K}}^{\omega}$-Lie group structure induced by $G$ on $H$, we have $H=\lim _{\longrightarrow} H_{n}$ by Proposition 7.2, and this then also holds for the underlying $c_{\mathbb{K}}^{\omega}$ and $c_{\mathbb{K}}^{\infty}$-manifold structures (Theorem 4.3(d)-(f)). Hence, by the proof of Theorem 3.1, there exists a chart of $H$ around 1 of the form $\phi=\lim _{\longrightarrow} \phi_{n}$ : $P \rightarrow Q \subseteq H$, where, for each $n$, the map $\phi_{n}: P_{n} \rightarrow Q_{n} \subseteq H_{n}$ is a chart of $H_{n}$ around 1, defined on an open subset $P_{n} \subseteq \mathbb{K}^{h_{n}}$ (where $h_{n}:=\operatorname{dim}_{\mathbb{K}}\left(H_{n}\right)$ ), $P:=\bigcup_{n \in \mathbb{N}} P_{n} \subseteq \mathbb{K}^{t}$ (where $\left.t:=\sup \left\{h_{n}: n \in \mathbb{N}\right\} \in \mathbb{N}_{0} \cup\{\infty\}\right)$, and $Q:=\bigcup_{n \in \mathbb{N}} Q_{n} \subseteq H$. By the proof of (a), there exist charts $\psi_{n}: \Delta_{1}^{d_{n}} \rightarrow W_{n}$ onto open neighbourhoods $W_{n} \subseteq G_{n} / H_{n}$ of $q_{n}(1)$ (where $d_{n}:=\operatorname{dim}_{\mathbb{K}}\left(G_{n} / H_{n}\right)$ ) and $C_{\mathbb{K}^{-}}^{\omega}$ sections $\sigma_{n}: W_{n} \rightarrow G_{n}$ of $q_{n}$, such that $q_{n, m}\left(W_{m}\right) \subseteq W_{n}, q_{n, m} \circ \psi_{m}=\left.\psi_{n}\right|_{\Delta_{1}^{d_{m}}}$, and $\left.\sigma_{n} \circ q_{n, m}\right|_{W_{m}}=\sigma_{m}$ for all $m, n \in \mathbb{N}$ such that $n \geqslant m$. Define $V_{n}:=\operatorname{im}\left(\sigma_{n}\right) Q_{n} \subseteq G_{n}$ and

$$
\theta_{n}: \Delta_{1}^{d_{n}+h_{n}} \rightarrow V_{n}, \quad \theta_{n}(x, y):=\sigma_{n}\left(\psi_{n}(x)\right) \phi_{n}(y) \quad \text { for } x \in \Delta_{1}^{d_{n}}, y \in \Delta_{1}^{h_{n}} .
$$

Since $\sigma_{n}$ is a section of $q_{n}$, the map $\theta_{n}$ is easily seen to be injective. Using the inverse function theorem, one verifies that $V_{n}$ is open in $G_{n}$ and that $\theta_{n}$ is a $C_{\mathbb{K}^{-}}^{\omega}$ diffeomorphism onto $V_{n}$. Then $V:=\bigcup_{n \in \mathbb{N}} V_{n}$ is open in $G$, and $\theta:=\lim _{\longrightarrow} \theta_{n}: \lim _{\longrightarrow} \Delta_{1}^{d_{n}+h_{n}} \rightarrow V$ is a $c_{\mathbb{K}^{-}}^{\omega}$ diffeomorphism. Let $\zeta: \mathbb{K}^{s+t}=\lim \mathbb{K}^{d_{n}+h_{n}} \rightarrow \mathbb{K}^{s} \times \mathbb{K}^{t}$ be the natural isomorphism of topological vector spaces (cf. Proposition 3.7), and $\Omega:=\zeta\left(\bigcup_{n} \Delta_{1}^{d_{n}+h_{n}}\right) \subseteq \mathbb{K}^{s} \times \mathbb{K}^{t}$. Then $\kappa:=\left.\theta \circ \zeta^{-1}\right|_{\Omega}: \Omega \rightarrow V$ is a $C_{\mathbb{K}}^{\infty}$-diffeomorphism. By construction of $\theta$, we have $V \cap H=Q$ and $\kappa^{-1}(V \cap H)=\Omega \cap\left(\{0\} \times \mathbb{K}^{t}\right)$, where $\{0\} \times \mathbb{K}^{t}$ is a closed, complemented vector subspace of $\mathbb{K}^{s} \times \mathbb{K}^{t}$. Hence, $H$ is a split $c_{\mathbb{K}^{-}}^{\omega}$-submanifold of $G$. As the restriction of $\kappa$ to a submanifold chart of $H$ is the given chart $\phi$ of $H$, the submanifold structure and the above manifold structure on $H$ coincide. If $L(H)=0$, then the topology on the Lie group $H$ is discrete and hence so is the topology on $H$ as a submanifold of $G$, the induced topology.

(c) By construction, $\left(G / H,\left(\mu_{n}\right)\right)=\lim \left(\left(G_{n} / H_{n}\right),\left(q_{n, m}\right)\right)$ as a $c_{\mathbb{K}}^{\omega}$-manifold. Since each $q_{n, m}$ also is a homomorphism, Theorem 4.3 shows that there is a group structure on the $c_{\mathbb{K}}^{\omega}$-manifold $G / H$ making it a Lie group, and such that each $\mu_{n}$ becomes a homomorphism. This requirement entails that $q: G \rightarrow G / H$ is a homomorphism, whence the group structure on $G / H$ is the one of the quotient group. For the second assertion, see Theorem 4.3.

Proposition 7.5(a) entails that the surjection $q$ is an open map. Hence, $q$ is a topological quotient map, and the manifold $G / H$ carries the quotient topology.

Example 7.6. If $G_{n}$ closed in $G_{n+1}$ for each $n$ and $K_{n} \subseteq G_{n}$ a maximal compact subgroup such that $K_{1} \subseteq K_{2} \subseteq \cdots$, then $K:=\bigcup_{n \in \mathbb{N}} K_{n}$ is a closed subgroup of $G=\bigcup_{n \in \mathbb{N}} G_{n}$. In fact, $K_{m} \cap G_{n}=K_{n}$ for $m \geqslant n$ by maximality, whence $K \cap G_{n}=K_{n}$ is closed in $G_{n}$.

Proposition 7.7. If $f: G \rightarrow A$ is $C_{\mathbb{K}^{-}}^{\infty}$ (respectively $c_{\mathbb{K}^{-}}^{\infty}$ ) homomorphism from $G=\bigcup_{n \in \mathbb{N}} G_{n}$ into a $C_{\mathbb{K}}^{\infty}$-Lie group modelled on a locally convex space (respectively a $c_{\mathbb{K}}^{\infty}$-Lie group), then $H:=\operatorname{ker}(f)$ satisfies the hypotheses of Proposition 7.5, and $L(H)=\operatorname{ker} L(f)$.

Proof. In the complex case, $H \cap G_{n}=\operatorname{ker}\left(\left.f\right|_{G_{n}}\right)$ is a complex Lie subgroup of $G_{n}$ by Lemma 4.4, whence the specific hypothesis of Proposition 7.5 is satisfied, by Lemma 7.3. If $w \in \operatorname{ker} L(f)$, then $\xi: \mathbb{R} \rightarrow H, \xi(t):=f\left(\exp _{G}(t w)\right)$ is a $C_{\mathbb{R}^{-}}^{\infty}$ (respectively $c_{\mathbb{R}^{-}}^{\infty}$ ) homomorphism such that $\xi^{\prime}(0)=L(f)(w)=0$ and thus $\xi=1$ (see [Mil83, Lemma 7.1], [KM97, Lemma 36.7]). Hence, $\exp _{G}(\mathbb{K} w) \subseteq H$ and therefore $w \in L(H)=\left\{v \in L(G): \exp _{G}(\mathbb{K} v) \subseteq H\right\}$. The inclusion $L(H) \subseteq$ ker $L(f)$ is trivial.

Proposition 7.8 (Canonical factorization). Let $f: G \rightarrow A$ be a $c_{\mathbb{K}}^{\infty}$-homomorphism between direct limit groups, where $G$ is connected, $G=\bigcup_{n \in \mathbb{N}} G_{n}$, and $A=\bigcup_{n \in \mathbb{N}} A_{n}$. Equip $G / \operatorname{ker}(f)$ with 


\section{FUndAMENTALS OF DIRECT LIMIT LIE THEORY}

the $c_{\mathbb{K}}^{\omega}$-Lie group structure from Proposition 7.5(c), and $\mathrm{im}(f)$ with the $c_{\mathbb{K}}^{\omega}$-Lie group structure induced by $A$ (as in Proposition 7.2). Let $\bar{f}: G / \operatorname{ker}(f) \rightarrow \operatorname{im}(f)$ be the bijective homomorphism induced by $f$. Then $\bar{f}$ is a $c_{\mathbb{K}}^{\omega}$-diffeomorphism.

Proof. In view of Proposition 3.5, we may assume that each $G_{n}$ and $A_{n}$ is connected. Note that $\bar{f}$ is $c_{\mathbb{K}}^{\omega}$ because the inclusion map $\operatorname{im}(f) \rightarrow A$ is $c_{\mathbb{K}}^{\omega}$-initial and the quotient map $G \rightarrow G / \operatorname{ker}(f)$ is $c_{\mathbb{K}^{-}}^{\omega}$-final. Replacing $G$ with $G / \operatorname{ker}(f)$ and $A$ with $\operatorname{im}(f)$, we may therefore assume that $f$ is bijective, and have to show that $f^{-1}$ is $c_{\mathbb{K}}^{\omega}$. Then $L(f)$ is injective, by Proposition 7.7.

$L(f)$ is surjective. To see this, let $x \in L(A)=\bigcup_{n \in \mathbb{N}} L\left(A_{n}\right)$; define $\mathfrak{s}:=\mathbb{K} x$ and $S:=\exp _{A}(\mathfrak{s})$. If $x \notin \operatorname{im}(L(f))$, then $\mathfrak{h}_{n} \cap \mathfrak{s}=\{0\}$ for each $n \in \mathbb{N}$, where $\mathfrak{h}_{n}:=L(f)\left(L\left(G_{n}\right)\right)$. Given $n$, there exists $m \in \mathbb{N}$ such that $L\left(A_{m}\right) \supseteq \mathfrak{h}_{n} \cup \mathfrak{s}$. Let $H_{n}$ and $S_{n}$ be the analytic subgroups of $A_{m}$ with Lie algebras $\mathfrak{h}_{n}$ and $\mathfrak{s}$, respectively. Then $S=S_{n}$ as a set, and the group $H_{n} \cap S=H_{n} \cap S_{n}$ is countable, because $\mathfrak{h}_{n} \cap \mathfrak{s}=\{0\}$. Thus $S=\bigcup_{n \in \mathbb{N}}\left(S \cap H_{n}\right)$ is countable. However, $S$ is uncountable, a contradiction. Therefore, $x \in \operatorname{im}(L(f))$.

$f^{-1}$ is $c_{\mathbb{K}}^{\omega}$. As $A=\underline{\lim } A_{n}$, it suffices to show that $\left.f^{-1}\right|_{A_{n}}$ is $c_{\mathbb{K}}^{\omega}$, for each $n \in \mathbb{N}$. Fix $n$. There exists $m \in \mathbb{N}$ such that $L(f)\left(L\left(G_{m}\right)\right) \supseteq L\left(A_{n}\right)$. Let $B$ be the analytic subgroup of $G_{m}$ with Lie algebra $L(f)^{-1}\left(L\left(A_{n}\right)\right)$. Then $\left.f\right|_{B} ^{A_{n}}$ is a bijective $C_{\mathbb{K}^{\omega}}^{\omega}$ homomorphism between connected finite-dimensional $\mathbb{K}$-Lie groups and hence an isomorphism of $C_{\mathbb{K}^{-}}^{\omega}$ Lie groups. Thus, $\left.f^{-1}\right|_{A_{n}} ^{B}$ is $C_{\mathbb{K}}^{\omega}$, whence so is $\left.f^{-1}\right|_{A_{n}}$.

Proposition 7.9 (Universal covering group). If $G_{n}$ is connected for each $n \in \mathbb{N}$, let $\pi_{n}: \widetilde{G}_{n} \rightarrow G_{n}$ be the universal covering group, and $\widetilde{\lambda}_{n, m}: \widetilde{G}_{m} \rightarrow \widetilde{G}_{n}$ be the $C_{\mathbb{K}}^{\infty}$-homomorphism which lifts $\lambda_{n, m} \circ \pi_{m}$. Then $\left(\left(\widetilde{G}_{n}\right)_{n \in \mathbb{N}},\left(\widetilde{\lambda}_{n, m}\right)\right)$ is a direct system in the category of $C_{\mathbb{K}}^{\infty}$-Lie groups; let $\left(\widetilde{G},\left(\Lambda_{n}\right)_{n \in \mathbb{N}}\right)$ be its direct limit. Then $\widetilde{G}$ is simply connected, and the $C_{\mathbb{K}}^{\infty}$-homomorphism $\pi:=\underline{\lim } \pi_{n}: \widetilde{G} \rightarrow G$ is a universal covering map.

Proof. We cannot use Remark 3.9 because the $\widetilde{\lambda}_{n, m}$ need not be injective. We therefore proceed as follows. As any connected $C_{\mathbb{K}}^{\infty}$-Lie group, $G$ has a universal covering group $p: P \rightarrow G$; thus $G$ is a simply connected $C_{\mathbb{K}}^{\infty}$-Lie group and $p$ a $C_{\mathbb{K}}^{\infty}$-homomorphism with discrete kernel. Being a regular topological space and locally diffeomorphic to $L(G), P$ is smoothly Hausdorff and hence also is a $c_{\mathbb{K}}^{\infty}$-Lie group. By [KM97, Theorem 38.6], $P$ is a $c_{\mathbb{K}}^{\infty}$-regular Lie group. Let $\lambda_{n}: G_{n} \rightarrow G$ be the inclusion map. Since $P$ is $c_{\mathbb{K}}^{\infty}$-regular, $L\left(\lambda_{n}\right): L\left(\widetilde{G}_{n}\right)=L\left(G_{n}\right) \rightarrow L(G)=L(P)$ integrates to a $c_{\mathbb{K}}^{\infty}$-homomorphism $\alpha_{n}: \widetilde{G}_{n} \rightarrow P$ (Lemma 1.2, [KM97, Theorem 40.3]). Being a cone, $\left(P,\left(\alpha_{n}\right)\right)$ induces a $c_{\mathbb{K}}^{\infty}$-homomorphism $\alpha: \widetilde{G} \rightarrow P$, determined by $\alpha \circ \Lambda_{n}=\alpha_{n}$. Recall from Theorem 4.3 that $\widetilde{G}=\underline{\lim } \widetilde{G}_{n} / D_{n}$, where $D_{n}:=\operatorname{ker}\left(\Lambda_{n}\right)$ and where the limit map $\mu_{n}: \widetilde{G}_{n} / D_{n} \rightarrow \widetilde{G}$ is obtained by factoring $\Lambda_{n}$ over $\widetilde{G}_{n} \rightarrow \widetilde{G}_{n} / D_{n}$. Because $\pi \circ \Lambda_{n}=\lambda_{n} \circ \pi_{n}$, the subgroup $D_{n} \subseteq \operatorname{ker}\left(\pi_{n}\right)$ is discrete. Hence, $L(\widetilde{G})=\underline{\lim } L\left(\widetilde{G}_{n} / D_{n}\right)=\underline{\lim } L\left(\widetilde{G}_{n}\right)=\lim _{\longrightarrow} L\left(G_{n}\right)=L(G)$. It is easily verified that $L(\alpha)=\operatorname{id}_{L(G)}$ with respect to these identifications. Now $\widetilde{G}$ being $c_{\mathbb{K}}^{\infty}$-regular and $P$ simply connected, id : $L(P)=L(G) \rightarrow L(G)=L(\widetilde{G})$ induces a $c_{\mathbb{K}}^{\infty}$-homomorphism $\beta: P \rightarrow \widetilde{G}$, determined by $L(\beta)=\operatorname{id}_{L(G)}$. Since $L(\alpha \circ \beta)=\operatorname{id}_{L(G)}=L\left(\operatorname{id}_{P}\right)$, we have $\alpha \circ \beta=\operatorname{id}_{P}$ by [Mil83, Lemma 7.1]. Likewise, $\beta \circ \alpha=\mathrm{id}_{\widetilde{G}}$. Thus, $\widetilde{G} \cong P$ is the universal covering group.

Proposition 7.10 (Integration of Lie algebra homomorphisms). Assume that $G=\bigcup_{n \in \mathbb{N}} G_{n}$ is simply connected. Then the following hold.

(a) Every $\mathbb{K}$-Lie algebra homomorphism $\alpha: L(G) \rightarrow L(H)$ into the Lie algebra of a $c_{\mathbb{K}}^{\infty}$-regular $c_{\mathbb{K}}^{\infty}$-Lie group $H$ integrates to a $c_{\mathbb{K}}^{\infty}$-homomorphism $\beta: G \rightarrow H$ such that $L(\beta)=\alpha$. If $\mathbb{K}=\mathbb{R}$ and $H$ is a $c_{\mathbb{R}}^{\omega}$-regular $c_{\mathbb{R}}^{\omega}$-Lie group here, then $\beta$ is $c_{\mathbb{R}}^{\omega}$. 


\section{H. GLÖCKNER}

(b) Every $\mathbb{K}$-Lie algebra homomorphism $\alpha: L(G) \rightarrow L(H)$ into the Lie algebra of a $\mathbb{K}$-analytic $B C H$-Lie group (see [Glo02a]) integrates to a $C_{\mathbb{K}^{-}}^{\infty}$ (and $c_{\mathbb{K}^{-}}^{\omega}$ ) homomorphism $\beta: G \rightarrow H$.

Proof. (a) See [KM97, Theorem 40.3] and Lemma 1.2.

(b) Let $\left(\left(\widetilde{G}_{n}\right),\left(\widetilde{\lambda}_{n, m}\right)\right),\left(\widetilde{G},\left(\Lambda_{n}\right)\right), \pi_{n}: \widetilde{G}_{n} \rightarrow G_{n}$, and $\pi: \widetilde{G} \rightarrow G$ be as in Proposition 7.9 . Because $G$ is simply connected, the covering homomorphism $\pi$ is an isomorphism. Hence $\widetilde{G}=G$ and $\Lambda_{n}=\lambda_{n} \circ \pi_{n}$ without loss of generality, where $\lambda_{n}: G_{n} \rightarrow G$ is the inclusion map. Now $H$ and $\widetilde{G}_{n}$ being BCH, the homomorphism $\alpha_{n}:=\alpha \circ L\left(\Lambda_{n}\right)$ integrates to a $C_{\mathbb{K}^{-h o m o m o r p h i s m ~}}^{\omega}$ $\beta_{n}: \widetilde{G}_{n} \rightarrow H$ (see [Glo02a, Proposition 2.8]). Then $\left(H,\left(\beta_{n}\right)\right)$ is a cone and hence induces a $C_{\mathbb{K}^{-}}^{\infty}\left(\right.$ and $\left.c_{\mathbb{K}^{-}}^{\omega}\right)$ homomorphism $\beta: G \rightarrow H$ such that $\beta \circ \Lambda_{n}=\beta_{n}$. Clearly $L(\beta)=\alpha$.

Proposition 7.11 (Integration of Lie subalgebras). Given a $\mathbb{K}$-Lie subalgebra $\mathfrak{h}$ of $L(G)$, equip the subgroup $H:=\left\langle\exp _{G}(\mathfrak{h})\right\rangle$ with the $c_{\mathbb{K}}^{\omega}$-Lie group structure described in Proposition 7.2. Then $H$ is connected, and $L(H)=\mathfrak{h}$. Furthermore, $H=\lim _{\longrightarrow} H_{n}$ where $H_{n}:=\left\langle\exp _{G_{n}}\left(\mathfrak{h}_{n}\right)\right\rangle$ is the analytic subgroup of $G_{n}$ with Lie algebra $\mathfrak{h}_{n}:=\mathfrak{h} \cap L\left(G_{n}\right)$.

Proof. Consider the inclusion map $f: S \rightarrow G$, where $S:=\lim _{\longrightarrow} H_{n}=\bigcup_{n \in \mathbb{N}} H_{n}$. Then $S=H$ as an abstract group. We have $L(S)=\bigcup_{n \in \mathbb{N}} L\left(H_{n}\right)=\mathfrak{h}$, and $\vec{f}$ is $c_{\mathbb{K}}^{\omega}$ because each $\left.f\right|_{H_{n}}$ is so. By Proposition $7.8, f$ is a $c_{\mathbb{K}}^{\omega}$-diffeomorphism onto $\operatorname{im}(f)=H$, equipped with the $c_{\mathbb{K}}^{\omega}$-Lie group structure induced by $G$. Thus, $S=H$ as $c_{\mathbb{K}^{\omega}}^{\omega}$ Lie groups.

Before we can discuss universal complexifications of direct limit groups, we need to re-examine universal complexifications of finite-dimensional Lie groups.

Lemma 7.12. Let $G$ be a finite-dimensional real Lie group, and $\gamma_{G}: G \rightarrow G_{\mathbb{C}}$ be its universal complexification in the category of finite-dimensional complex Lie groups. Let $\alpha: G \rightarrow H$ be a $c_{\mathbb{R}}^{\infty}$-homomorphism from $G$ to a $c_{\mathbb{C}}^{\omega}$-regular $c_{\mathbb{C}^{-}}^{\omega}$ Lie group $H$. Then there exists a unique $c_{\mathbb{C}}^{\omega}$-homomorphism $\beta: G_{\mathbb{C}} \rightarrow H$ such that $\beta \circ \gamma_{G}=\alpha$.

Proof. We assume first that $G$ is connected. Let $p: \widetilde{G} \rightarrow G$ be the universal covering group of $G$ and $S$ be a simply connected complex Lie group with Lie algebra $L(G)_{\mathbb{C}}$. Let $\lambda: L(G) \rightarrow L(G)_{\mathbb{C}}$ be the inclusion map and $\kappa: \widetilde{G} \rightarrow S$ be the unique $c_{\mathbb{R}}^{\infty}$-homomorphism such that $L(\kappa)=\lambda$. Set $\Pi:=\operatorname{ker}(p) \cong \pi_{1}(G)$ and let $N \subseteq S$ be the smallest closed complex Lie subgroup such that $\kappa(\Pi) \subseteq N$. Let $q: S \rightarrow S / N=: G_{\mathbb{C}}$ be the canonical quotient map. Then there exists a $c_{\mathbb{R}^{-}}^{\infty}$ homomorphism $\gamma_{G}: G \rightarrow G_{\mathbb{C}}$ such that $\gamma_{G} \circ p=q \circ \kappa$.

Let $\alpha: G \rightarrow H$ be a $c_{\mathbb{R}}^{\infty}$-homomorphism into a $c_{\mathbb{C}}^{\omega}$-regular $c_{\mathbb{C}}^{\omega}$-Lie group $H$. Lemma 1.2 provides a $c_{\mathbb{C}}^{\omega}$-homomorphism $\eta: S \rightarrow H$ such that $L(\eta)$ is the $\mathbb{C}$-linear extension of $L(\alpha)$. Then $\eta \circ \kappa=\alpha \circ p$, because $L(\eta \circ \kappa)=L(\alpha)=L(\alpha \circ p)$. Thus $\kappa(\Pi) \subseteq \operatorname{ker}(\eta)$, where $\operatorname{ker}(\eta)$ is a closed, complex Lie subgroup of $S$ by Lemma 4.4. Thus $N \subseteq \operatorname{ker}(\eta)$, and thus $\eta$ factors to a $c_{\mathbb{C}}^{\omega}$-homomorphism $\beta: G_{\mathbb{C}}=S / N \rightarrow H$ such that $\beta \circ q=\eta$. From $\beta \circ \gamma_{G} \circ p=\beta \circ q \circ \kappa=\eta \circ \kappa=\alpha \circ p$ we deduce that $\beta \circ \gamma_{G}=\alpha$, and clearly $\beta$ is uniquely determined by this property. By the preceding, $\gamma_{G}: G \rightarrow G_{\mathbb{C}}$ is a universal complexification of the $c_{\mathbb{R}}^{\infty}$-Lie group $G$ in the category of $c_{\mathbb{C}}^{\omega}$-regular $c_{\mathbb{C}}^{\omega}$-Lie groups; since $G_{\mathbb{C}}$ is finite-dimensional, $\gamma_{G}: G \rightarrow G_{\mathbb{C}}$ also is the universal complexification of $G$ in the category of finite-dimensional complex Lie groups.

If $G$ is not necessarily connected, then its identity component $G_{0}$ has a universal complexification in the category $\mathcal{A}$ of $c_{\mathbb{C}}^{\omega}$-regular $c_{\mathbb{C}}^{\omega}$-Lie groups, which is finite-dimensional. As in [Glo02a, Proposition 5.2], we see that the $c_{\mathbb{R}}^{\infty}$-Lie group $G$ has a universal complexification $\gamma_{G}: G \rightarrow G_{\mathbb{C}}$ in $\mathcal{A}$, and $\left(G_{\mathbb{C}}\right)_{0}$ is a universal complexification for $G_{0}$ and therefore finite-dimensional. Hence, $G_{\mathbb{C}}$ is finite-dimensional, and hence it coincides with the universal complexification of $G$ in the category of finite-dimensional complex Lie groups. 


\section{FUndAMENTALS OF DIRECT LIMIT LIE THEORY}

Proposition 7.13 (Universal complexifications). Let $\mathcal{S}:=\left(\left(G_{n}\right)_{n \in \mathbb{N}},\left(\lambda_{n, m}\right)_{n \geqslant m}\right)$ be a direct system of finite-dimensional real Lie groups and $C_{\mathbb{R}}^{\omega}$-homomorphisms, $\left(G,\left(\lambda_{n}\right)\right):=\lim \mathcal{S}$ in the category of $c_{\mathbb{R}}^{\omega}$-Lie groups and $\left(G_{\mathbb{C}},\left(\kappa_{n}\right)_{n \in \mathbb{N}}\right):=\underline{\lim }\left(\left(\left(G_{n}\right)_{\mathbb{C}}\right)_{n \in \mathbb{N}},\left(\left(\lambda_{n, m}\right)_{\mathbb{C}}\right)\right)$ in the category of $c_{\mathbb{C}}^{\omega}$-Lie groups, where $\gamma_{n}: G_{n} \rightarrow\left(G_{n}\right)_{\mathbb{C}}$ is a universal complexification for $G_{n}$ in the category of finite-dimensional complex Lie groups, and $\left(\lambda_{n, m}\right)_{\mathbb{C}}:\left(G_{m}\right)_{\mathbb{C}} \rightarrow\left(G_{n}\right)_{\mathbb{C}}$ the uniquely determined complex analytic homomorphism such that $\left(\lambda_{n, m}\right)_{\mathbb{C}} \circ \gamma_{m}=\gamma_{n} \circ \lambda_{n, m}$. Let $\gamma_{G}:=\lim _{\longrightarrow} \gamma_{n}: G \rightarrow G_{\mathbb{C}}$. Then the following hold:

(a) $\gamma_{G}: G \rightarrow G_{\mathbb{C}}$ is a universal complexification of the $c_{\mathbb{R}}^{\infty}$-Lie group $G$ in the category of $c_{\mathbb{C}}^{\omega}$-regular $c_{\mathbb{C}}^{\omega}$-Lie groups in the sense that for every $c_{\mathbb{R}}^{\infty}$-homomorphism $\alpha: G \rightarrow H$ into a $c_{\mathbb{C}}^{\omega}$-regular $c_{\mathbb{C}}^{\omega}$-Lie group $H$, there is a unique $c_{\mathbb{C}}^{\omega}$-homomorphism $\beta: G_{\mathbb{C}} \rightarrow H$ such that $\beta \circ \gamma_{G}=\alpha$.

(b) $\left.\gamma_{G}\right|_{G_{0}} ^{\left(G_{\mathbb{C}}\right)_{0}}$ is a universal complexification of $G_{0}$, and $G / G_{0} \rightarrow G_{\mathbb{C}} /\left(G_{\mathbb{C}}\right)_{0}, x G_{0} \mapsto \gamma_{G}(x)\left(G_{\mathbb{C}}\right)_{0}$ is a bijection.

(c) If $G$ is simply connected, then $G_{\mathbb{C}}$ is simply connected, and $\gamma_{G}$ has discrete kernel.

(d) If $\gamma_{G}$ has discrete kernel, then $L\left(G_{\mathbb{C}}\right)=L(G)_{\mathbb{C}}$, im $\gamma_{G}$ is closed in $G_{\mathbb{C}}$, and $\left.\gamma_{G}\right|^{\operatorname{im} \gamma_{G}}$ is a local $c_{\mathbb{R}}^{\omega}$-diffeomorphism onto im $\gamma_{G}$, equipped with the $c_{\mathbb{R}}^{\omega}$-Lie group structure induced by $\left(G_{\mathbb{C}}\right)_{\mathbb{R}}$.

Proof. (a) By Lemma 7.12, for each $n \in \mathbb{N}$ there exists a unique $c_{\mathbb{C}^{-}}^{\omega}$ homomorphism $\beta_{n}:\left(G_{n}\right)_{\mathbb{C}} \rightarrow H$ such that $\beta_{n} \circ \gamma_{n}=\alpha \circ \lambda_{n}$. Clearly $\left(H,\left(\beta_{n}\right)\right)$ is a cone, whence there exists a unique $c_{\mathbb{C}}^{\omega}$-homomorphism $\beta: G_{\mathbb{C}}=\lim _{\longrightarrow}\left(G_{n}\right)_{\mathbb{C}} \rightarrow H$ such that $\beta \circ \kappa_{n}=\beta_{n}$. Then $\beta \circ \gamma_{G}=\alpha$, and it is easily verified that $\beta$ is uniquely determined by this property.

(b) Compare [Glo02a, Proposition 5.2].

(c) Using Theorem 5.1, we find a simply connected, $c_{\mathbb{C}}^{\omega}$-regular $c_{\mathbb{C}}^{\omega}$-Lie group $S$ with Lie algebra $L(S)=L(G)_{\mathbb{C}}$. As $G$ is simply connected, the inclusion map $j: L(G) \hookrightarrow L(G)_{\mathbb{C}}$ integrates to a $c_{\mathbb{R}}^{\infty}$-homomorphism $\eta: G \rightarrow S$ such that $L(\eta)=j$. Then $\eta: G \rightarrow S$ is a universal complexification for $G$ in the category of $c_{\mathbb{C}}^{\omega}$-regular $c_{\mathbb{C}}^{\omega}$-Lie groups (cf. proof of Lemma 7.12 or [GN03, Lemma IV.4]). Let $K:=\operatorname{ker}(\eta)=\operatorname{ker}\left(\gamma_{G}\right)$. Because $L(\eta)=j$ is injective, we have $L(K)=\operatorname{ker} L(\eta)=\{0\}$ (Proposition 7.7). Hence, $K$ is discrete when equipped with the real Lie group structure induced by $G$. The topology on the latter coincides with the topology induced by $G$, as $K$ is closed (Proposition 7.5(b)). Hence $\operatorname{ker}\left(\gamma_{G}\right)=K$ is discrete.

(d) Since $\operatorname{ker}\left(\gamma_{G}\right)$ is discrete, $L\left(\gamma_{G}\right)$ is injective (Proposition 7.7), enabling us to identify $L(G)$ with $\operatorname{im} L\left(\gamma_{G}\right)$ as a real locally convex space. Let $\left(G_{\mathbb{C}}\right)_{\text {op }}$ be $G_{\mathbb{C}}$, equipped with the opposite complex structure; by the universal property of $G_{\mathbb{C}}$, there is a unique $c_{\mathbb{C}}^{\omega}$-homomorphism $\sigma: G_{\mathbb{C}} \rightarrow\left(G_{\mathbb{C}}\right)_{\text {op }}$ such that $\sigma \circ \gamma_{G}=\gamma_{G}$. We now consider $\sigma$ as an antiholomorphic self-map of $G_{\mathbb{C}}$. Thus $L(\sigma)$ is $\mathbb{C}$-antilinear. As in [GN03, Lemma IV.2], we see that $\sigma$ is an involution. We have $L(G) \subseteq L\left(G_{\mathbb{C}}\right)^{\sigma}$ for the fixed space of $L(\sigma)$. Since $L\left(G_{\mathbb{C}}\right)=L(G)+i L(G)$ by construction of $G_{\mathbb{C}}$, it easily follows that $L\left(G_{\mathbb{C}}\right)=L(G) \oplus i L(G)=L(G)_{\mathbb{C}}$ and thus $L(G)=L\left(G_{\mathbb{C}}\right)^{\sigma}$. We now give the closed subgroup $\left(G_{\mathbb{C}}\right)^{\sigma}:=\operatorname{Fix}(\sigma)$ the $c_{\mathbb{R}}^{\omega}$-Lie group structure induced by $\left(G_{\mathbb{C}}\right)_{\mathbb{R}}$. Then $\gamma_{G}(G) \subseteq\left(G_{\mathbb{C}}\right)^{\sigma}$, and it is easy to see that $L\left(\left(G_{\mathbb{C}}\right)^{\sigma}\right):=\left\{v \in L\left(G_{\mathbb{C}}\right): \exp _{G_{\mathbb{C}}}(\mathbb{R} v) \subseteq\left(G_{\mathbb{C}}\right)^{\sigma}\right\}=L(G)$. Thus $C:=\left(\left(G_{\mathbb{C}}\right)^{\sigma}\right)_{0}=$ $\left\langle\exp _{G_{\mathbb{C}}}(L(G))\right\rangle=\gamma_{G}\left(G_{0}\right)$, and now Proposition 7.8 entails that $\left.\gamma_{G}\right|_{G_{0}} ^{C}$ is a local $c_{\mathbb{R}^{-}}^{\omega}$-diffeomorphism. To complete the proof, note that $\left(G_{\mathbb{C}}\right)_{0} \cap \gamma_{G}(G)=\gamma_{G}\left(G_{0}\right)=C$ by (b), whence $\gamma_{G}(G)$ is a locally closed subgroup of $G_{\mathbb{C}}$ and hence closed.

\section{Proof of regularity in Milnor's sense}

Theorem 8.1. Every direct limit group $G=\underline{\lim } G_{n}$ over $\mathbb{K} \in\{\mathbb{R}, \mathbb{C}\}$ is a regular $C_{\mathbb{R}}^{\infty}$-Lie group in Milnor's sense. More precisely, for every $k \in \mathbb{N} \cup\{\infty\}$, every $C_{\mathbb{R}}^{k}$-curve $\gamma:[0,1] \rightarrow G$ admits a right product integral $\eta=\operatorname{Evol}_{G}^{r}(\gamma) \in C^{k+1}([0,1], G)$ such that $\eta(0)=1$, and the corresponding 


\section{H. GLÖCKNER}

right evolution map

$$
\operatorname{evol}_{G}^{r}: C^{k}([0,1], L(G)) \rightarrow G, \operatorname{evol}_{G}^{r}(\gamma):=\operatorname{Evol}_{G}^{r}(\gamma)(1)
$$

is $C_{\mathbb{K}}^{\infty}$ and $c_{\mathbb{K}}^{\omega}$.

Proof. Fix $k$. The strategy of the proof is as follows. First, we show that product integrals exist and that $\operatorname{evol}_{G}^{r}$ is continuous. Next, we show that $\operatorname{evol}_{G}^{r}$ is complex analytic if $\mathbb{K}=\mathbb{C}$. Finally, for $\mathbb{K}=\mathbb{R}$, we deduce smoothness of $\operatorname{evol}_{G}^{r}$ from the smoothness of $\operatorname{evol}_{G_{\mathbb{C}}}^{r}$.

Step 1. Since $\operatorname{evol}_{G}^{r}$ takes its values in the connected component of $G$, we may assume that $G$ is connected. Using that $\delta^{r}(p \circ \gamma)=\delta^{r} \gamma$ for curves in $\widetilde{G}$ (cf. [KM97, 38.4(3)]), where $p: \widetilde{G} \rightarrow G$ is the universal covering map, we may assume that $G$ is simply connected. Furthermore, we may assume that $G=\bigcup_{n \in \mathbb{N}} G_{n}$, where $G_{1} \subseteq G_{2} \subseteq \cdots$ and each $G_{n}$ is connected. Let $j_{n}: G_{n} \rightarrow G$ be the inclusion map. We abbreviate $d_{n}:=\operatorname{dim}_{\mathbb{K}}\left(G_{n}\right), s:=\sup \left\{d_{n}: n \in \mathbb{N}\right\}$ and let $\phi=\lim _{\longrightarrow} \phi_{n}: P \rightarrow Q$ be a chart of $G$ around 1 , where $P=\bigcup_{n \in \mathbb{N}} \Delta_{2}^{d_{n}}, Q:=\bigcup_{n \in \mathbb{N}} Q_{n}$ and $\phi_{n}: \Delta_{2}^{d_{n}} \rightarrow Q_{n}$ is a chart of $G_{n}$ around 1 , such that $\phi_{n}(0)=1$. We identify $L\left(G_{n}\right)=T_{1}\left(G_{n}\right)$ with $\mathbb{K}^{d_{n}}$ using the chart $\phi_{n}$, and $L(G)$ with $\mathbb{K}^{s}$ using $\phi$; then $L\left(j_{n}\right): \mathbb{K}^{d_{n}} \rightarrow \mathbb{K}^{s}$ is the inclusion map, for each $n \in \mathbb{N}$.

Step 2: $\operatorname{evol}_{G}^{r}$ exists. To see this, let $\gamma \in C^{k}([0,1], L(G))$. Then there exists $n \in \mathbb{N}$ such that $\operatorname{im} \gamma \subseteq L\left(G_{n}\right)$. Then $\left.\gamma\right|^{L\left(G_{n}\right)}$ is $C^{k}$. It is a standard fact (based on the local existence and uniqueness of solutions to differential equations) that there exists $\eta \in C^{k+1}\left([0,1], G_{n}\right)$ such that $\delta^{r} \eta=\left.\gamma\right|^{L\left(G_{n}\right)}$. Then $\operatorname{Evol}_{G}^{r}(\gamma):=j_{n} \circ \eta$ is $C^{k+1}$ and $\delta^{r}\left(j_{n} \circ \eta\right)=\left.L\left(j_{n}\right) \circ \gamma\right|^{L\left(G_{n}\right)}=\gamma$. Thus $\operatorname{evol}_{G}^{r}(\gamma)$ exists, and $\operatorname{evol}_{G}^{r} \circ C^{k}\left([0,1], L\left(j_{n}\right)\right)=j_{n} \circ \operatorname{evol}_{G_{n}}^{r}$.

Step 3. The inclusion map $C^{k}([0,1], L(G)) \rightarrow C^{1}([0,1], L(G))$ being continuous linear for each $k$, it suffices to prove that $\operatorname{evol}_{G}^{r}: C^{1}([0,1], L(G)) \rightarrow G$ is $C_{\mathbb{K}}^{\infty}$ and $c_{\mathbb{K}}^{\omega}$. We may therefore assume that $k=1$ for the rest of the proof.

Step 4: $\operatorname{evol}_{G}^{r}$ is continuous at nice $\gamma_{0}$. We show that $\operatorname{evol}_{G}^{r}$ is continuous at $\gamma_{0} \in C^{1}([0,1], L(G))$, provided that $\operatorname{im}\left(\gamma_{0}\right) \subseteq \mathbb{K}^{d_{1}}=L\left(G_{1}\right)$ and $\operatorname{im}\left(\eta_{0}\right) \subseteq \phi_{1}\left(\Delta_{1 / 2}^{d_{1}}\right)$, where $\eta_{0}:=\operatorname{Evol}_{G}^{r}\left(\gamma_{0}\right)$. To this end, let $W$ be an open neighbourhood of $\operatorname{evol}_{G}^{r}\left(\gamma_{0}\right)=\eta_{0}(1)$ in $G$; abbreviate $\zeta_{0}:=\phi_{1}^{-1} \circ \eta_{0}$. Then $\phi^{-1}(W)$ is an open neighbourhood of $\zeta_{0}(1)$, whence $\phi^{-1}(W)-\zeta_{0}(1) \supseteq \Delta_{\varepsilon_{1}}^{d_{1}} \oplus \bigoplus_{n \geqslant 2} \Delta_{\varepsilon_{n}}^{d_{n}-d_{n-1}}$ for certain $\varepsilon_{n}>0$; we may assume that $1 \geqslant \varepsilon_{1} \geqslant \varepsilon_{2} \geqslant \cdots$. Define $r_{n}:=1-2^{-n}$ for $n \in \mathbb{N}$. Equip each $\mathbb{K}^{d_{n}}$ with the supremum norm. There is $R>0$ such that $\left\|\gamma_{0}\right\|_{\infty} \leqslant R$.

For $n \in \mathbb{N}$, consider the map $f_{n}: \mathbb{K}^{d_{n}} \times \Delta_{2}^{d_{n}} \rightarrow \mathbb{K}^{d_{n}}, f_{n}(y, x):=d /\left.d s\right|_{s=0} \phi_{n}^{-1}\left(\phi_{n}(s y) \phi_{n}(x)\right)$, which expresses the map $L\left(G_{n}\right) \times G_{n} \rightarrow T G_{n},(y, x) \mapsto T_{1}\left(\rho_{x}\right) \cdot y$ (with right translation $\rho_{x}: G_{n} \rightarrow G_{n}$ ) in local coordinates (forgetting the fibre). Then $\zeta_{0}^{\prime}(t)=f_{n}\left(\gamma_{0}(t), \zeta_{0}(t)\right)$ for all $t \in[0,1]$, because $\delta^{r}\left(\eta_{0}\right)=\gamma_{0}$. By the compactness of $\bar{\Delta}_{1}^{d_{n}}$ and $\bar{\Delta}_{R+n-1}^{d_{n}}$, there exists $k_{n}>0$ such that for the operator norms of the partial differentials we have

$$
\left\|d_{2} f_{n}(v, x, \bullet)\right\| \leqslant k_{n} \quad \text { for all } v \in \Delta_{R+n-1}^{d_{n}} \text { and } x \in \Delta_{1}^{d_{n}},
$$

and such that for the operator norms of the continuous linear maps $f_{n}(\bullet, x)$ we have $\left\|f_{n}(\bullet, x)\right\| \leqslant k_{n}$ for all $x \in \Delta_{1}^{d_{n}}$. Choose $\alpha_{n}>0$ so small that

$$
\frac{\alpha_{n}}{k_{n}}\left(e^{k_{n}}-1\right) \leqslant 2^{-n-1} \varepsilon_{n} .
$$

Define $s_{n}:=\min \left\{\alpha_{n} / k_{n}, 1\right\}$. Suppose that $\gamma:[0,1] \rightarrow \Delta_{R+n-1}^{d_{n}}$ is a $C^{1}$-curve for which there exists a $C^{1}$-curve $\eta:[0,1] \rightarrow \Delta_{1-2^{-n}}^{d_{n}}$ solving the initial value problem $\eta(0)=0, \eta^{\prime}(t)=f_{n}(\gamma(t), \eta(t))$. Then $\left\|d_{2} f_{n}(\gamma(t), x, \bullet)\right\| \leqslant k_{n}$ for all $t \in[0,1]$ and $x \in \Delta_{1}^{d_{n}}$. Let $\bar{\gamma}:[0,1] \rightarrow \mathbb{K}^{d_{n}}$ be a $C^{1}$-curve such that $\|\bar{\gamma}-\gamma\|_{\infty}<s_{n}$. Then $\operatorname{im}(\bar{\gamma}) \subseteq \Delta_{R+n}^{d_{n}}$, and

$$
\left\|f_{n}(\bar{\gamma}(t), x)-f_{n}(\gamma(t), x)\right\|=\left\|f_{n}(\bar{\gamma}(t)-\gamma(t), x)\right\| \leqslant\left\|f_{n}(\bullet, x)\right\| \cdot\|\bar{\gamma}(t)-\gamma(t)\| \leqslant k_{n} s_{n} \leqslant \alpha_{n}
$$




\section{FUndAMENTALS OF DIRECT LIMIT LIE THEORY}

for all $x \in \Delta_{1}^{d_{n}}$. Furthermore, $\eta(t)+y \in \Delta_{1-2^{-n-1}}^{d_{n}} \subseteq \Delta_{1}^{d_{n}}$ for all $t \in[0,1]$ and $y \in \mathbb{K}^{d_{n}}$ such that $\|y\| \leqslant\left(\alpha_{n} / k_{n}\right)\left(e^{k_{n}}-1\right) \leqslant 2^{-n-1} \varepsilon_{n} \leqslant 2^{-n-1}$. Using [Die60, (10.5.6)], we therefore find a solution $\xi:[0,1] \rightarrow \Delta_{1}^{d_{n}}$ to the initial value problem $\xi(0)=0, \xi^{\prime}(t)=f_{n}(\bar{\gamma}(t), \xi(t))$, such that

$$
\|\xi-\eta\|_{\infty} \leqslant \frac{\alpha_{n}}{k_{n}}\left(e^{k_{n}}-1\right) \leqslant 2^{-n-1} \varepsilon_{n} .
$$

Hence, $\operatorname{im}(\xi) \subseteq \Delta_{1-2^{-n-1}}^{d_{n}}$ in particular.

We now define $\Omega:=\Delta_{s_{1}}^{d_{n}} \oplus \bigoplus_{n \geqslant 2} \Delta_{s_{n}}^{d_{n}-d_{n-1}}$, considering $\mathbb{K}^{s}$ as the locally convex direct sum $\mathbb{K}^{d_{1}} \oplus \bigoplus_{n \geqslant 2} \mathbb{K}^{d_{n}-d_{n-1}}$. Then $\gamma_{0}+C^{1}([0,1], \Omega)$ is an open neighbourhood of $\gamma_{0}$ in $C^{1}([0,1], L(G))$. Let $\gamma \in \gamma_{0}+C^{1}([0,1], \Omega)$. Then $\gamma-\gamma_{0}=\sum_{n=1}^{\infty} \gamma_{n}$, where $\gamma_{n}$ is the coordinate function taking its values in $\Delta_{s_{1}}^{d_{1}}$, respectively in $\Delta_{s_{n}}^{d_{n}-d_{n-1}}$. There exists $\ell \in \mathbb{N}$ such that $\gamma_{n}=0$ for all $n \geqslant \ell$. Considering $\gamma_{0}, \gamma_{0}+\gamma_{1}, \ldots, \sum_{n=0}^{\ell} \gamma_{n}=\gamma$ in turn, from the existence of $\zeta_{0}$ we inductively deduce by the preceding arguments that there exists a solution $\zeta_{n}:[0,1] \rightarrow \Delta_{1-2^{-n-1}}^{d_{n}}$ to the initial value problem $\zeta_{n}^{\prime}(t)=f_{n}\left(\gamma_{0}(t)+\cdots+\gamma_{n}(t), \zeta_{n}(t)\right), \zeta_{n}(0)=0$, for $n=1, \ldots, \ell$, such that $\left\|\zeta_{n}-\zeta_{n-1}\right\|_{\infty} \leqslant$ $2^{-n-1} \varepsilon_{n}$ (see (4)). Then $\eta:=\phi \circ \zeta_{\ell}$ is the right product integral for $\gamma$, and thus $\operatorname{evol}_{G}^{r}(\gamma)=\eta(1) \in W$ because

$$
\phi^{-1}(\eta(1))-\phi^{-1}\left(\eta_{0}(1)\right)=\zeta_{\ell}(1)-\zeta_{0}(1)=\sum_{n=1}^{\ell}\left(\zeta_{n}(1)-\zeta_{n-1}(1)\right) \in \Delta_{\varepsilon_{1}}^{d_{1}} \oplus \bigoplus_{n=2}^{\ell} \Delta_{\varepsilon_{n}}^{d_{n}-d_{n-1}} \subseteq \phi^{-1}(W) .
$$

Hence, $\operatorname{evol}_{G}^{r}$ is indeed continuous at $\gamma_{0}$.

Step 5: $\operatorname{evol}_{G}^{r}$ is continuous. Let $\bar{\gamma} \in C^{1}([0,1], L(G))$. After passing to a subsequence, we may assume that $\operatorname{im}(\bar{\gamma}) \subseteq \mathbb{K}^{d_{1}}=L\left(G_{1}\right)$. Let $\bar{\eta}:=\operatorname{Evol}_{G}^{r}(\bar{\gamma})$. We find a partition $0=t_{0}<t_{1}<\cdots<t_{\ell}=1$ such that $\bar{\eta}_{j}([0,1]) \subseteq \phi_{1}\left(\Delta_{1 / 2}^{d_{1}}\right)$ for each $j \in\{0, \ldots, \ell-1\}$, where $\bar{\eta}_{j}:[0,1] \rightarrow G$ is defined via $\bar{\eta}_{j}(t):=\bar{\eta}\left(t_{j}+t\left(t_{j+1}-t_{j}\right)\right) \bar{\eta}\left(t_{j}\right)^{-1}$. Then $\bar{\eta}_{j}=\operatorname{Evol}_{G}^{r}\left(\bar{\gamma}_{j}\right)$, where $\bar{\gamma}_{j}:[0,1] \rightarrow L(G), \bar{\gamma}_{j}(t):=$ $\left(t_{j+1}-t_{j}\right) \cdot \bar{\gamma}\left(t_{j}+t\left(t_{j+1}-t_{j}\right)\right)$ are maps which satisfy the hypotheses of Step 4. Thus, evol ${ }_{G}^{r}$ is continuous at $\bar{\gamma}_{j}$. Since $\gamma \mapsto \gamma_{j}$ is continuous and $\operatorname{evol}_{G}^{r}(\gamma)=\operatorname{evol}_{G}^{r}\left(\gamma_{\ell-1}\right) \cdots \operatorname{evol}_{G}^{r}\left(\gamma_{1}\right) \operatorname{evol}_{G}^{r}\left(\gamma_{0}\right)$, we see that $\operatorname{evol}_{G}^{r}$ is continuous at $\bar{\gamma}$. (We have even established continuity with respect to the topology of uniform convergence!)

Step 6: $\operatorname{evol}_{G}^{r}$ is $C_{\mathbb{C}}^{\infty}$ if $\mathbb{K}=\mathbb{C}$. It suffices to show that $\operatorname{evol}_{G}^{r}$ is $C_{\mathbb{C}}^{\infty}$ on some open neighbourhood of each $\gamma_{0} \in C^{1}([0,1], L(G))$ such that $\gamma_{0}([0,1]) \subseteq L\left(G_{1}\right)$ and such that $\eta_{0}:=\operatorname{Evol}_{G}^{r}\left(\gamma_{0}\right)$ has image in $\phi_{1}\left(\Delta_{1 / 2}^{d_{1}}\right)$, by arguments similar to those just employed. Let $\Omega$ be as in Step 4 , and $U:=$ $\gamma_{0}+C^{1}([0,1], \Omega)$. As shown in Step $4, \eta:=\operatorname{Evol}_{G}^{r}(\gamma)$ has image in $Q=\operatorname{im}(\phi)$, for each $\gamma \in U$, $\zeta:=\phi^{-1} \circ \eta$ satisfies $\zeta(0)=0$, and $\zeta^{\prime}(t)=f_{n}(\gamma(t), \zeta(t))$ for each $n$ such that $\zeta([0,1]) \subseteq \mathbb{C}^{d_{n}}$. Now suppose that $\gamma \in U$ and $\theta \in C^{1}([0,1], L(G))$. There exists $n$ (which we fix now) such that $\gamma, \theta$ have image in $\mathbb{C}^{d_{n}}$. Then $\sigma_{z}:=\gamma+z \theta \in U$ for $z$ in some zero-neighbourhood $V \subseteq \mathbb{C}$, and $\operatorname{im}\left(\sigma_{z}\right) \subseteq \mathbb{C}^{d_{n}}$ for each $z \in V$. Let $\tau_{z}:=\phi^{-1} \circ \operatorname{Evol}_{G}^{r}\left(\sigma_{z}\right)$. Then $\tau_{z}$ solves the initial value problem $\tau_{z}(0)=0, \tau_{z}^{\prime}(t)=f_{n}\left(\sigma_{z}(t), \tau_{z}(t)\right)$. Consider $f:[0,1] \times \Delta_{1}^{d_{n}} \times V \rightarrow \mathbb{C}^{d_{n}}, f(t, x, z):=f_{n}\left(\sigma_{z}(t), x\right)$. Then $f(t, x, z)=f_{n}(\gamma(t), x)+z f_{n}(\theta(t), x)$, showing that the differentiability requirements of [Car71, Theorem 3.6.1] are satisfied. ${ }^{4}$ Hence, $u(t, z):=\tau_{z}(t)$ is $C_{\mathbb{R}}^{1}$ in $(t, z)$ on an open neighbourhood of $I \times\{0\}$ in $I \times V$, and the map $h:[0,1] \rightarrow \mathcal{L}_{\mathbb{R}}\left(\mathbb{C}, \mathbb{C}^{d_{n}}\right), h(t):=d_{2} u(t, 0, \bullet)$ to the space of $\mathbb{R}$-linear maps $\mathbb{C} \rightarrow \mathbb{C}^{d_{n}}$ is $C_{\mathbb{R}}^{1}$ and solves the initial value problem

$$
h(0)=0, \quad h^{\prime}(t)=b(t) \circ h(t)+c(t),
$$

\footnotetext{
${ }^{4}$ To apply the theorem, note that $f$ extends to an open set, because $\gamma$ and $\theta$ extend to open intervals by Borel's
} theorem. 


\section{H. GLÖCKNER}

where $c(t)(z)=z \cdot f_{n}\left(\theta(t), \tau_{0}(t)\right)$ and $b(t)=d_{2} f_{n}\left(\sigma_{0}(t), \tau_{0}(t), \bullet\right)$. Since $b(t) \in \mathcal{L}_{\mathbb{C}}\left(\mathbb{C}^{d_{n}}, \mathbb{C}^{d_{n}}\right)$ for each $t$ and $c(t) \in \mathcal{L}_{\mathbb{C}}\left(\mathbb{C}, \mathbb{C}^{d_{n}}\right)$, we can interpret $(5)$ also as a linear differential equation for $\mathcal{L}_{\mathbb{C}}\left(\mathbb{C}, \mathbb{C}^{d_{n}}\right)$ valued functions. This implies that $h(t) \in \mathcal{L}_{\mathbb{C}}\left(\mathbb{C}, \mathbb{C}^{d_{n}}\right)$ for each $t$, i.e., $h(t)=d_{2} u(t, 0, \bullet)$ is complex linear. Hence $d /\left.d z\right|_{z=0} \phi^{-1}\left(\operatorname{evol}_{G}^{r}(\gamma+z \theta)\right)=d /\left.d z\right|_{z=0} \tau_{z}(1)=\partial /\left.\partial z\right|_{z=0} u(1, z)$ exists as a complex derivative.

By the preceding, $\psi:=\left.\phi^{-1} \circ \operatorname{evol}_{G}^{r}\right|_{U}: U \rightarrow \mathbb{C}^{s}$ admits complex directional derivatives at each point. Hence, $\psi$ is G-analytic in the sense of [BS71, Definition 5.5], by [BS71, Proposition 5.5] and [BS71, Theorem 3.1]. Being G-analytic and continuous, $\psi$ is complex analytic [BS71, Theorem 6.1(i)].

Step 7: $\operatorname{evol}_{G}^{r}$ is $C_{\mathbb{R}}^{\infty}$ and $c_{\mathbb{R}}^{\omega}$ if $\mathbb{K}=\mathbb{R}$. Because $G$ is assumed simply connected, we know that $H:=\gamma_{G}(G)$ is a closed subgroup of $G_{\mathbb{C}}$, that $\gamma_{G}$ has discrete kernel, and that $\gamma_{G}$ is a local $c_{\mathbb{R}^{-}}^{\omega}$ diffeomorphism onto $H$, equipped with the real Lie group structure induced by $\left(G_{\mathbb{C}}\right)_{\mathbb{R}}$ (see Proposition 7.13(c) and (d)). Since $H$ is $C_{\mathbb{R}}^{\infty}$-initial in $G_{\mathbb{C}}$ and $c_{\mathbb{R}^{\omega}}^{\omega}$-initial (Proposition 7.5(b)), we deduce from the smoothness (and $c_{\mathbb{R}}^{\omega}$-property) of $\gamma_{G} \circ \operatorname{evol}_{G}^{r}=\operatorname{evol}_{G_{\mathbb{C}}}^{r} \circ L\left(\gamma_{G}\right)$ that $\left.\gamma_{G}\right|^{H} \circ \mathrm{evol}_{G}^{r}$ is $C_{\mathbb{R}}^{\infty}$ and $c_{\mathbb{R}}^{\omega}$. As evol ${ }_{G}^{r}$ is continuous and $\left.\gamma_{G}\right|^{H}$ a local $C_{\mathbb{R}}^{\infty}$ - (and $\left.c_{\mathbb{R}^{-}}^{\omega}\right)$ diffeomorphism, this implies that evol ${ }_{G}^{r}$ is $C_{\mathbb{R}}^{\infty}$ and $c_{\mathbb{R}}^{\omega}$.

\section{REFERENCES}

Anc76 V. Ancona, Sui fibrati analitici E-principali II: Teoremi di classificazione, Rend. Sem. Mat. Univ. Padova 55 (1976), 50-62.

BGN04 W. Bertram, H. Glöckner and K.-H. Neeb, Differential calculus over general base fields and rings, Expo. Math. 22 (2004), 213-282.

BS71 J. Bochnak and J. Siciak, Analytic functions in topological vector spaces, Studia Math. 39 (1971), $77-112$.

Bou89 N. Bourbaki, Lie Groups and Lie Algebras, Chapters 1-3 (Springer, Berlin, 1989).

Car71 H. Cartan, Differential Calculus (Hermann, Paris, 1971).

Die60 J. Dieudonné, Foundations of Modern Analysis (Academic Press, Reading, MA, 1960).

Eng89 R. Engelking, General Topology (Heldermann, Berlin, 1989).

Glo02a H. Glöckner, Lie group structures on quotient groups and universal complexifications for infinitedimensional Lie groups, J. Funct. Anal. 194 (2002), 347-409.

Glo02b H. Glöckner, Infinite-dimensional Lie groups without completeness restrictions, in Geometry and Analysis on Finite- and Infinite-Dimensional Lie Groups, eds A. Strasburger et al., Banach Center Publications, vol. 55 (Banach Center, Warsaw, 2002), 43-59.

Glo03a H. Glöckner, Direct limit Lie groups and manifolds, J. Math. Kyoto Univ. 43 (2003), 1-26.

Glo03b H. Glöckner, Implicit functions from topological vector spaces to Banach spaces, TU Darmstadt Preprint 2271 (2003), arXiv:math.GM/0303320.

Glo03c H. Glöckner, Every smooth p-adic Lie group admits a compatible analytic structure, Forum Math., to appear, arXiv:math.GR/0312113.

Glo04 H. Glöckner, Infinite-dimensional Lie groups over topological fields, cf. TU Darmstadt Preprint 2356 (2004), arXiv:math.GM/0408008.

Glo05 H. Glöckner, Smooth Lie groups over local fields of positive characteristic need not be analytic, J. Algebra 285 (2005), 356-371.

GN03 H. Glöckner and K.-H. Neeb, Banach-Lie quotients, enlargibility, and universal complexifications, J. reine angew. Math. 560 (2003), 1-28.

Gra58a H. Grauert, Analytische Faserungen holomorph-vollständiger Räume, Math. Ann. 135 (1958), 263-273.

Gra58b H. Grauert, On Levi's problem and the imbedding of real-analytic manifolds, Ann. of Math. (2) 68 (1958), 460-472. 


\section{FUndAMENTALS OF DIRECT Limit LiE THEORY}

Gua02 F. Guaraldo, On real analytic fibre bundles: classification theorems, Rev. Roumaine Math. Pures Appl. 47 (2002), 305-314.

Han71 V. L. Hansen, Some theorems on direct limits of expanding systems of manifolds, Math. Scand. 29 (1971), 5-36.

Hir76 M. W. Hirsch, Differential Topology (Springer, New York, 1976).

HM98 K. H. Hofmann and S. A. Morris, The Structure of Compact Groups (de Gruyter, Berlin, 1998).

HN91 J. Hilgert and K.-H. Neeb, Lie-Gruppen und Lie-Algebren (Vieweg, Braunschweig, 1991).

HST01 T. Hirai, H. Shimomura, N. Tatsuuma and E. Hirai, Inductive limits of topologies, their direct products, and problems related to algebraic structures, J. Math. Kyoto Univ. 41 (2001), 475-505.

KM97 A. Kriegl and P. W. Michor, The Convenient Setting of Global Analysis, Mathematical Surveys and Monographs, vol. 53 (American Mathematical Society, Providence, RI, 1997).

Lan99 S. Lang, Fundamentals of Differential Geometry (Springer, Berlin, 1999).

Mil82 J. Milnor, On infinite dimensional Lie groups, Preprint (1982), Institute for Advanced Study, Princeton, NJ.

Mil83 J. Milnor, Remarks on infinite dimensional Lie groups, in Relativity, Groups and Topology II, eds B. DeWitt and R. Stora (North-Holland, Amsterdam, 1983).

NRW91 L. Natarajan, E. Rodríguez-Carrington and J. A. Wolf, Differentiable structure for direct limit groups, Lett. Math. Phys. 23 (1991), 99-109.

NRW93 L. Natarajan, E. Rodríguez-Carrington and J. A. Wolf, Locally convex Lie groups, Nova J. Algebra Geom. 2 (1993), 59-87.

NRW01 L. Natarajan, E. Rodríguez-Carrington and J. A. Wolf, The Bott-Borel-Weil theorem for direct limit groups, Trans. Amer. Math. Soc. 353 (2001), 4583-4622.

Roy74 H. L. Royden, The extension of regular holomorphic maps, Proc. Amer. Math. Soc. 43 (1974), 306-310.

Ser92 J.-P. Serre, Lie Groups and Lie Algebras (Springer, Berlin, 1992).

TSH98 N. Tatsuuma, H. Shimomura and T. Hirai, On group topologies and unitary representations of inductive limits of topological groups and the case of the group of diffeomorphisms, J. Math. Kyoto Univ. 38 (1998), 551-578.

Tog67 A. Tognoli, Sulla classificazione dei fibrati analitici reali, Ann. Scuola Norm. Sup. Pisa Cl. Sci. 21 (1967), 709-744.

Wei67 A. Weil, Basic Number Theory (Springer, Berlin, 1967).

Wol04 J. A. Wolf, Principal series representations of direct limit groups, Compositio Math. 141 (2005), 1504-1530.

Helge Glöckner gloeckner@mathematik.tu-darmstadt.de

Department of Mathematics, AG 5, Darmstadt University of Technology, Schlossgartenstrasse 7, 64289 Darmstadt, Germany 